Western Australian Telecommunications Research Institute

(WATRI)

\title{
The Impact of Transmission Line Terminations on Radiated Emissions
}

Bert Wei-Juet Wong

This thesis is presented for the award of the Degree of Masters of the

Curtin University of Technology 


\section{Declaration}

To the best of my knowledge and belief this thesis contains no material previously published by any other person except where due acknowledgment has been made.

This thesis contains no material which has been accepted for the award of any other degree or diploma in any university.

Signature:

Date: $\quad .14 / 03 / 07$ 
To My Family 


\section{Acknowledgements}

I wish to express my sincere thanks to my supervisors, Prof Kevin Fynn and Prof Antonio Cantoni, for their guidance and assistance throughout my Masters degree. I consider myself very privileged to have had the opportunity to learn from them.

In particular, I would like to acknowledge Prof Kevin Fynn for his many helpful discussions and for arranging an industrial secondment at EMC Technologies, an accredited testing house in the area of electromagnetic compatibility. Thank you for your offers for me to be part of the EMC research group such as WACET and ACETT. In addition, I would like to express my gratitude for his recommendations to SpecTerra Systems, especially working on the scanner project, and many other employment opportunities within WATRI. Many thanks for the ongoing support in my postgraduate studies.

I am very thankful to have Prof Antonio Cantoni as my Associate Supervisor. His ongoing encouragement and patience in this research has allowed me to meet the EMC challenges. Thank you for the many useful discussions, invaluable teaching, reviews of this thesis and helpful advice.

Furthermore, I would also like to acknowledge the support of the ATRI, ATcrc, TEN Research Group and WATRI. Thanks to the staff and students for providing a stimulating and pleasant work environment.

Additionally, I would like to acknowledge the financial support provided by the Curtin University Postgraduate Scholarship, the ATcre Top-Up Scholarship and the WATRI Ad-Hoc Scholarship.

Thanks to Dr Franz Schlagenhaufer for his continued guidance and Mr Jian He with the CONCEPT simulations.

Special thanks to my family for their ongoing encouragement and support, especially my Mother.

Thanks also to my ex-room mates at ATRI, Mr Peter Wong and Mr Charles Voo for their continued friendship. 
Finally, I would like to give a big thank you to Dr Joe Trinkle for his constant involvement in assisting me overcome difficult hurdles. He is always encouraging and ready to help answer my questions in electromagnetics. 


\section{Abstract}

Terminating transmission lines at either the source or the load end with a damping resistor is frequently used to improve the signal integrity of clock or control lines on printed circuit boards. While a number of termination variants may produce similar results in regards to signal integrity, the electromagnetic compatibility performance can vary due to different current and voltage distributions along the line. This thesis investigates the impact of transmission line terminations on radiated emissions. 


\section{Table of Contents}

$\begin{array}{lr}\text { Author's Publications } & 1\end{array}$

$\begin{array}{lr}\text { Chapter 1. Introduction } & 2\end{array}$

1.1 Background 2

1.2 Motivation 3

1.3 Microstrip Model 4

1.4 Summary of Contributions 6

1.5 Thesis Outline $\quad 7$

$\begin{array}{ll}\text { Chapter 2. Current Distributions } & 9\end{array}$

2.1 Introduction 9

2.2 Distributed Transmission Line Current 10

2.2.1 Current Distributions 11

2.2.2 Current Derivative Distributions 12

2.2.3 Current Distribution Summary 13

2.2.4 Special Cases $\quad 13$

2.2.5 Source and Load Currents 14

2.3 Polarisation Currents 15

2.3.1 Dielectric Polarisation Current Model 16

2.3.2 Simple Dielectric Polarisation Current Derivation 17

2.3.3 Computing Transmission Line Polarisation Currents 18

2.3.4 Computing Polarisation Current Dipoles 19

2.4 Microstrip Characterisation $\quad 20$

$\begin{array}{ll}\text { 2.4.1 Wheeler's Microstrip Equations } & 20\end{array}$

2.4.2 Effective Transmission Line Characteristics $\quad 21$

2.5 Conclusion $\quad 23$

$\begin{array}{lr}\text { Chapter 3. Simple Radiation Models } & 24\end{array}$

3.1 Introduction 24

3.2 Electric Field from Currents $\quad 25$

3.3 Hertzian Dipole Model

$\begin{array}{ll}3.4 \text { Schelkunoff Model } & 28\end{array}$

3.5 Travelling Line Current 30

3.6 Closed Loop Transmission Line Radiation 34

3.7 Current Loop with Dielectric

3.8 Microstrip with Arbitrary Termination 42

3.9 Conclusion 44 
4.1 Introduction $\quad 45$

4.2 Test Facility $\quad 45$

4.3 Test Measurement Setup $\quad 45$

4.4 Measurement Procedure 46

4.4.1 Ground Plane Improvements $\quad 47$

4.4.2 Loop and Antenna Calibration $\quad 48$

4.5 Measurement Results 49

$\begin{array}{ll}\text { 4.5.1 Matched Load } & 50\end{array}$

$\begin{array}{ll}\text { 4.5.2 Short } & 51\end{array}$

4.5.3 Open $\quad 52$

4.5.4 $22 \mathrm{pF}$ Capacitor $\quad 53$

4.6 Conclusion $\quad 54$

$\begin{array}{ll}\text { Chapter 5. Source and Destination Damping of TLs } & 56\end{array}$

5.1 Introduction $\quad 56$

5.2 Damping

5.3 Load Voltage $\quad 60$

5.4 Results

5.5 Conclusion $\quad 65$

$\begin{array}{lr}\text { Chapter 6. Conclusions and Future Work } & 66\end{array}$

$\begin{array}{lr}\text { Appendix A. Results for Chapter 2 } & 67\end{array}$

A.1 Transmission Line Wave Equations

A.2 Voltage and Current along a Transmission Line 71

A.3 Voltage and Current along a Lossless Transmission Line 75

$\begin{array}{ll}\text { A.3.1 Special Cases } & 76\end{array}$

$\begin{array}{ll}\text { A.3.1.1 Short Circuit Load } & 77\end{array}$

$\begin{array}{ll}\text { A.3.1.2 Open Circuit Load } & 77\end{array}$

$\begin{array}{ll}\text { A.3.1.3 Boundary Ends } & 78\end{array}$

$\begin{array}{ll}\text { A.3.1.3.1 Short Circuit Load } & 79\end{array}$

$\begin{array}{ll}\text { A.3.1.3.2 Open Circuit Load } & 80\end{array}$

A.4 Decibel to Neper Conversion

$\begin{array}{lr}\text { Appendix B. Results for Chapter } 3 & 82\end{array}$

B.1 Schelkunoff's Equations in Compact Form

B.2 Electric Field of a Hertzian Dipole Arbitrarily Placed in free Space 85

B.1.1 Electric Field in Cartesian Coordinates $\quad 87$

B.1.2 Near-Zone Far-Zone Boundary $\quad 89$ 
Appendix C. Glossary

C.1 Mathematical Conventions 90

C.2 Abbreviated Units 90

C.3 Table of Definitions

$\begin{array}{ll}\text { C.4 Physical Constants } & 93\end{array}$

$\begin{array}{ll}\text { C.5 Acronyms } & 93\end{array}$

$\begin{array}{lr}\text { Appendix D. Chamber Map } & 94\end{array}$

References

95 


\section{Author's Publications}

1. Wong, B, Fynn, K, Schlagenhaufer, F \& Cantoni, A 2001, 'The impact of transmission line terminations on radiated emissions', IEEE International Symposium on EMC, vol.1, pp. 52-56.

2. Wong, B, Cantoni, A, Fynn, K \& Trinkle, J 2005, 'Effect of modelling fringing and losses for a microstrip on the radiated emission characteristics', IEEE International Symposium on EMC, pp. 557-562.

3. Wong, B \& Trinkle, J 2006, 'Accurate measurements in semi-anechoic chambers', Electromagnetic Compatibility Symposium, Electromagnetic Compatibility Society of Australia, IEEE Electromagnetic Compatibility Society, pp. 11-20. 


\section{Chapter 1. Introduction}

\subsection{Background}

All electrical and electronic products exhibit some form of unintended emission. It is because of this concern that many governing bodies set up electromagnetic compliance standards. Most industrialised countries have regulatory agencies for setting and monitoring electromagnetic compatibility (EMC) requirements. In Australia, the Australian Communications Authority (ACA) plays this role, whereas in other parts of the world, many countries follow the Federal Communications Commissions (FCC) or the European Economic Community (EEC). From an EMC standpoint a product is said be electromagnetic compatible when it functions satisfactorily in the intended electromagnetic environment without introducing intolerable electromagnetic disturbances to anything in that environment.

Currently many manufacturers are adopting EMC strategies which address the complete product development cycle. The traditional approach has been to perform compliance testing on the prototype unit and hope that it passes but the risks of this approach are becoming unacceptable. Increasingly strict regulations and growing product complexity make it difficult for manufacturers to pass the first time round.

Addressing EMC at an early stage in the product development cycle is challenging but often more cost effective in the long term. The early stages of a design allow designers the most freedom to achieve EMC. For example, a radiated emission problem may be addressed prior to building the prototype by applying certain design guidelines in the printed circuit board (PCB) layout.

Emissions are characterised as conducted or radiated. Conducted emissions come about when unwanted interference signals are conducted along cables. Radiated emissions, on the other hand, are coupled through electromagnetic fields by means of another medium, which usually is air.

The objective of the work reported in the thesis was to investigate the impact of transmission line (TL) terminations on radiated emission. In the process of 
experimentally validating the theoretical models developed, a methodology to obtain reliable radiated emission measurements for low radiating structures such as a trace was developed.

Source damping and destination damping are two widely used termination techniques available to the designer of high speed digital circuits to achieve signal integrity on digital interconnects. Both techniques provide a measure of loss to the TL that helps to dampen oscillating behaviour arising from the finite electrical length of the line. Source damping is achieved by adding a series resistance at the source end, i.e. in the forward path of the signal. In this scenario less power is launched into the TL, but once launched the majority of the signal will reflect at the load end and traverse the length of the line twice before being terminated at the source. For destination damping, a shunt resistance is added to the receiver, so that more signal is launched onto the TL, but very little is reflected at the load end. While, from a signal integrity point of view, both of these techniques can achieve the desired damping effect, they exhibit different current distributions on the trace. As a consequence, it is conceivable that their far field radiation patterns may be substantially different. As a result, from an EMC perspective, one method may be preferred over the other.

It is the objective of this thesis to identify the associated current distributions and to predict, by means of models, the electric field emissions if one technique is better than the other.

\subsection{Motivation}

With increasing clock frequencies, the electromagnetic radiation from traces on PCBs becomes an important consideration for compliance with EMC standards. Traces, in many cases are no longer electrically "short" and must be treated as transmission lines. The trace terminations will not only have an impact on signal integrity issues but also on radiation performance, so it is important to develop models for radiated emissions from PCB traces and to evaluate termination strategies from an EMC standpoint. 


\subsection{Microstrip Model}

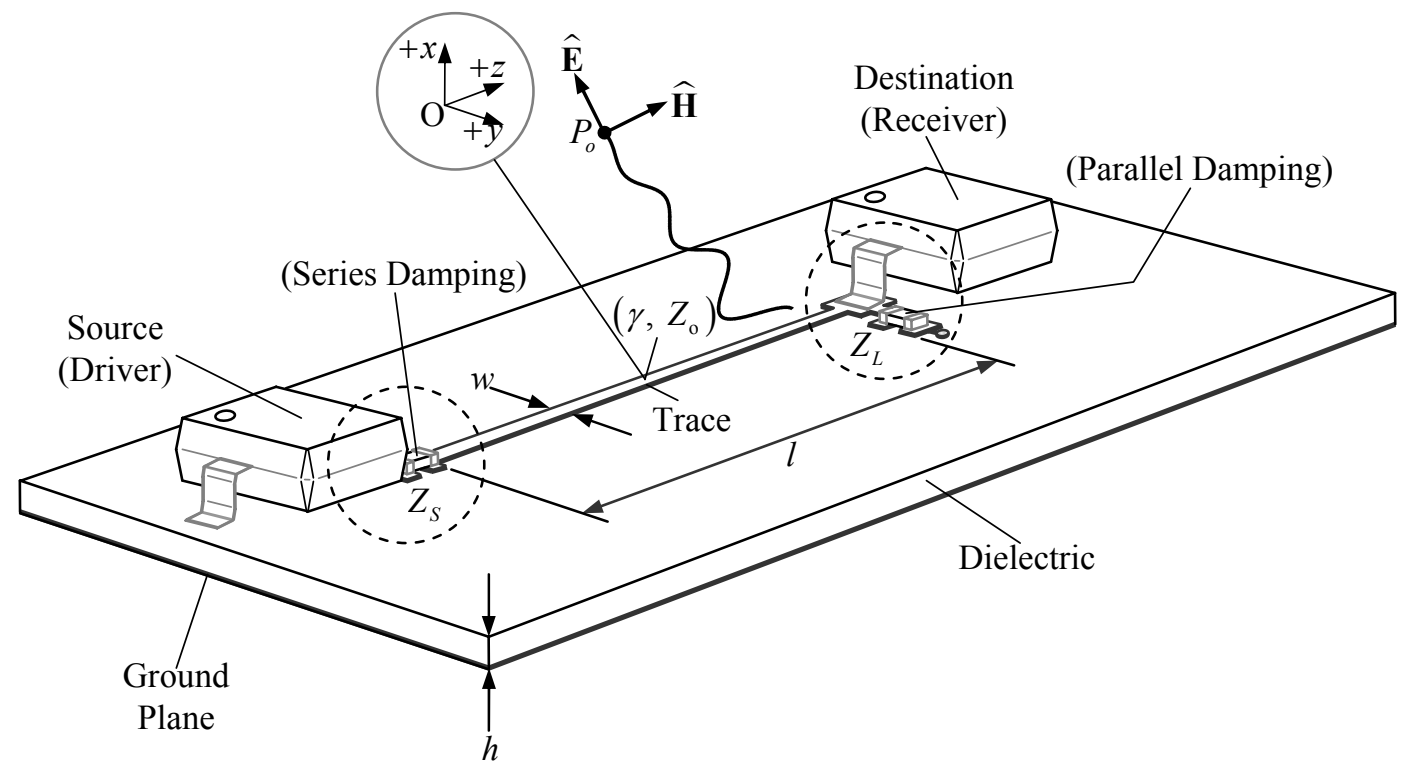

Figure 1.1 Problem definition.

Consider a microstrip with a driver and a receiver at the ends of the trace. The trace is characterised by its propagation constant, $\gamma(f, w, h)$, and characteristic impedance, $Z_{\mathrm{o}}(f, w, h)$, both of which are a function of frequency, $f$, width, $w$, and height, $h$, ignoring the trace thickness. Figure 1.1, illustrates the two termination definitions of source damping or destination damping as will be investigated in this thesis. They are represented as series and parallel damping resistors, respectively.

From TL theory currents anywhere along the trace of length, $l$, can be computed from its characteristic $\left(\gamma, Z_{\mathrm{o}}\right)$ together with a known source impedance, $Z_{S}$, and load, $Z_{L}$. These represent equivalent impedances looking out of the TL, treated as a two port network, at the ends. As indicated in Figure 1.1, the dotted circles define the location of the driver and receiving end whereby, $Z_{S}$ consists of a series damping and the driver's output impedance, while $Z_{L}$, is a parallel combination of the load damping and the input gate capacitance of the receiver. 
Once the current distribution is known the fields, can be computed at a desired observation point $P_{o}$ with respect to an origin $\mathrm{O}$, for a range of frequencies. This thesis gives the progressive development of models to predict the electric fields $\widehat{\mathbf{E}}$ for a typical loaded microstrip structure. These models are verified by measurements and are used to compare source and destination damping from an EMC perspective.

A number of assumptions and approximations are made in the development of a model for the physical microstrip structure so that electric field emissions can be obtained. These fall into two groups. The first group includes the assumptions required to transform the physical microstrip into a current distribution in free space. The second group includes the approximations required to determine the electric field from the free space current distribution using Hertzian dipoles. The relevant assumptions are summarised below:

Physical Microstrip $\rightarrow$ Current Distribution in Free Space

AS1. For the purposes of determining the current distribution, only copper and dielectric loses will be considered. It is assumed that radiation losses are negligible compared to these. This assumption is required to make the mathematical analysis analytically tractable. This is because radiation loses are a complicated function of frequency and the microstrip's geometrical parameters $[22,30]$. It will be demonstrated later in this thesis that this assumption is valid for the typical trace considered in this.

AS2. Quasi-TEM propagation along the microstrip up to $1 \mathrm{GHz}$ for typical dimensions (i.e. for a $30 \mathrm{~cm}$ long, $3 \mathrm{~mm}$ wide and $1.5 \mathrm{~mm}$ high trace). This assumption allows the use of TL theory to determine the current distribution on the trace. Further, it is assumed that formulas given in well recognised textbooks such as [15] can be used to determine the per unit length parameters.

AS3. The physical dielectric can be removed and its effect modelled in free space by a sheet of polarisation current flowing vertically between the TL conductors, as suggested in [9]. 
AS4. The source impedance and load impedance can be treated as lumped LCR circuits.

AS5. The ground plane is perfectly conducting and of infinite extent so that image theory can be applied to replace the physical ground plane with an image current distribution in free space.

Current Distribution in Free Space $\rightarrow$ Electric Field Emissions

AP1. The trace is sufficiently narrow and long so that the surface current flowing on the trace due to skin effect can be treated as a line filament of current. This allows the line currents to be discretised into many Hertzian dipoles [15], and their contributions to be added up to give the total electric field due to the line currents.

AP2. The TL conductors are close together so that the polarisation current distribution can be discretised into vertical Hertzian dipoles in the cross section and their contributions added up to give the total field due to polarisation.

\subsection{Summary of Contributions}

The key contributions of this thesis are:

I. The conclusion that for realistic values of damping there is little difference between source and destination damping from an emissions point of view.

II. The development of a computational method for determining electric fields from a simple microstrip utilising Hertzian dipoles based on the physical structure of the microstrip.

III. The identification of the key factors required to predict the electric fields generated by a microstrip.

IV. The development of a measurement methodology to obtain reliable and repeatable measurement results for validating the analytical model. 
V. The work highlights the limitations of Schelkunoff's analytic equations when used to model the electric field generated by a microstrip.

\subsection{Thesis Outline}

The thesis structure is shown diagrammatically illustrated in Figure 1.2.

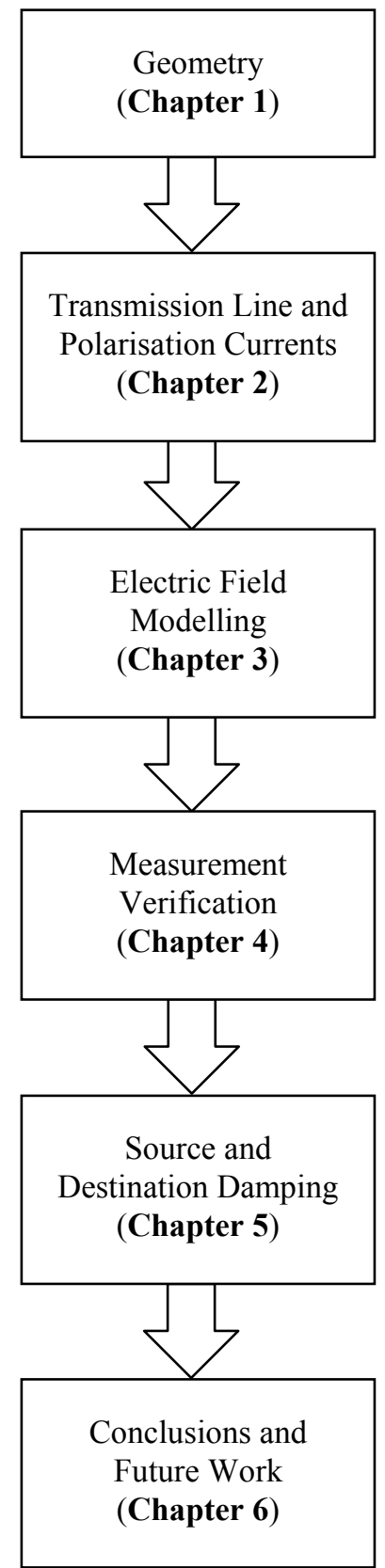

Figure 1.2 Thesis outline. 
Chapter 1 contains information that addresses certain issues in EMC. Chapter 2 presents a summary of TL current distributions used in modelling the differential currents on the microstrip. Polarisation currents are also given, which have a significant role in the modelling of the microstrip's radiation. Wheeler's equations [15] are introduced to obtain a characterisation of the microstrip in terms of the characteristic impedance and propagation constant.

Chapter 3 uses the current distribution obtained in Chapter 2 to obtain the electric field. Two electric field modelling methods are presented: (i) Hertzian and (ii) Schelkunoff. Comparisons are made using both methods discussing the advantages and disadvantages of each from the point of view of modelling radiated emissions from the microstrip. At the beginning comparisons are made in free space before including the dielectric of the microstrip.

Verification of the electric field modelling is performed with measurements. The measurements obtained confirmed the appropriateness of the model by measurement is given in Chapter 4. This chapter can be considered as a laboratory report on the setup so that good repeatable measurement results may be obtained.

Chapter 5 ties together the results from the previous chapters. It contains results addressing the impact of source series and destination parallel terminations on the radiated emissions.

Conclusions regarding the field emissions from a typical PCB trace under the two different damping schemes are presented in Chapter 6 and future work is identified. 


\section{Chapter 2. Current Distributions}

\subsection{Introduction}

In order to predict radiated fields from the microstrip line, it is first necessary to model the current distributions on the entire structure. Each element of current, be it on the trace, on the ground plane, or associated with the dielectric polarisation, will contribute to the overall radiation. Accurate determination of current distributions is a difficult task and may only be achieved with full wave modelling. Some full wave modelling techniques available are FDTD, MoM and PEEC [19, 24, 25, 28]. As these numerical methods require the meshing of the geometry, and for some the entire domain, these solutions can be very computationally expensive. However, under the assumption that the current distribution is not affected by power loss due to radiation, the task of finding the current distribution is greatly simplified and TL theory may be used [17].

In many instances common-mode currents on PCB structures, which are difficult to identify, are the dominating sources, $[5,29]$. The scope of this thesis is restricted to TL currents as field sources. Future research on the radiation due to common-mode currents is required. Such analysis is particularly important in the study of parallel traces used for differential signalling or in the study of radiation from twisted pair or ribbon cabling.

This chapter has three main sections. In the first section TL current distribution is presented. The second section presents currents due to dielectric polarisation. The last section covers methods required to readily compute the parameters $\left(Z_{\mathrm{o}}, \gamma\right)$ that characterise the microstrip. 
The following key topics are covered:

1. The generalised current distribution on a TL from classical TL theory and its associated limitations.

2. The boundary currents and corresponding derivatives that are necessary in the computation of the electric field based on Schelkunoff's derivations.

3. The source of polarisation currents that exists within the substrate for two parallel conductors.

4. Wheeler's equations for a microstrip to obtain the effective relative permittivity, characteristic impedance and the propagation constant of the TL including conduction and dielectric losses in the microstrip.

Derivations of the equations that arise in the first two key topics can be found in Appendix A.

\subsection{Distributed Transmission Line Current}

From classical TL theory, under the assumptions AS1 and AS2, currents directed along the $z$-axis given in Figure 2.1 satisfy the second order differential wave equation, or linear homogeneous DE, given by $[13,34]$

$$
\begin{gathered}
\frac{d^{2} \hat{I}(z)}{d z^{2}}=\gamma^{2} \hat{I}(z), \\
\gamma=\alpha+j \beta,
\end{gathered}
$$

where $\gamma$ is the propagation constant. Its real part, $\alpha$, is the attenuation constant in nepers per metre and the imaginary part, $\beta$, is the phase constant in radians per metre. This is also illustrated in Figure 2.1, with a TL driven by a source voltage, $\widehat{V}_{S}$, with a source impedance, $Z_{S}$ and loaded with $Z_{L}$. 




Figure 2.1 Loaded TL.

Equation (2.1.1) may have many solutions, but the current and voltage distributions along the TL become unique when some source and load impedance is introduced which satisfy the following set of boundary conditions:

$$
\begin{gathered}
\hat{I}\left(-\frac{l}{2}\right)=\frac{\hat{V}_{S}-\hat{V}\left(-\frac{l}{2}\right)}{Z_{S}} \\
\hat{I}\left(\frac{l}{2}\right)=\frac{\hat{V}\left(\frac{l}{2}\right)}{Z_{L}} .
\end{gathered}
$$

\subsubsection{Current Distributions}

The solution to equation (2.1.1), subject to the boundary conditions given by equations (2.2.1) and (2.2.2), describes the current distribution along a TL directed along the $z$-axis. The solution involves a forward and a backward propagating wave and can be written in terms of exponentials or hyperbolic functions as:

$$
\begin{gathered}
\hat{I}_{\text {lossy }}(z)=\frac{\hat{V}_{S}}{D_{\text {lossy }}}\left(Z_{\mathrm{o}} \cosh \gamma\left(\frac{l}{2}-z\right)+Z_{L} \sinh \gamma\left(\frac{l}{2}-z\right)\right), \\
D_{\text {lossy }}=Z_{\mathrm{o}}\left(Z_{S}+Z_{L}\right) \cosh \gamma l+\left(Z_{\mathrm{o}}^{2}+Z_{S} Z_{L}\right) \sinh \gamma l
\end{gathered}
$$

with the TL centred at the origin, $\mathrm{O}$. 
As can be seen from equations (2.3.1) and (2.3.2) currents along the line length $l$, depend on parameters such as the voltage excitation, $\hat{V}_{S}$, propagation constant $\gamma$, characteristic impedance, $Z_{\mathrm{o}}$, and the impedances, $Z_{S}$, and, $Z_{L}$.

For a lossless TL, $\alpha=0$ and hence equations (2.3.1) and (2.3.2) become

$$
\begin{gathered}
\hat{I}_{\text {lossless }}(z)=\frac{\hat{V}_{S}}{D_{\text {lossless }}}\left(Z_{\mathrm{o}} \cos \beta\left(\frac{l}{2}-z\right)+j Z_{L} \sin \beta\left(\frac{l}{2}-z\right)\right), \\
D_{\text {lossless }}=Z_{\mathrm{o}}\left(Z_{S}+Z_{L}\right) \cos \beta l+j\left(Z_{\mathrm{o}}^{2}+Z_{S} Z_{L}\right) \sin \beta l .
\end{gathered}
$$

The phase constant $\beta$ is given by

$$
\beta=\frac{\omega}{u}=\frac{2 \pi f}{u}
$$

where $u$ is the speed of propagation along the line.

A simple TL model can always be characterised by its propagation constant and characteristic impedance. These parameters are related to the cross-sectional dimensions of the TL structure and its constituent material parameters. When a voltage source excites the TL and drives a load, the current at location $z$ can be calculated via equations (2.3.1) and (2.4.1), for a lossy and lossless line respectively.

\subsubsection{Current Derivative Distributions}

As will be discussed in Chapter 3, a computationally efficient method for obtaining the electric field for a finite length of line current with a sinusoidal distribution is Schelkunoff's [13]. Schelkunoff showed that it is possible to derive analytical expressions relating the electric fields to the current distribution and top the first derivative of the current distribution evaluated at the ends of the line. It is stated in [13] that the current distribution satisfies the second order differential equation according to,

$$
\frac{d^{2} \hat{I}(z)}{d z^{2}}=\beta^{2} \hat{I}(z)
$$


where $\beta=\beta_{0}$, the wave number in free space. The derivative of the current distribution obtained by taking the first derivative of equations (2.4.1) is:

$$
{\widehat{I^{\prime}}}_{\text {lossless }}(z)=\frac{\hat{V}_{S} \beta}{D_{\text {lossless }}}\left(Z_{\mathrm{o}} \sin \beta\left(\frac{l}{2}-z\right)-j Z_{L} \cos \beta\left(\frac{l}{2}-z\right)\right) .
$$

The denominator $D_{\text {lossless }}$ remains the same as in equation (2.4.2). This derivative of the current distribution will be used in Chapter 3 for calculation of emissions using Schelkunoff's derivations.

\subsubsection{Current Distribution Summary}

\begin{tabular}{|c|c|c|}
\cline { 2 - 3 } \multicolumn{1}{c|}{} & Lossy & Lossless \\
\hline \hline$\hat{\boldsymbol{I}}(z)$ & $\frac{\hat{V}_{S}}{D}\left(Z_{\mathrm{o}} \cosh \gamma\left(\frac{l}{2}-z\right)+Z_{L} \sinh \gamma\left(\frac{l}{2}-z\right)\right)$ & $\frac{\hat{V}_{S}}{D}\left(Z_{\mathrm{o}} \cos \beta\left(\frac{l}{2}-z\right)+j Z_{L} \sin \beta\left(\frac{l}{2}-z\right)\right)$ \\
\hline$\hat{\boldsymbol{I}}^{\prime}(z)$ & Not Applicable & $\frac{\hat{V}_{S} \beta}{D}\left(Z_{\mathrm{o}} \sin \beta\left(\frac{l}{2}-z\right)-j Z_{L} \cos \beta\left(\frac{l}{2}-z\right)\right)$ \\
\hline $\boldsymbol{D}$ & $Z_{\mathrm{o}}\left(Z_{S}+Z_{L}\right) \cosh \gamma l+\left(Z_{\mathrm{o}}^{2}+Z_{S} Z_{L}\right) \sinh \gamma l$ & $Z_{\mathrm{o}}\left(Z_{S}+Z_{L}\right) \cos \beta l+j\left(Z_{\mathrm{o}}^{2}+Z_{S} Z_{L}\right) \sin \beta l$ \\
\hline
\end{tabular}

Table 2.1 Summary of current distributions.

This section is a tabled summary, as in Table 2.1, of the useful current distributions presented in sections 2.2.1 and 2.2.2.

\subsubsection{Special Cases}

For the purposes of validation, when comparing different electric field computation models, it is useful to examine a short circuit $\left(Z_{L}=0\right)$ and an open circuit $\left(Z_{L}=\infty\right)$ conditions as these represent extreme cases for $Z_{L}$. At low frequencies these load cases can easily be implemented in the measurement test setup. 
A summary of the current distributions for these conditions is given in Table 2.2 and in Table 2.3.

\begin{tabular}{|c|c|c|}
\cline { 2 - 3 } \multicolumn{1}{c|}{} & \multicolumn{2}{|c|}{ Short Circuit $\left(Z_{L}=\mathbf{0}\right)$} \\
\cline { 2 - 3 } & Lossy & Lossless \\
\hline \hline$\hat{\boldsymbol{I}}(z)$ & $\frac{\hat{V}_{S}}{D} \cosh \gamma\left(\frac{l}{2}-z\right)$ & $\frac{\hat{V}_{S}}{D} \cos \beta\left(\frac{l}{2}-z\right)$ \\
\hline$\hat{\boldsymbol{I}}^{\prime}(z)$ & Not Applicable & $\frac{\hat{V}_{S} \beta}{D} \sin \beta\left(\frac{l}{2}-z\right)$ \\
\hline $\boldsymbol{D}$ & $Z_{S} \cosh \gamma l+Z_{\mathrm{o}} \sinh \gamma l$ & $Z_{S} \cos \beta l+j Z_{\mathrm{o}} \sin \beta l$ \\
\hline
\end{tabular}

Table 2.2 Summary of current distributions for $Z_{L}=0$.

\begin{tabular}{|c|c|c|}
\cline { 2 - 3 } \multicolumn{1}{c|}{} & \multicolumn{2}{|c|}{ Open Circuit $\left(Z_{L}=\infty\right)$} \\
\cline { 2 - 3 } \multicolumn{1}{c|}{ Lossy } & Lossless \\
\hline \hline$\hat{\boldsymbol{I}}(z)$ & $\frac{\hat{V}_{S}}{D} \sinh \gamma\left(\frac{l}{2}-z\right)$ & $j \frac{\hat{V}_{S}}{D} \sin \beta\left(\frac{l}{2}-z\right)$ \\
\hline$\hat{\boldsymbol{I}}^{\prime}(z)$ & Not Applicable & $-j \frac{\hat{V}_{S} \beta}{D} \cos \beta(l-z)$ \\
\hline $\boldsymbol{D}$ & $Z_{\mathrm{o}} \cosh \gamma l+Z_{S} \sinh \gamma l$ & $Z_{\mathrm{o}} \cos \beta l+j Z_{S} \sin \beta l$ \\
\hline
\end{tabular}

Table 2.3 Summary of current distributions for $Z_{L}=\infty$.

\subsubsection{Source and Load Currents}

The currents at the source and load end of the TL are obtained by setting $z=-l / 2$ for the generator end and $z=l / 2$ for the load end. The value of the currents at the ends of the TL will be used in Chapter 3 for Schelkunoff's method. These source and load currents are derived in Appendix A, and are summarised in Table 2.4. 


\begin{tabular}{|c|c|c|c|}
\hline & General & $Z_{L}=\mathbf{0}$ & $Z_{L}=\infty$ \\
\hline$\hat{I}\left(-\frac{l}{2}\right)$ & $\frac{\widehat{V}_{S}}{D}\left(Z_{\mathrm{o}} \cos \beta l+j Z_{L} \sin \beta l\right)$ & $\frac{\hat{V}_{S}}{D} \cos \beta l$ & $j \frac{\hat{V}_{S}}{D} \sin \beta l$ \\
\hline$\hat{I}^{\prime}\left(-\frac{l}{2}\right)$ & $\frac{\hat{V}_{S} \beta}{D}\left(Z_{\mathrm{o}} \sin \beta l-j Z_{L} \cos \beta l\right)$ & $\frac{\hat{V}_{S} \beta}{D} \sin \beta l$ & $-j \frac{\hat{V}_{S} \beta}{D} \cos \beta l$ \\
\hline$\hat{\boldsymbol{I}}\left(\frac{l}{2}\right)$ & $\frac{\hat{V}_{S} Z_{\mathrm{o}}}{D}$ & $\frac{\hat{V}_{S}}{D}$ & 0 \\
\hline$\hat{\boldsymbol{I}}^{\prime}\left(\frac{\boldsymbol{l}}{2}\right)$ & $-j \frac{\hat{V}_{S} Z_{L} \beta}{D}$ & 0 & $-j \frac{\hat{V}_{S} \beta}{D}$ \\
\hline$D$ & $Z_{\mathrm{o}}\left(Z_{S}+Z_{L}\right) \cos \beta l+j\left(Z_{\mathrm{o}}^{2}+Z_{S} Z_{L}\right) \sin \beta l$ & $Z_{S} \cos \beta l+j Z_{\mathrm{o}} \sin \beta l$ & $Z_{\mathrm{o}} \cos \beta l+j Z_{S} \sin \beta l$ \\
\hline
\end{tabular}

Table 2.4 Summary of the bounded current distributions.

\subsection{Polarisation Currents}

The source of polarisation in dielectrics, from atoms or molecules, exists under the influence of an electric field. Dipoles are subsequently formed and have their moments in the direction of the applied field. When the field oscillates or changes in direction the dipoles must reorientate themselves in a finite time and this results in what is known as a polarisation current, $\hat{I}_{p o l}$. This is as shown in Figure 2.2.

These currents are important to identify as they will contribute to the radiation of a microstrip that has a dielectric between the trace and ground. 


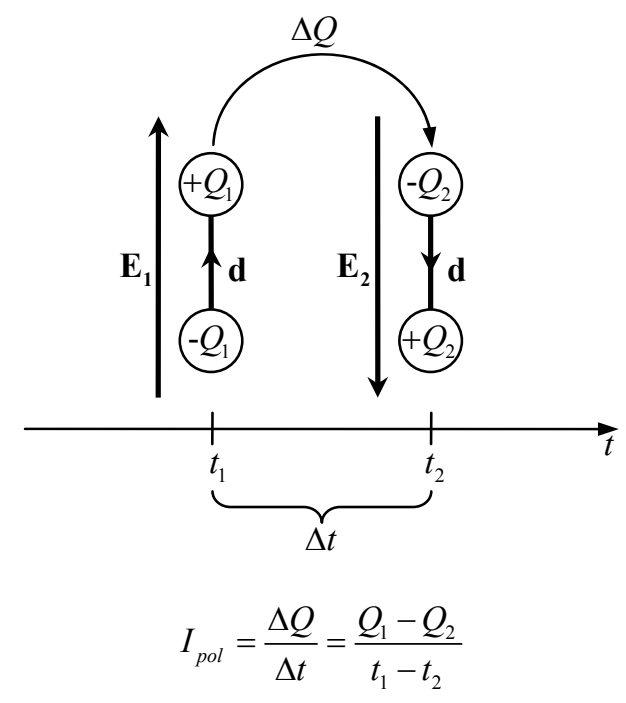

Figure 2.2 Polarisation current of a dipole moment in dielectrics.

\subsubsection{Dielectric Polarisation Current Model}

Consider a dielectric embedded between two parallel plates as shown in Figure 2.3.
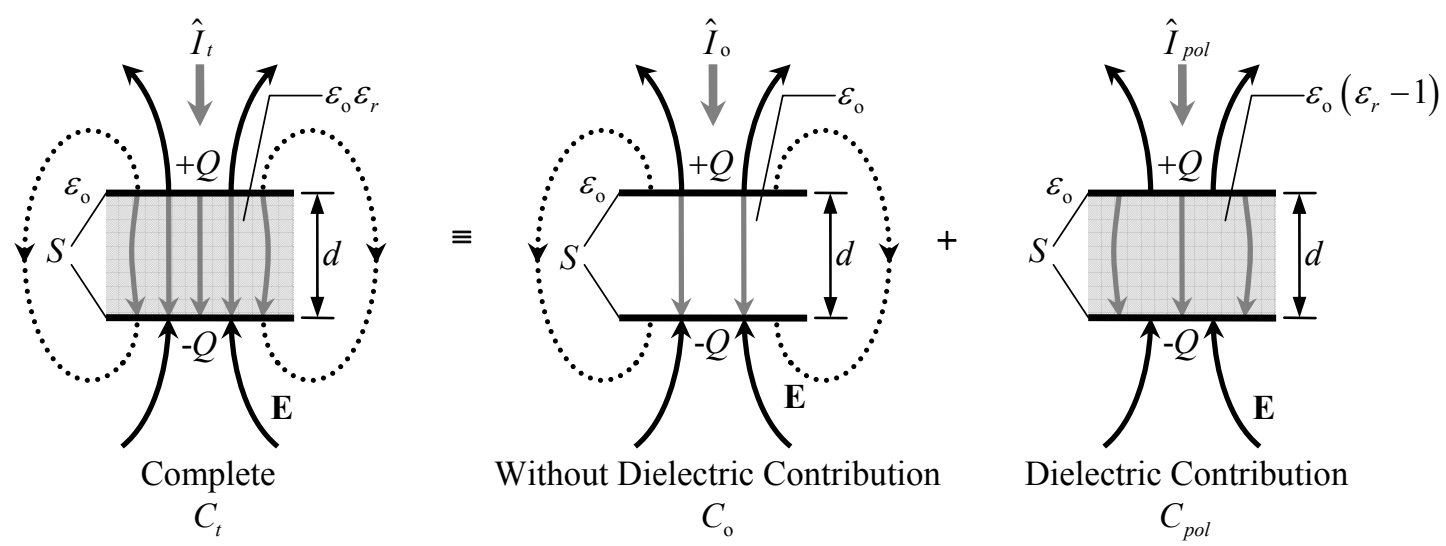

Figure 2.3 Decomposition of a parallel plate capacitor.

When the parallel plates carry charges, electric field lines will exist within the dielectric. This model decomposes the complete electric field from the capacitor as,

1. Contributions due to the conducting plates in free space;

2. Contribution due to polarisation of the dielectric medium. 


\subsubsection{Simple Dielectric Polarisation Current Derivation}

At low frequencies it is possible to isolate the electric field contributions, of the dielectric from the rest of the structure, as represented in Figure 2.3. This can be represented by the polarisation capacitance $C_{p o l}$. By assuming simple parallel plate capacitor behaviour with negligible fringing, total capacitance is given by

$$
C_{t}=\frac{\varepsilon_{0} \varepsilon_{r} S}{d}
$$

The capacitance, excluding the dielectric is

$$
C_{\mathrm{o}}=\frac{\varepsilon_{\mathrm{o}} S}{d}
$$

The polarisation current according to Figure 2.3 is given as

$$
\begin{aligned}
\hat{I}_{p o l} & =\hat{I}_{t}-\hat{I}_{\mathrm{o}} \\
& =j \omega\left(C_{t}-C_{\mathrm{o}}\right) \hat{V} \\
& =j \omega C_{t}\left(1-\frac{C_{\mathrm{o}}}{C_{t}}\right) \hat{V}, \\
& =j \omega C_{t}\left(1-\frac{1}{\varepsilon_{r}}\right) \hat{V}
\end{aligned}
$$

where $\hat{V}$ is the voltage across the capacitor.

Reference [9] provides a comprehensive proof of this result. As will be presented in Chapter 3, contributions to the electric field from the dielectric medium can be computed by treating them as Hertzian dipoles. 


\subsubsection{Computing Transmission Line Polarisation Currents}

A similar concept to that presented for the capacitor in the previous section can be applied to a TL to determine the polarisation currents. In a TL the per unit length polarisation current $\hat{J}_{p o l}(z)$ can be related to the per unit length capacitance $C$ and the voltage at that point $\widehat{V}(z)$ by equation (2.10) as follows:

$$
\hat{I}_{p o l}(z)=j \omega C_{t}\left(1-\frac{C_{\mathrm{o}}}{C_{t}}\right) \hat{V}(z)[\mathrm{A}] .
$$

Using equation A.19 given in Appendix A, it is possible to express $\widehat{J}_{\text {pol }}(z)$ in terms of the $d / d z$ of line currents as

$$
\widehat{J}_{p o l}(z)=-j \omega C\left(1-\frac{1}{\varepsilon_{r}}\right) \frac{1}{j \omega C+G} \frac{d \hat{I}(z)}{d z} .
$$

Assuming $G \simeq 0$ then

$$
\hat{J}_{p o l}(z)=-\left(1-\frac{1}{\varepsilon_{r}}\right) \frac{d \hat{I}(z)}{d z}\left[\frac{\mathrm{A}}{\mathrm{m}}\right] .
$$

In equation (2.10) a positive current is defined as flowing from the top positive plate to the negative bottom plate. In the TL model the sign is reversed as a current flowing from the top to the bottom line flows in the negative $x$ direction. Thus, removing the negative sign gives the polarisation current as

$$
\hat{\mathbf{J}}_{p o l}(z)=\left(1-\frac{1}{\varepsilon_{r}}\right) \frac{d \hat{I}(z)}{d z} \mathbf{a}_{x}\left[\frac{\mathrm{A}}{\mathrm{m}}\right] .
$$

Notice that if the dielectric is removed, $\varepsilon_{r}=1$ and $\widehat{J}_{p o l}(z)=0$ as expected. 


\subsubsection{Computing Polarisation Current Dipoles}

In Chapter 3, which models the electric field radiation, it is necessary to know polarisation currents of the dielectric in terms of dipoles.

Using

$$
\frac{\Delta \hat{I}(z)}{\Delta z} \approx \frac{\hat{I}\left(z+\frac{\Delta_{z}}{2}\right)-\hat{I}\left(z-\frac{\Delta_{z}}{2}\right)}{\Delta_{z}}
$$

in equation (2.14) and multiplying by $\Delta_{z}$ gives

$$
\hat{J}_{p o l}(z) \cdot \Delta_{z}=\left(\hat{I}\left(z+\frac{\Delta_{z}}{2}\right)-\hat{I}\left(z-\frac{\Delta_{z}}{2}\right)\right)\left(1-\frac{1}{\varepsilon_{r}}\right) .
$$

Consider now segmenting the line of length $l$ into $N$ segments of length $\Delta_{z}=l / N$. Further, assume $\hat{I}\left(z+\Delta_{z} / 2\right)$ is the current at the centre of the $n$th segment. If $\Delta=l / N, \hat{I}\left(z-\Delta_{z} / 2\right)$ is the current at the centre of segment $(n-1)$. Thus the polarisation current, $\hat{I}_{p o l_{n-1}}$ flowing between the lines at the join between segments $(n-1)$ and $n$ is $\hat{J}_{p o l}(z) \cdot \Delta_{z}$. Therefore

$$
\hat{I}_{p o l_{n-1}}=\left(\hat{I}_{n}-\hat{I}_{n-1}\right)\left(1-\frac{1}{\varepsilon_{r}}\right), \quad n \in[2, N],
$$

or

$$
\begin{aligned}
\hat{I}_{p o l_{n}} & =\left(\hat{I}_{n+1}-\hat{I}_{n}\right)\left(1-\frac{1}{\varepsilon_{r}}\right), \quad n \in[1, N-1] \\
& =\left(\hat{I}\left(-\frac{l}{2}+\left(n+\frac{1}{2}\right) \Delta_{z}\right)-\hat{I}\left(-\frac{l}{2}+\left(n-\frac{1}{2}\right) \Delta_{z}\right)\right)\left(1-\frac{1}{\varepsilon_{r}}\right), \quad n \in[1, N-1],
\end{aligned}
$$

where $\hat{I}_{p o l_{1}}$ for $n=1$ is the first polarisation current element closest to the source and is located at $z=-l / 2+\Delta_{z}$. 
In reality the polarisation currents do not flow as a line current in the cross-sectional plane of the TL but as a distributed current. However, since the cross-sectional dimension of the TL is small and the current distribution is symmetrical about the centre of the TL, the entire distribution can be represented to a good approximation by a string of dipoles at the centre of the line for radiation purposes.

\subsection{Microstrip Characterisation}

This section gives the parameters of the TL model for the microstrip defined in Chapter 1 in terms of the physical parameters of the microstrip. Empirical formulae, based on Wheeler's equations, are used for calculating the effective relative permittivity, characteristic impedance and propagation constant for the line.

\subsubsection{Wheeler's Microstrip Equations}

Given the microstrip problem defined in Chapter 1, and ignoring the strip conductor thickness, these definitions can be made [15]

$$
\begin{gathered}
A=\frac{120}{\sqrt{2\left(\varepsilon_{r}+1\right)}}, \\
B=\frac{1}{2}\left(\frac{\varepsilon_{r}-1}{\varepsilon_{r}+1}\right)\left(\ln \frac{\pi}{2}+\frac{1}{\varepsilon_{r}} \ln \frac{4}{\pi}\right), \\
C=\ln \frac{8 h}{w}+\frac{1}{32}\left(\frac{w}{h}\right)^{2}, \\
D=\frac{60 \pi}{\sqrt{\varepsilon_{r}}}, \\
+\frac{\varepsilon_{r}+1}{2 \pi \varepsilon_{r}}\left(1.452+\ln \left(\frac{w}{2 h}+0.94\right)\right)
\end{gathered}
$$


Equations (2.19.1), (2.19.2) and (2.19.4) depend on the relative permittivity, $\varepsilon_{r}$, equation (2.19.3) on the ratio $w / h$ and equation (2.19.5) on both. Parameters $A, B$, $C, D, E$ are used to compute the effective relative permittivity and characteristic impedance for narrow strip lines and wide strip lines. A summary is given in Table 2.5 .

\begin{tabular}{|c|c|c|}
\cline { 2 - 3 } \multicolumn{1}{c|}{} & Narrow Strip & Wide Strip \\
\hline \hline$\varepsilon_{\text {eff }}$ & $\varepsilon_{e f f} \approx \frac{\varepsilon_{r}+1}{2\left(1-\frac{B}{C}\right)^{2}},\left(\frac{w}{h}<1.3\right)$ & $\varepsilon_{e f f} \approx \frac{\varepsilon_{r}+1}{2}+\left(\frac{\varepsilon_{r}-1}{2}\right) \frac{1}{\sqrt{1+\frac{10 h}{w}}},\left(\frac{w}{h}>1.3\right)$ \\
\hline$Z_{\mathbf{0}}$ & $Z_{o} \approx A(C-B),\left(\frac{w}{h}<3.3\right)$ & $Z_{o} \approx \frac{D}{E},\left(\frac{w}{h}>3.3\right)$ \\
\hline
\end{tabular}

Table 2.5 Summary of $\varepsilon_{\text {eff }}$ and $Z_{\mathrm{o}}$.

\subsubsection{Effective Transmission Line Characteristics}

The equations given in Table 2.5 are good approximations, however better approximations are obtained by allowing these quantities to be frequency dependent $[26,27,31,34]$. From the newly calculated effective relative permittivity and characteristic impedance the phase velocity of a wave propagating along the microstrip is given by:

$$
u=\frac{c_{\mathrm{o}}}{\sqrt{\varepsilon_{\mathrm{eff}}}} .
$$

The propagation constant given by equation (2.1.2) may be rewritten as:

$$
\gamma(\omega)=\alpha(\omega)+j \beta(\omega)
$$

where

$$
\beta(\omega)=\omega \frac{\sqrt{\varepsilon_{e f f}}}{c_{\mathrm{o}}} .
$$


The loss component $\alpha(\omega)$, in decibels per metre, $\mathrm{dB} / \mathrm{m}$, are dependent on the conduction loss, $\alpha_{c}(\omega)$, given by

$$
\begin{gathered}
\alpha_{c}(\omega) \simeq 8.686 \frac{R_{S}(\omega)}{w Z_{\mathrm{o}}}, \\
R_{S}(\omega)=\frac{1}{\sigma_{c} \delta(\omega)}, \\
\delta(\omega)=\sqrt{\frac{2}{\omega \mu_{c} \sigma_{c}}},
\end{gathered}
$$

and on the dielectric loss, also in $\mathrm{dB} / \mathrm{m}$, is given by

$$
\begin{gathered}
\alpha_{d}(\omega) \simeq 27.3 \frac{\left(\varepsilon_{e f f}-1\right) \varepsilon_{r}}{\left(\varepsilon_{r}-1\right) \varepsilon_{e f f}} \frac{\omega \tan \theta}{2 \pi u}, \\
\tan \theta=\frac{\sigma}{\omega \varepsilon} .
\end{gathered}
$$

Equation (2.23.1), expresses the conductor loss, in terms of the frequency dependent skin resistance, $R_{S}(\omega)$, which is a function of the conductor's conductivity, $\sigma_{c}$, and skin depth, $\delta(\omega)$. The frequency dependent skin depth is also a function of the conductor conductivity and permeability, $\mu_{c}$. On the other hand, equation (2.24.1) expresses the dielectric loss as a function of frequency, relative permittivity, $\varepsilon_{r}$, effective relative permittivity, $\varepsilon_{\text {eff }}$, wave velocity, $u$, and loss tangent, $\tan \theta$.

Finally, writing $\alpha(\omega)$ in nepers per meter $\mathrm{Np} / \mathrm{m}$ requires, as described in Appendix A, the following conversion:

$$
\alpha(\omega)=\frac{\alpha_{c}(\omega)+\alpha_{d}(\omega)}{20 \log (e)}
$$




\subsection{Conclusion}

This chapter provides equations for determining the primary current distributions on the microstrip line that will be used for the determination of electric fields in the following chapter. In particular, with reference to Figure 2.4, expressions for the differential line currents $\hat{I}_{d}$ and polarisation currents $\hat{I}_{p o l}$ were presented for an arbitrarily loaded TL. Further the propagation constant, $\gamma(\omega)$, and characteristic impedance, $Z_{\mathrm{o}}$, required in the calculations of the currents are presented.

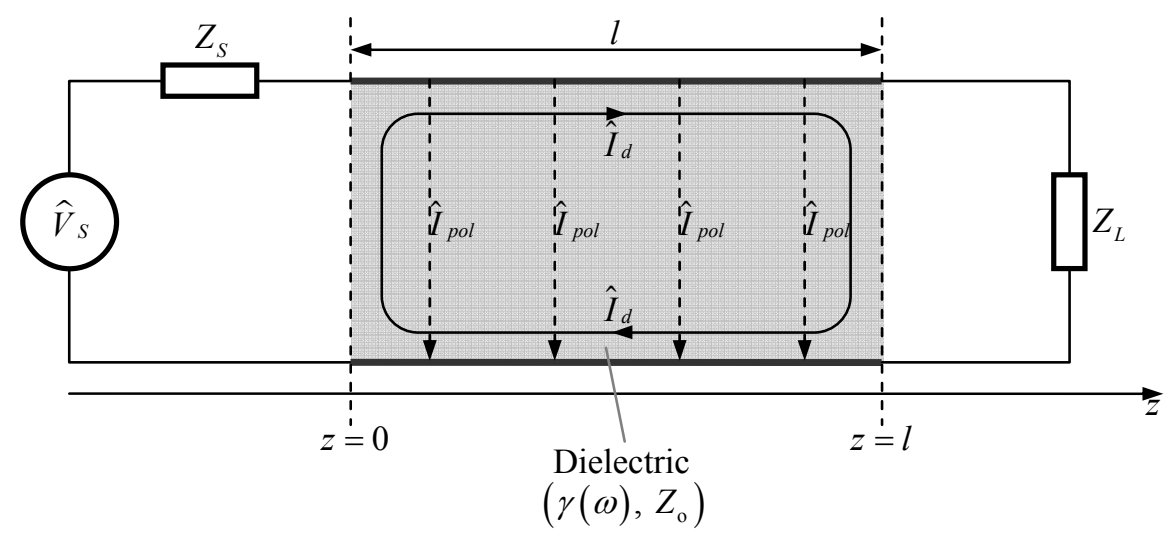

Figure 2.4 Microstrip currents. 


\section{Chapter 3. Simple Radiation Models}

\subsection{Introduction}

This chapter concerns the prediction of the electric field emissions for a microstrip line based on the current distribution obtained in Chapter 2. Two methods for computing the electric field, namely the Hertzian dipole and the Schelkunoff filament method are considered. Modelling with Hertzian dipoles, for linear structures, can be classified as a semi-numerical technique although the electric field equations for the dipole are analytic in nature. In contrast, Schelkunoff's method is purely analytical. When modelling a microstrip as defined in Chapter 1, the Hertzian dipole method is able to include the dielectric polarisation effects. A modelling technique utilising Schelkunoff's derivations requires the TL currents to have the free space wavelengths so that the radiation integral can simplify into a closed form analytic expression. Thus, while the Schelkunoff method is able to show directly influences of electrical parameters such as terminations on radiation characteristics, it cannot predict the effect of polarisation currents in the dielectric substrate. The Hertzian dipole method, being semi-numerical is able to capture the effects from polarisation currents. It is therefore possible, by utilising both methods, to gain useful insight into the radiation characteristics.

To gain confidence in the electric field modelling techniques, the radiation from two simple structures, namely a travelling wave current and a closed loop current, will be introduced and guidelines regarding segmentation for the Hertzian method will be given. Finally the Hertzian dipole method will be applied to the microstrip presented in Chapter 1.

The following key topics are covered:

1. The use of Hertzian dipole and Schelkunoff current filaments as antenna building blocks, and their limitations and benefits.

2. A comparison between the two modelling methods, in free space, for a simple travelling current and a closed loop. For the closed loop the effects from the 
image, end segments and dielectric on radiated emission are investigated by progressively building up the loop from a simple line model. Guidelines for segmentation when using the Hertzian method are given.

\subsection{Electric Field from Currents}

By definition, the electric field at a point is the force on a unit test charge at that point. It can be calculated by a combination of scalar potential, $\hat{V}$, and magnetic vector potential, $\widehat{\mathbf{A}}$ given by $[2,14,16,23]$

$$
\widehat{\mathbf{E}}=-\nabla \widehat{V}-j \omega \widehat{\mathbf{A}}
$$

If all sources $\hat{\rho}_{v}$ and $\hat{\mathbf{J}}$ are contained in a volume, $v$, the value of $\widehat{V}$ and $\widehat{\mathbf{A}}$, at a distance $R$ away from these source, are given by

$$
\begin{gathered}
\widehat{V}=\frac{1}{4 \pi \varepsilon} \int_{v} \frac{\hat{\rho}_{v} e^{-j \beta R}}{R} d v, \\
\widehat{\mathbf{A}}=\frac{\mu}{4 \pi} \int_{v} \frac{\hat{\mathbf{J}} e^{-j \beta R}}{R} d v,
\end{gathered}
$$

where

$$
R=\sqrt{\left(x-x^{\prime}\right)^{2}+\left(y-y^{\prime}\right)^{2}+\left(z-z^{\prime}\right)^{2}},
$$

in Cartesian coordinates, and $x^{\prime}, y^{\prime}$ and $z^{\prime}$ represent the source location.

The relationship between charge density, $\hat{\rho}_{v}$, and current is given by the continuity equation:

$$
\nabla \cdot \hat{\mathbf{J}}=-j \omega \hat{\rho}_{v}
$$

As can be seen from equations (3.2.1), (3.2.2) and (3.4), the scalar potential is a function of the charge distribution, which is a function of current density. Further the magnetic potential is also a function of the current density. Therefore from these 
equations, the electric field in equation (3.1) can be expressed in terms of current sources only. Thus the current distributions derived in Chapter 2 are sufficient, for a current carrying conductor for any structure, for the purpose of determining radiated emissions. Both the Hertzian's dipole and the Schelkunoff's filament method provide the link between a current distribution and the radiated field.

\subsection{Hertzian Dipole Model}

A Hertzian dipole is an infinitesimally small current carrying element as shown in Figure 3.1. It is shown as having length $d z$. The current, $\hat{I}$, is assumed to have a uniform magnitude and phase over this infinitesimal length.

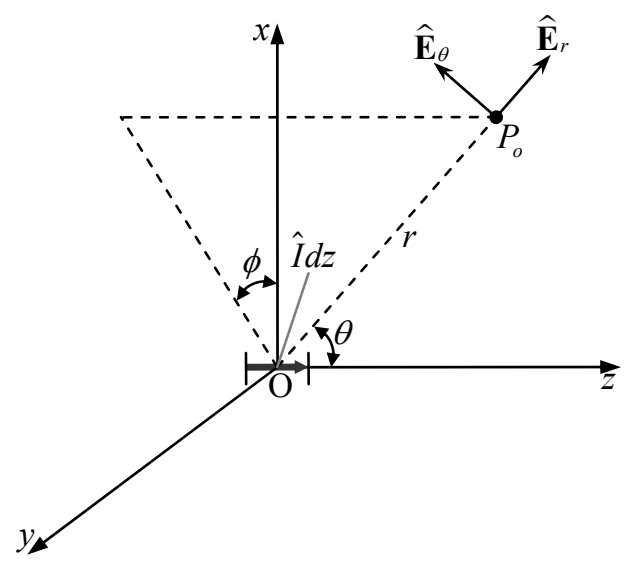

Figure 3.1 An oscillating current carrying Hertzian dipole.

From [15] with reference to Figure 3.1 the electric field, at observation point, $P_{o}$, in spherical coordinates is given by:

$$
\begin{gathered}
\widehat{E}_{r}=\frac{\eta \hat{I} d z}{2 \pi}\left(\frac{1}{r^{2}}-\frac{j}{\beta r^{3}}\right) e^{-j \beta r} \cos \theta, \\
\widehat{E}_{\theta}=\frac{\eta \hat{I} d z}{4 \pi}\left(\frac{j \beta}{r}+\frac{1}{r^{2}}-\frac{j}{\beta r^{3}}\right) e^{-j \beta r} \sin \theta, \\
\widehat{E}_{\phi}=0 .
\end{gathered}
$$


The intrinsic impedance, $\eta$, is defined in terms of the permeability, $\mu$, and the permittivity $\varepsilon$, as

$$
\eta=\sqrt{\frac{\mu}{\varepsilon}} .
$$

In free space the following substitutions are used

$$
\begin{gathered}
\eta=\eta_{\mathrm{O}}=\sqrt{\frac{\mu_{\mathrm{O}}}{\varepsilon_{\mathrm{O}}}}, \\
\beta=\beta_{\mathrm{O}}=\frac{2 \pi}{\lambda_{\mathrm{O}}},
\end{gathered}
$$

with the phase constant having units of radians per metre.

The transition between the near and far fields defined by distance $r_{\mathrm{O}}$, as given in Appendix B is

$$
r_{\mathrm{O}}=\frac{\lambda}{2 \pi}
$$

For $r=r_{\mathrm{O}}$ the magnitude contributions from the $r^{-1}$ and $r^{-2}$ terms are the same.

It is possible to use the analytic result from equations (3.5.1) and (3.5.2) together with segmentation and superposition to determine the fields created by complex current distributions. For example, a current on a TL structure can be segmented into many small dipoles and the entire radiation determined by summing up the contributions of the individual dipoles according to these equations at a particular observation point. Note that care must be taken in translating the many dipole equations, all referenced to their own local coordinate systems, into a global coordinate system so that the components of the electric field can be added up. This method is referred to the "Hertzian dipole method" in this thesis. 
Hertzian dipoles are idealised structures, which are often used as building blocks for antenna structures. To achieve this they are assumed to have a small but finite length, $d z$. Their most useful feature is the fact that their electric field at any point in space can still be calculated using the analytic equations for the infinitesimal dipole to a good approximation.

Two major criteria must be met when constructing an antenna comprising of Hertzian dipoles. The first criterion requires that the length of each dipole segment is much shorter than the wavelength at the maximum frequency of interest. This is to satisfy the assumption of a uniform current carrying element. A general rule of thumb that is most commonly used incorporates a factor of ten into the calculations:

$$
d z \leq \frac{\lambda}{10}
$$

The second criterion requires that the distance of the observation point from each dipole, $r_{o}$, be large compared to the length of the dipoles, such that effects from current discontinuities (i.e. charge singularities) at the dipole joints are minimised. A useful approximation that will be demonstrated in Section 3.5 is that the observation point be further away from the structure than the length of the dipole used, i.e.:

$$
r_{o}>d z
$$

\subsection{Schelkunoff Model}

In this section, a summary of Schelkunoff's analytical derivations are presented. For a given geometry as illustrated in Figure 3.2 it can be shown that the electric field, from a filament of sinusoidal current can be expressed in terms of the current and its derivatives at the ends of the filament. There is no need to segment the wire as in the Hertzian dipole method. Hence, obtaining radiation field results is not only computationally quicker, but also more insightful as the effect of line length and frequency are seen in the equations. 


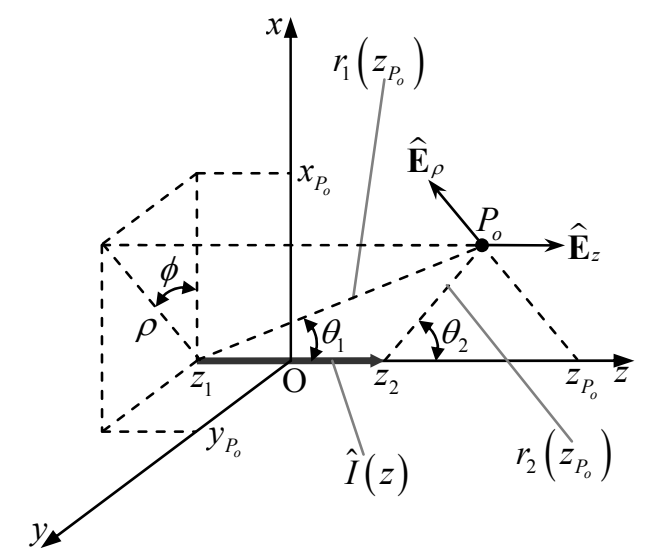

Figure 3.2 Current carrying wire in Cartesian and cylindrical coordinates.

Schelkunoff's equations in [13] have recently been applied in the area of EMC, [26]. In relation to Figure 3.2 a compact matrix form for the electric field in cylindrical coordinates, with derivations given in Appendix B, may be written as [8]

$$
\begin{aligned}
& \widehat{\mathbf{E}}=\left.K\left[\mathbf{G} \hat{\mathbf{I}}_{D}\right]\right|_{z_{e}=z_{2}} ^{z_{e}=z_{1}}, \\
& \widehat{\mathbf{E}}=\left[\begin{array}{c}
\widehat{E}_{z} \\
\widehat{E}_{\rho} \\
\widehat{E}_{\phi}
\end{array}\right], \\
& K=\frac{j}{4 \pi \omega \varepsilon_{\mathrm{o}}}, \\
& \mathbf{G}=e^{-j \beta r_{z_{e}}\left(z_{\mathrm{p}_{o}}\right)}\left[\begin{array}{cc}
\frac{\left(1+j \beta r_{z_{e}}\left(z_{P_{o}}\right)\right) \cos \theta_{z_{e}}}{r_{z_{e}}^{2}\left(z_{P_{o}}\right)} & -\frac{1}{r_{z_{e}}\left(z_{P_{o}}\right)} \\
\frac{\sin ^{2} \theta_{z_{e}}-j \beta r_{z_{e}}\left(z_{P_{o}}\right) \cos ^{2} \theta_{z_{e}}}{\rho r_{z_{e}}\left(z_{P_{o}}\right)} & \frac{\cos \theta_{z_{e}}}{\rho} \\
0 & 0
\end{array}\right], \\
& \hat{\mathbf{I}}_{D}=\left[\begin{array}{l}
\hat{I}\left(z_{e}\right) \\
\hat{I}^{\prime}\left(z_{e}\right)
\end{array}\right] .
\end{aligned}
$$


Equation (3.11.1) gives the electric field as a function of the current distribution, $\hat{\mathbf{I}}_{D}$ evaluated at the ends of the current carrying wire, and the geometry, $\mathbf{G}$, and a scaling term, $K$. The current distribution can be obtained from TL model if the wire is treated as one conductor of a TL. From equation (3.11.4) and with reference to Figure 3.2 the radial observation distance functions in the geometry matrix are defined as:

$$
r_{z_{e}}\left(z_{P_{o}}\right)=\sqrt{\rho^{2}+\left(z_{P_{o}}-z_{e}\right)^{2}}
$$

In equation (3.12) the variable $z_{P_{o}}$ defines the $z$-component variation of the observation point and $z_{e}$ for the ends. At the ends equation (3.12) then becomes

$$
\begin{aligned}
& r_{1}\left(z_{P_{o}}\right)=\sqrt{\rho^{2}+\left(z_{P_{o}}-z_{1}\right)^{2}}, \\
& r_{2}\left(z_{P_{o}}\right)=\sqrt{\rho^{2}+\left(z_{P_{o}}-z_{2}\right)^{2}} .
\end{aligned}
$$

In a similar way complex current distributions are analysed in the Hertzian method by adding up dipoles, it is possible to add up the effects of sinusoidal filamentary currents using Schelkunoff equations. Since the current distributions on thin wire antennas are naturally sinusoidal [13], this method, at a first glance appears to be very attractive. In this thesis the suitability of using Schelkunoff's equations for TL current structures is examined.

\subsection{Travelling Line Current}

The analysis of the entire microstrip is complicated by the presence of a dielectric medium. As a first step, the relationship between the currents and emissions from a straight thin wire in free space is considered. The first approach is to use the Hertzian method, whereby the wire structure is segmented into dipoles. In the second approach the integral method developed by Schelkunoff is used. Comparisons between both models are made and later extended to model emissions from a return path and then from TL loops. Literature studies $[10,12,18]$ show that there are 
models for computing the electric field of a square loop in free space but can be rather involved with no additional improvements in computational time.

In order to show the effect of charges and segmentation, consider the case of a travelling or phased current directed along the $z$-axis, with the given distribution:

$$
\hat{I}(z)=e^{-j \beta_{0} z}
$$

for $-l / 2 \leq z \leq l / 2$. The geometry is illustrated in Figure 3.3.

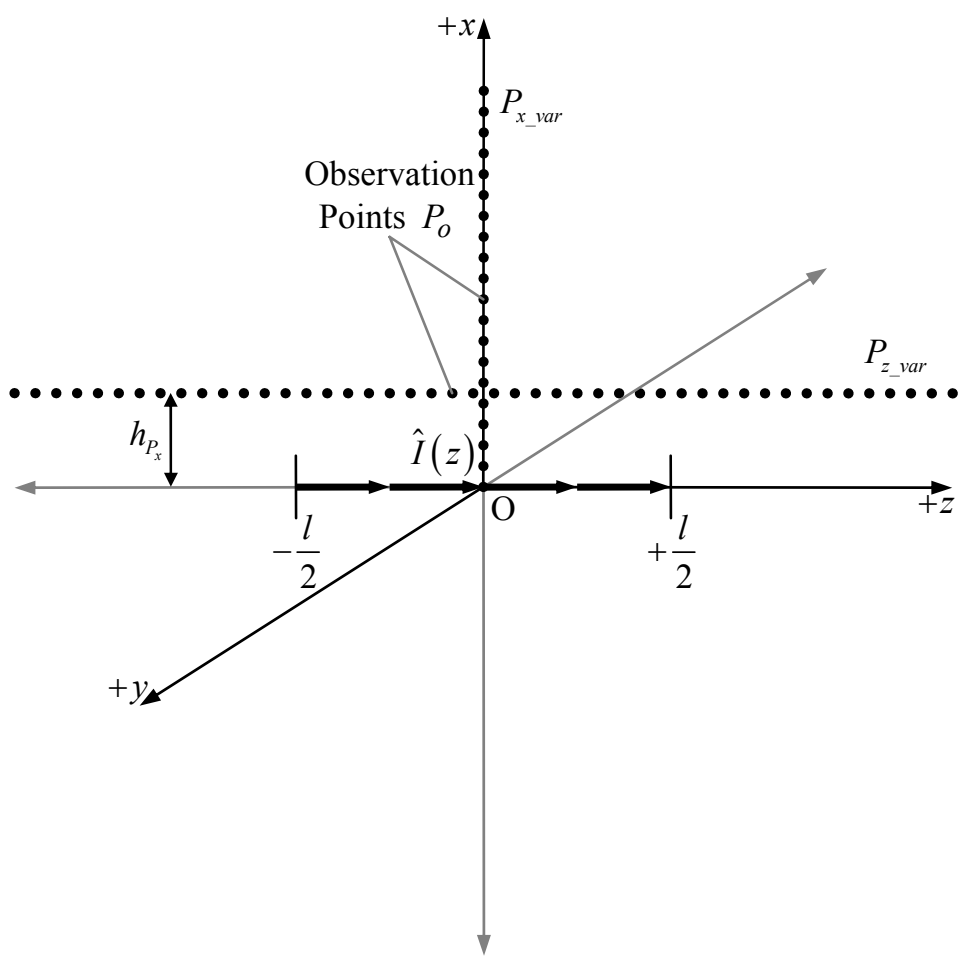

Figure 3.3 Current distribution and observation points for a line.

The first case would be to do an observation sweep, $P_{z_{-} v a r}$, on the $x z$-plane, comparing both the Hertzian dipole method and Schelkunoff method. This is done at a frequency of $500 \mathrm{MHz}$ and at a distance of $h_{P_{x}}=1 \mathrm{~mm}$ from the current filament. The magnitude of the electric field, defined as

$$
E_{m a g} \triangleq \sqrt{\left|E_{x}\right|^{2}+\left|E_{y}\right|^{2}+\left|E_{z}\right|^{2}},
$$

is plotted in all the following figures. 
Figure 3.4 shows that the Hertzian model gives the same solution as Schelkunoff in the limit as the number of segments are increased to infinity. Furthermore, the plot shows that current discontinuities at the dipole joins cause high field strengths in their vicinity. Since the current flowing along a Hertzian dipole is assumed uniform, electric point charges relating to the change in current by going from one dipole to its neighbour give rise to these fictitious fields. It is demonstrated in Figure 3.4 that for 300 segments per $20 \mathrm{~cm}$ (i.e. for segment length of $0.7 \mathrm{~mm}$ ) this effect is very small for an observation distance of $1 \mathrm{~mm}$. Thus equation (3.10) is demonstrated to give a suitable guideline for segmentation.

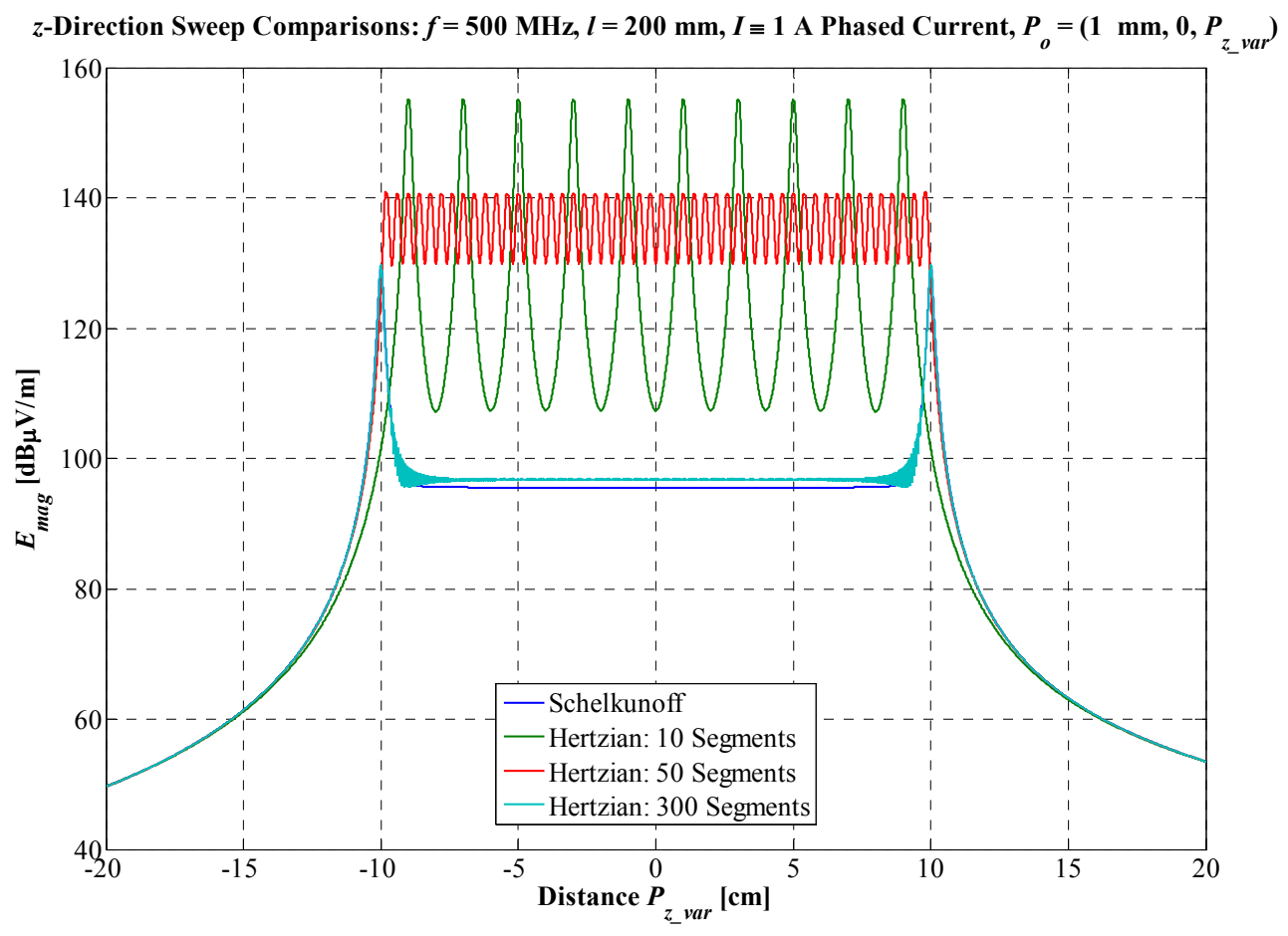

Figure $3.4 z$-direction sweep comparisons.

Note that both techniques give peaks in the electric field at the ends of the current filament. These peaks are caused by the discontinuity in the current at these points. These peaks are artificial, as currents in a physical system go to zero at the ends of unconnected wires. They illustrate the importance of "closing the current loop" when modelling TL. It will be shown in the following section that the current on the vertical risers at the ends of a TL is very important to include for this reason, although its physical size may seem to be insignificant compared to the length of the line. 
The second case would be to do an observation sweep, $P_{x_{-} v a r}$, along the $x$-axis.

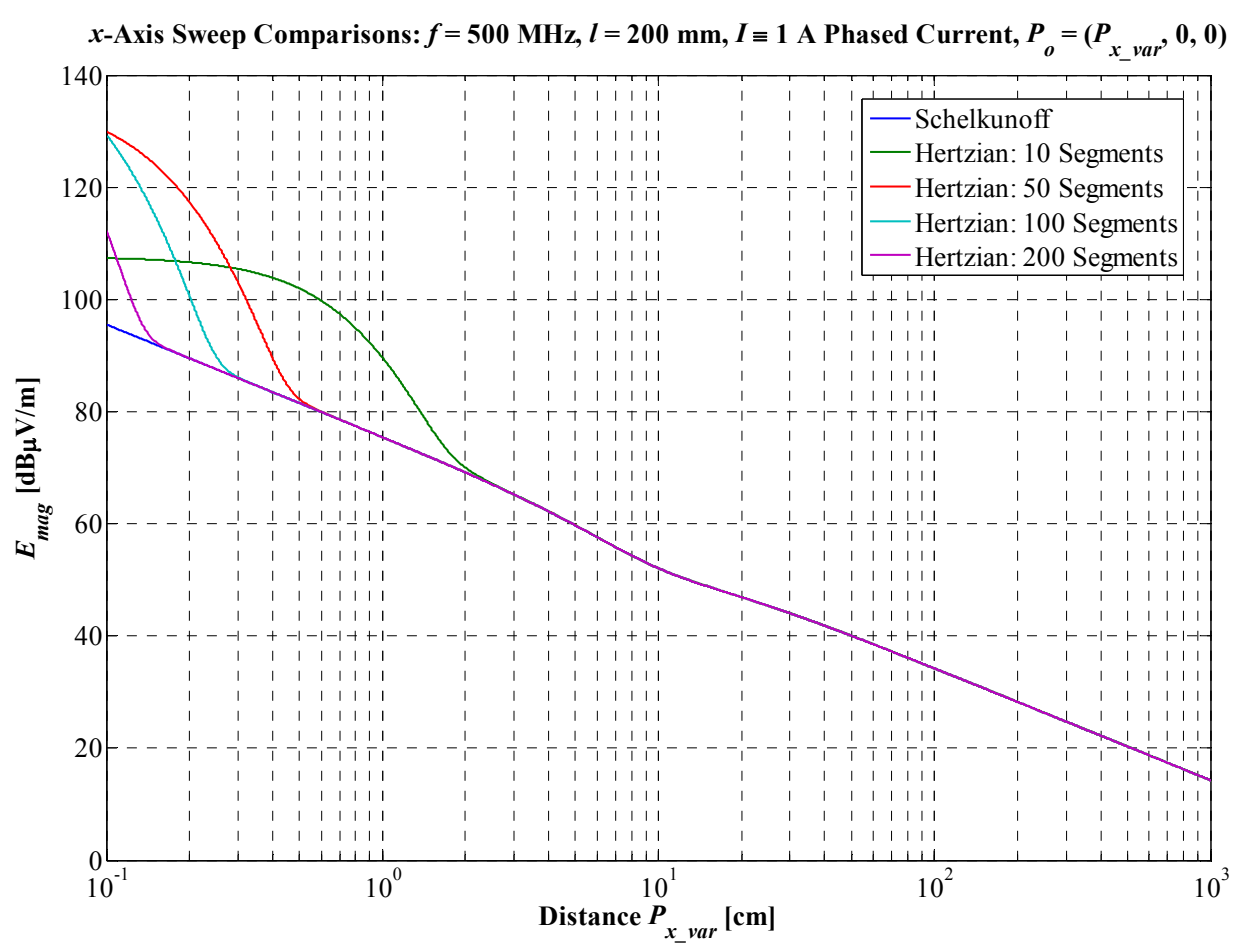

Figure $3.5 x$-direction sweep comparisons.

From Figure 3.5, at distances close to the line source, the calculated field increases. As the number of Hertzian dipole segments increase, the Hertzian converges to the Schelkunoff result. Another good result is the frequency sweep shown in Figure 3.6. Again it is seen that the dipole results converge to the Schelkunoff results, if enough segments are used. It can be seen that using only 5 segments gives good agreement up to around $2 \mathrm{GHz}$. Above this frequency discrepancies begin to appear. From the plots given in Figure 3.4, Figure 3.5 and Figure 3.6, it can be seen that a $20 \mathrm{~cm}$ line will require roughly 200 segments for good electric field calculations for observation points as close to $1 \mathrm{~mm}$ to the line. As shown this segmentation is also easily adequate up to $1 \mathrm{GHz}$, which is the highest frequency considered in this study. For this segmentation both criterions given by equations (3.9) and (3.10) are satisfied. 


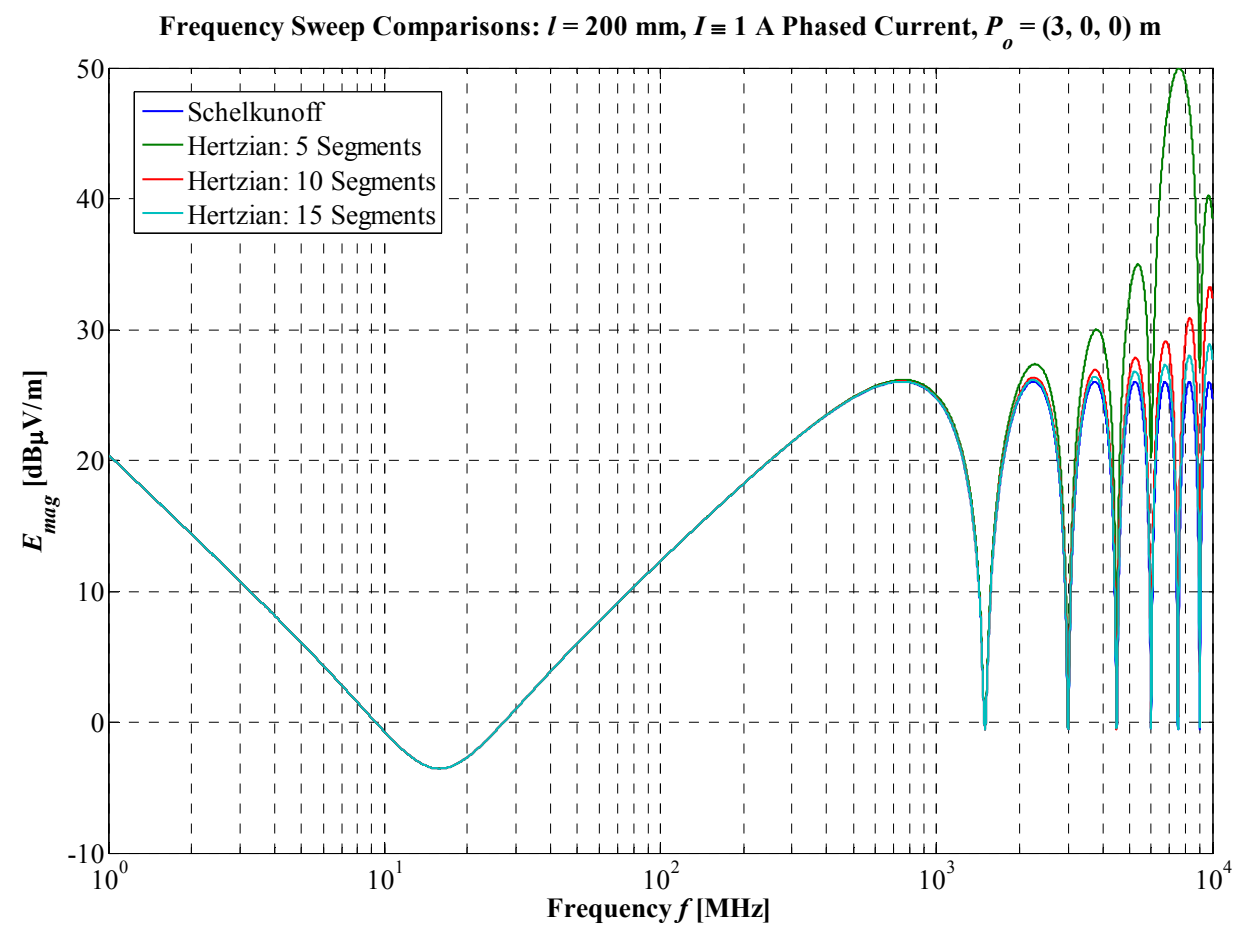

Figure 3.6 Frequency sweep comparisons.

\subsection{Closed Loop Transmission Line Radiation}

In [13] Schelkunoff presented closed form expressions for the electric field radiation for a line carrying sinusoidal currents. It is known that TL current distributions are sinusoidal, being composed of a sum of forward and backward travelling waves. Therefore, it is possible to extend Schelkunoff's results to predict the radiation field of free space TL structures. However, a closed TL loop requires modelling of the return path and end segments. The radiation from these end segments is not present in Schelkunoff's model and as yet there is no convenient way of including these effects in his equations. This is something to be investigated for future work, and radiation analysis from a symmetrical microstrip can then be easily obtained utilising the method of images.

Unlike the Hertzian dipole, Schelkunoff's equations allow currents to be made zero at the ends of the line, as would naturally exist for antennas. However, it also caters for non-zero end currents, but in this case care must be taken to complete the current loop, or charge build-up must be addressed. This can be dealt with by the inclusion of end segments. 
Similarly to Figure 3.3, consider the case for a loop as shown in Figure 3.7. For this example, it is assumed that the current on the TL, given by $\hat{I}(x, z)$ in the $y=0$ plane is as follows:

The line currents, in the $z$-direction, are:

$$
\begin{array}{ll}
\text { Trace: } & \hat{I}(+h, z)=e^{-j \beta_{\mathrm{0}} z},[\mathrm{~A}], \\
\text { Image: } & \hat{I}(-h, z)=-e^{-j \beta_{\mathrm{0}} z},[\mathrm{~A}] .
\end{array}
$$

The end segment currents, in the $x$-direction, are:

Left End: $\quad \hat{I}\left(0,-\frac{l}{2}\right)=e^{+j \beta_{0} \frac{l}{2}},[\mathrm{~A}]$

$$
\text { Right End: } \quad \hat{I}\left(0,+\frac{l}{2}\right)=-e^{-j \beta_{0} \frac{l}{2}},[\mathrm{~A}]
$$

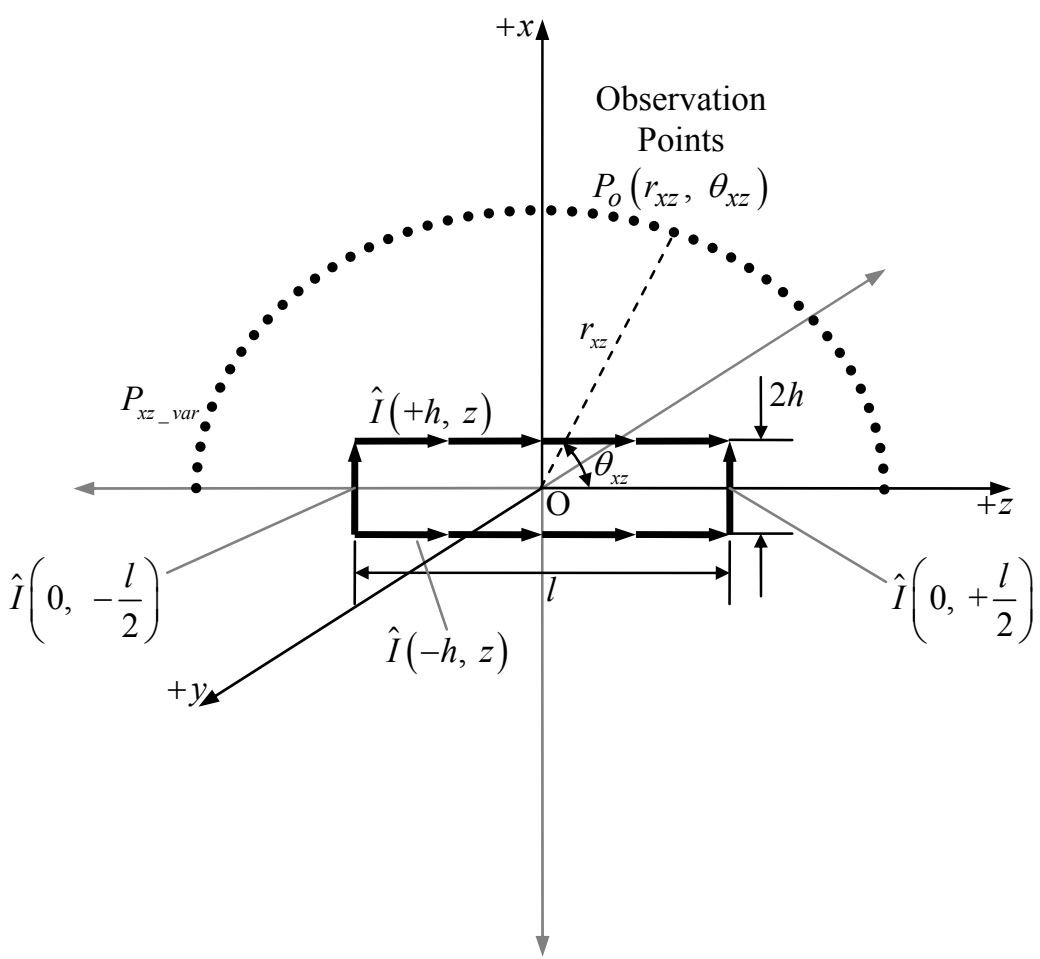

Figure 3.7 Current distributions and observation points for loop. 
From Figure 3.7 the line carrying $\hat{I}(-h, z)$ is the return, or called the image, and the vertical risers at $z=-l / 2$ and $z=+l / 2$ are called end segments. Note that the use of image currents to complete the loop assumes an infinite perfect ground plane (AS5). For validation by measurement in Chapter 4 considerable care is taken to create a very large ground plane by using copper shim and copper tape to electrically connect the finite ground of the TL structure to the semi-anechoic chamber's ground. Some preliminary measurement results have shown that if the ground plane is truncated, it will cause an increase in radiated emissions at low frequencies $(<400 \mathrm{MHz})$.

For the loop structure with currents as indicated, comparisons are made for the Hertzian dipole and the Schelkunoff method. These were made by progressively building the loop to show the effects of (i) adding an image line [20], and (ii) adding the end segments [3]. This is done for two arbitrarily chosen frequencies, one in the low range $(f=30 \mathrm{MHz})$ and the other in the high range $(f=1 \mathrm{GHz})$ and with $h=1.5 \mathrm{~mm}$. A distance close to the structure $\left(r_{x z}=12 \mathrm{~cm}\right)$ is chosen so that all the charge related effects can be observed in the electric field. A polar sweep in the angular range $0^{\circ}<\theta_{x z}<180^{\circ}$ is carried out to capture the fields at the ends and about the TL.

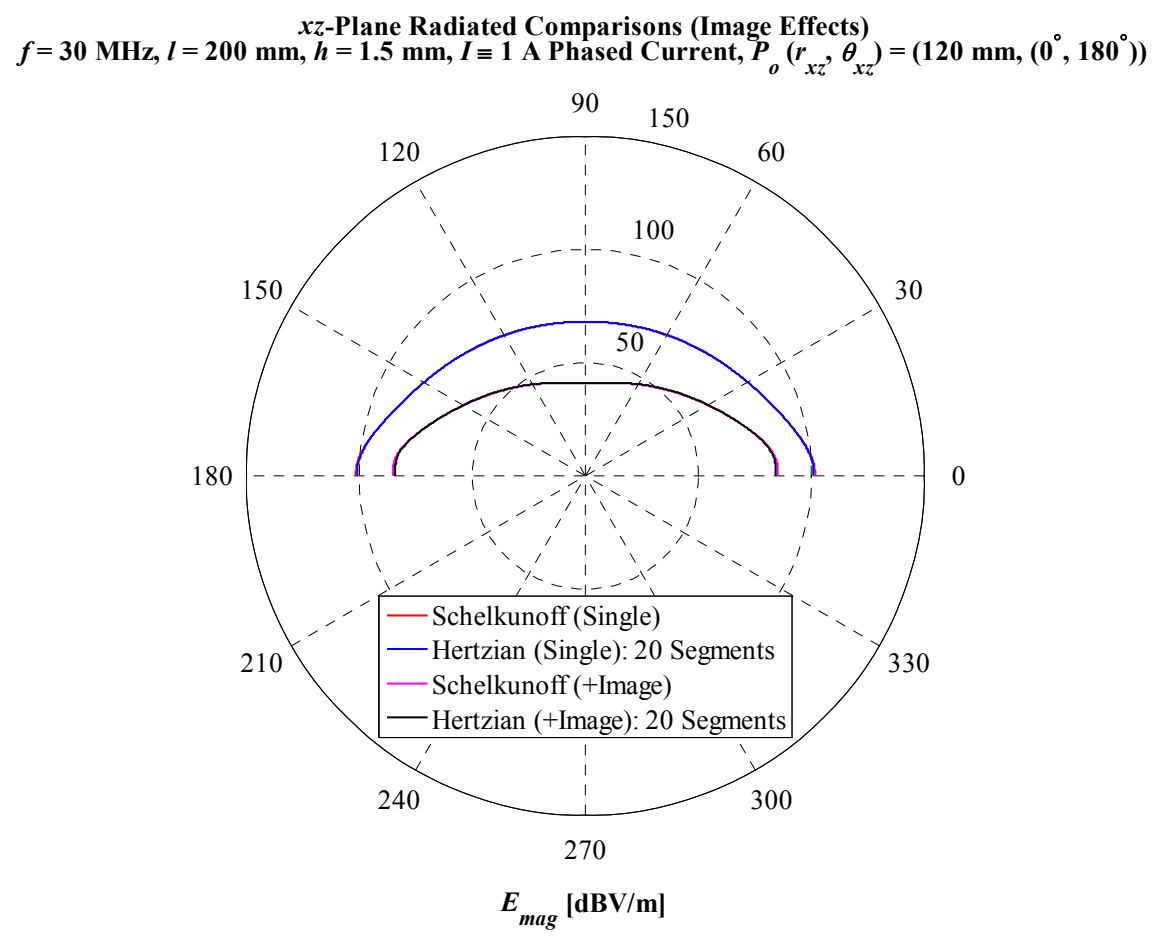

Figure 3.8 $x z$-plane observation image effects comparisons at $30 \mathrm{MHz}$. 
Figure 3.8 shows that at $30 \mathrm{MHz}$ the Schelkunoff method agrees with the Hertzian method with 20 segments, at a radial observation sweep from the origin, $\mathrm{O}$, of $r_{x z}=12 \mathrm{~cm}$. The emphasis of this plot is to show the considerable difference of adding an image. In this example the magnitude of the field of the single line is reduced by about $40 \mathrm{~dB}$ around $\theta_{x z}=90^{\circ}$. It is also observed that there is a higher field strength in the $z$-direction (end on to TL) compared to the $x$-direction (above line) because the ends of the lines are not "closed", allowing an accumulation of charge to occur.

The next Figure 3.9 again shows good agreement between the Schelkunoff and Hertzian method at $1 \mathrm{GHz}$. It is seen that there is still a considerable difference between the line with and without its image, however the image is no longer quite as effective in reducing radiation, especially at around $\theta_{x z}=90^{\circ}$.

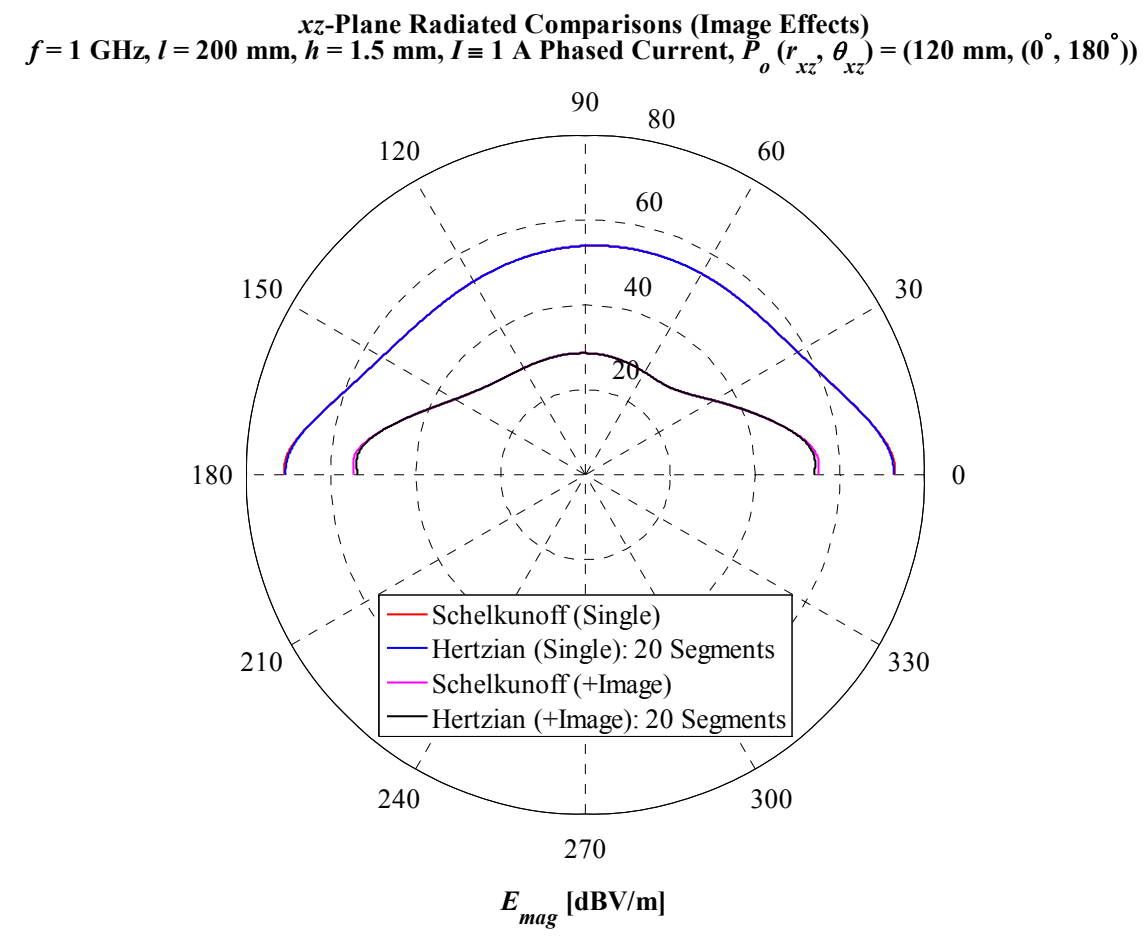

Figure 3.9 $x z$-plane observation image effects comparisons at $1 \mathrm{GHz}$. 
Consider the case now when the end segments are added. Here the Hertzian dipole method is used to show the effects due to them for a frequency of $30 \mathrm{MHz}$ and 1 GHz. In Figure 3.10 addition of the end segments further reduces the magnitude of the electric field as these local charges at the ends are "capped off". At $30 \mathrm{MHz}$ the radiated emissions have symmetry. At $1 \mathrm{GHz}$, Figure 3.11 shows that the end segments cause an unsymmetrical radiation pattern at one end of the line compared to the other. Such an effect is to be expected from a travelling wave $[4,11]$ at high frequencies. This effect, however only becomes visible when the high fields due to the charges at the end of the lines are removed by closing the current loop.



Figure 3.10 $x z$-plane observation end effects comparison at $30 \mathrm{MHz}$. 


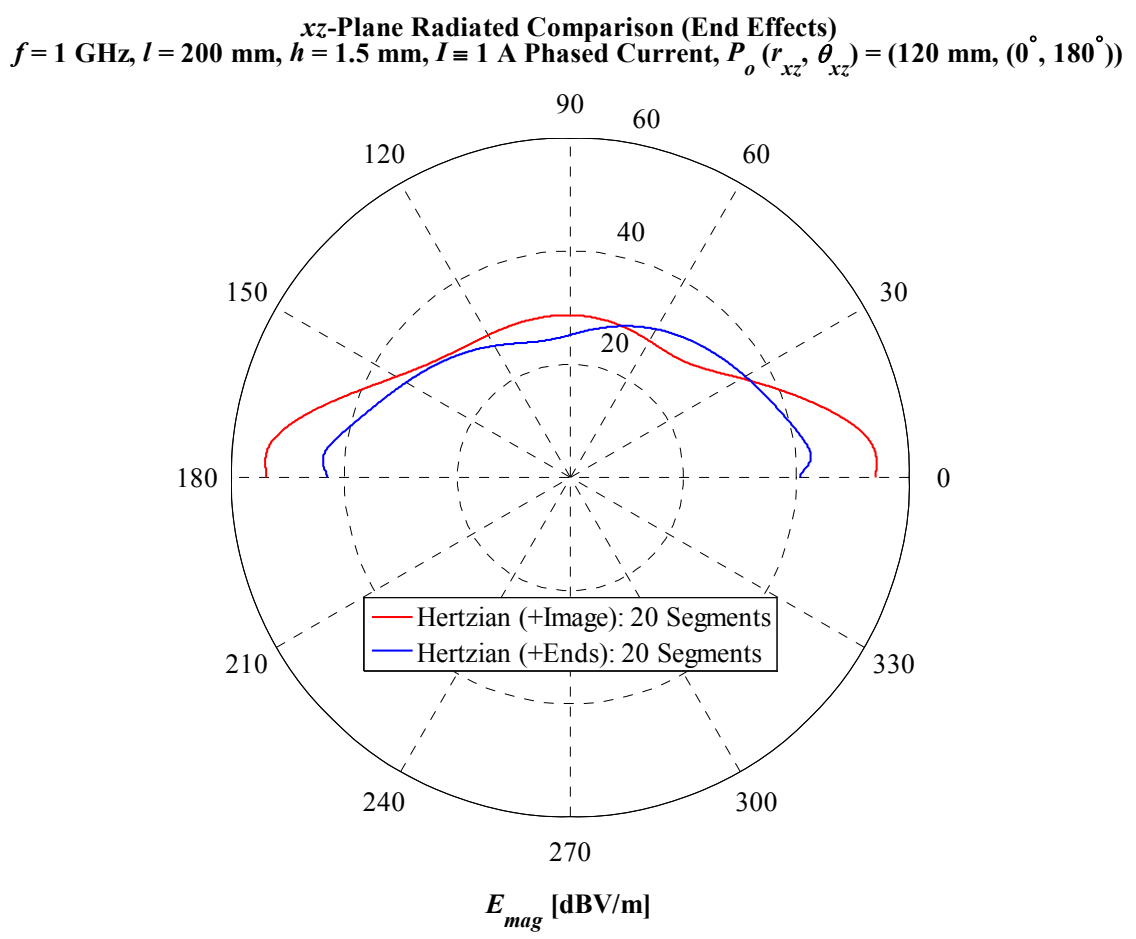

Figure 3.11 $x z$-plane observation end effects comparison at $1 \mathrm{GHz}$.

\subsection{Current Loop with Dielectric}

In this section the current loop of the previous section is extended to include a dielectric between the TL conductors. The influence of the dielectric on the radiation characteristic is determined. It is assumed that the dielectric has an effective permittivity, $\varepsilon_{\text {eff }}$ introduced in the previous chapter, such that the TL wave number is

$$
\beta_{l}=\sqrt{\varepsilon_{\text {eff }}} \beta_{\mathrm{o}} .
$$

The first thing to note is that Schelkunoff's analytical expressions assuming that

$$
\beta_{l}=\beta_{\mathrm{o}}
$$

are therefore no longer applicable, as it was this key assumption that enabled Schelkunoff to derive the closed form equations. Furthermore, the dielectric causes polarisation currents to flow between the trace and the ground. The radiation from these currents cannot be predicted by Schelkunoff as they are parallel dipoles and not co-linear, and are not phased with $\beta_{0}$. 


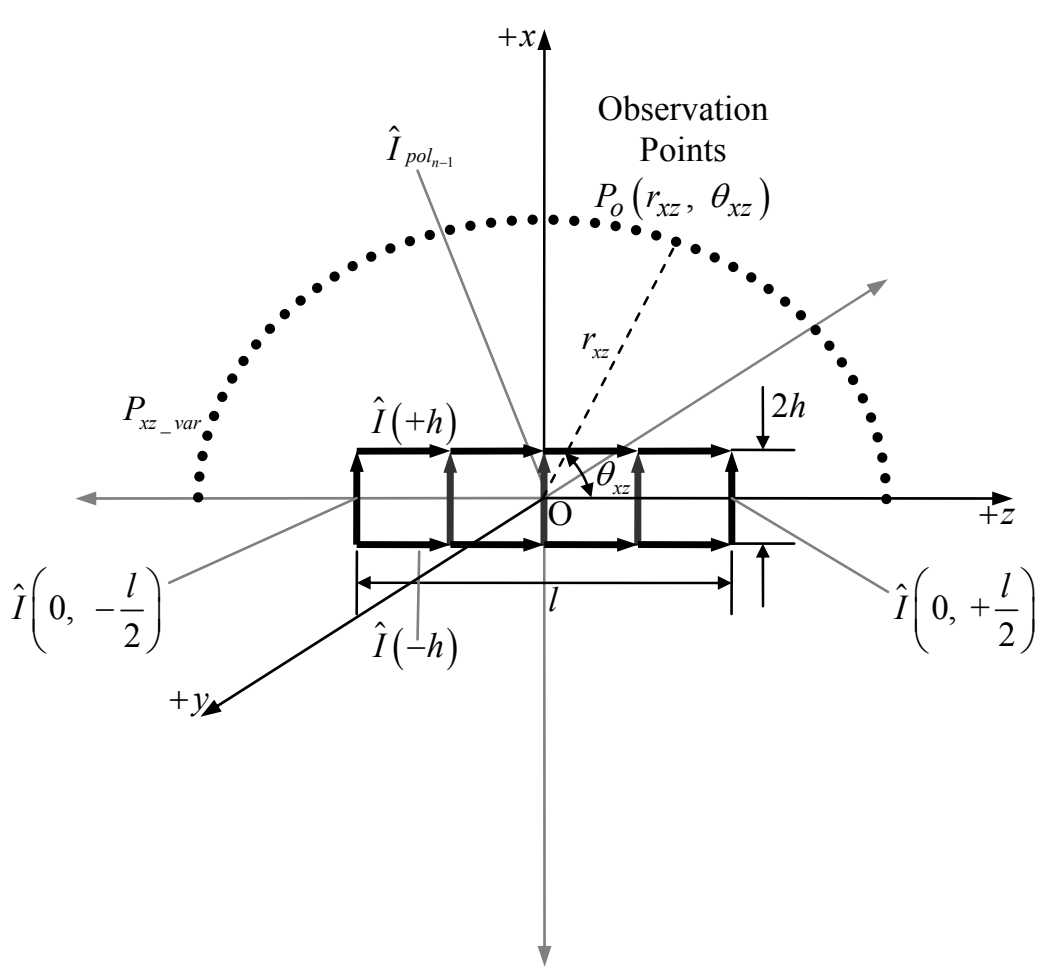

Figure 3.12 Current distributions and observation points for a loop.

Consider this loop shown in Figure 3.12. Assume the line currents are given as follows:

The line currents, in the $z$-direction, are:

$$
\begin{array}{ll}
\text { Trace: } & \hat{I}_{n}(+h)=e^{-j \beta_{l} z_{n}},[\mathrm{~A}], \\
\text { Image: } & \hat{I}_{n}(-h)=-e^{-j \beta_{l} z_{n}},[\mathrm{~A}],
\end{array}
$$

where $z_{n}$ is the centre location of dipole $n$ and is defined as:

$$
\begin{gathered}
z_{n}=\left\{-\frac{l}{2}+\frac{\Delta_{z}}{2},-\frac{l}{2}+\frac{3 \Delta_{z}}{2}, \cdots,-\frac{l}{2}+\frac{(2 N+1) \Delta_{z}}{2}\right\}, \\
\Delta_{z}=\frac{l}{N},
\end{gathered}
$$

where $\Delta_{z}$ is the segment length and $N$ is the total number of segments. 
The end segment currents, in the $x$-direction, are:

Left End:

$$
\hat{I}\left(0,-\frac{l}{2}\right)=e^{+j \beta_{l} \frac{l}{2}},[\mathrm{~A}],
$$

$$
\text { Right End: } \quad \hat{I}\left(0,+\frac{l}{2}\right)=-e^{-j \beta_{l} \frac{l}{2}},[\mathrm{~A}] \text {. }
$$

The polarisation currents, in the $x$-direction, are:

$$
\hat{I}_{p o l_{n}}=\left(\hat{I}_{n+1}-\hat{I}_{n}\right)\left(1-\frac{1}{\varepsilon_{r}}\right),[\mathrm{A}]
$$

where $n \in[1, N-1]$ and $N$ is the total number of segments. The polarisation currents are modelled by $(N-1)$ vertical dipoles, each having a length of $2 h$.

Figure 3.13 shows the radiation characteristics of the microstrip structure with $\varepsilon_{\text {eff }}=3.8$ at a frequency of $30 \mathrm{MHz}$. Two characteristics are shown. Both have the same line currents (derived using $\beta_{l}$ ); however only one includes the effects of the polarisation currents.

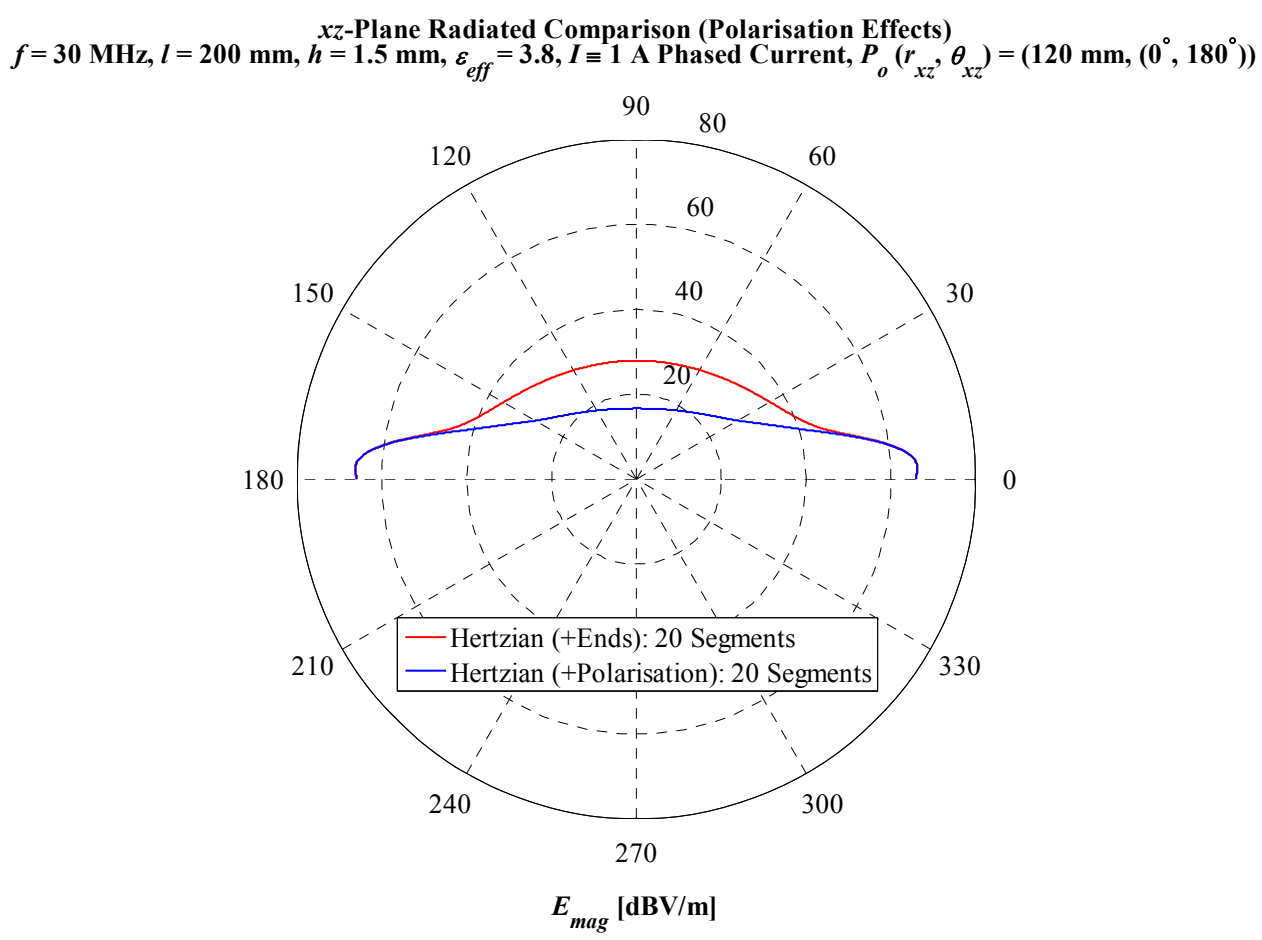

Figure 3.13 $x z$-plane observation polarisation effects comparison at $30 \mathrm{MHz}$. 
The radiation characteristics at $1 \mathrm{GHz}$ are shown in Figure 3.14.

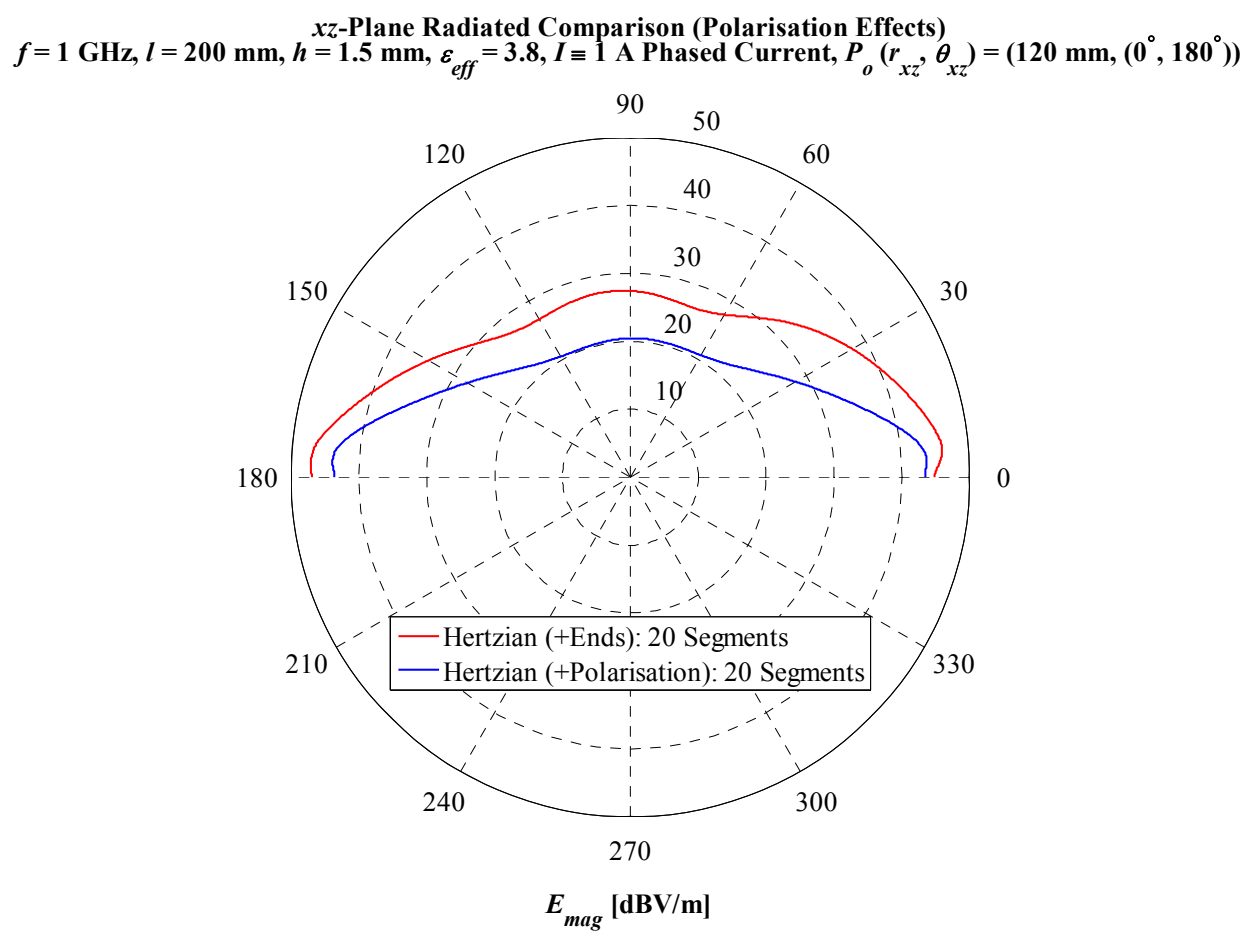

Figure 3.14 $x z$-plane observation polarisation effects comparison at $1 \mathrm{GHz}$.

At a higher frequency $(1 \mathrm{GHz})$ it is observed from Figure 3.14 that polarisation current reduce the fields for all observation angles. The difference is shown to be as much as $15 \mathrm{~dB}$. This means that dielectric polarisation currents are important as they may significantly change the radiation pattern.

\subsection{Microstrip with Arbitrary Termination}

In this section the model of the closed current loop with dielectric is applied to a real microstrip TL by adding a voltage source $\hat{V}_{S}$ to one end of the line $(z=-l / 2)$ and a load $Z_{L}$ to the other end $(z=+l / 2)$.

Trace currents in reality flow as a sheet on the underside of the trace due to the skin effect. In this model it is assumed, that for the purposes of radiation, this distribution in cross-section is sufficiently symmetrical so that the current can be modelled as a string of end-to-end dipoles centred at the middle of each segment (AP1). 


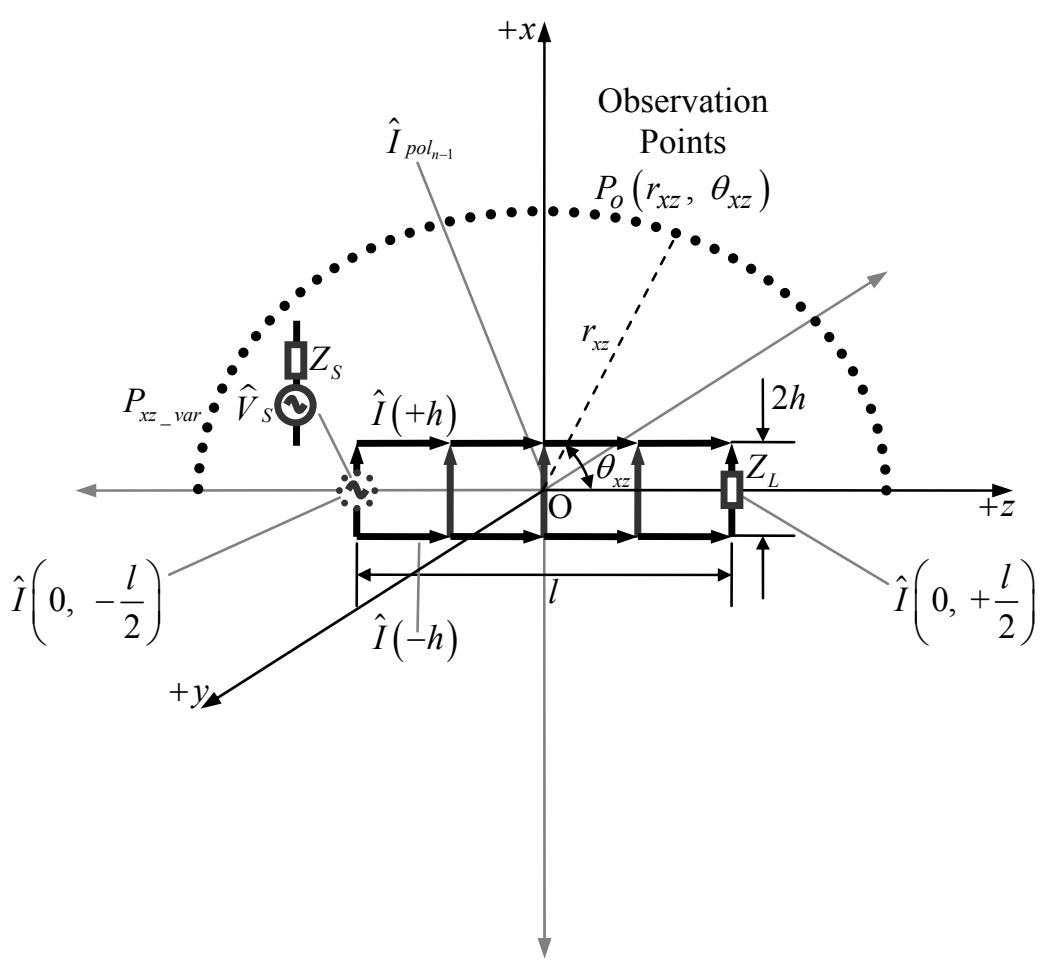

Figure 3.15 Current distributions and observation points for microstrip.

For $N$ dipoles the currents, in the $z$-direction, are:

Trace: $\quad \hat{I}_{n}(+h)=\frac{\hat{V}_{S}}{D}\left(Z_{\mathrm{o}} \cosh \gamma\left(z_{n}\right)+Z_{L} \sinh \gamma\left(z_{n}\right)\right), \quad[\mathrm{A}]$

Image: $\quad \hat{I}_{n}(-h)=-\frac{\hat{V}_{S}}{D}\left(Z_{\mathrm{o}} \cosh \gamma\left(z_{n}\right)+Z_{L} \sinh \gamma\left(z_{n}\right)\right), \quad[\mathrm{A}]$.

The end segment currents, in the $x$-direction, are:

$$
\begin{aligned}
& \text { Left End: } \quad \hat{I}\left(0,-\frac{l}{2}\right)=\frac{\hat{V}_{S}}{D}\left(Z_{\mathrm{o}} \cosh \gamma l+Z_{L} \sinh \gamma l\right),[\mathrm{A}], \\
& \text { Right End: } \\
& \qquad \hat{I}\left(0,+\frac{l}{2}\right)=\frac{\hat{V}_{S} Z_{\mathrm{o}}}{D},[\mathrm{~A}],
\end{aligned}
$$

where

$$
D=Z_{\mathrm{o}}\left(Z_{S}+Z_{L}\right) \cosh \gamma l+\left(Z_{\mathrm{o}}^{2}+Z_{S} Z_{L}\right) \sinh \gamma l .
$$

The polarisation currents, in the $x$-direction, are calculated using equation (3.23). 
Figure 3.16 shows simulation results for line with $\hat{V}_{S}=1, \varepsilon_{\text {eff }}=3.8$ and $Z_{S}=0$. The TL was loaded with a $22 \mathrm{pF}$ capacitor to simulate the input capacitance of the gate. Note that the termination has a significant effect on the radiation pattern.

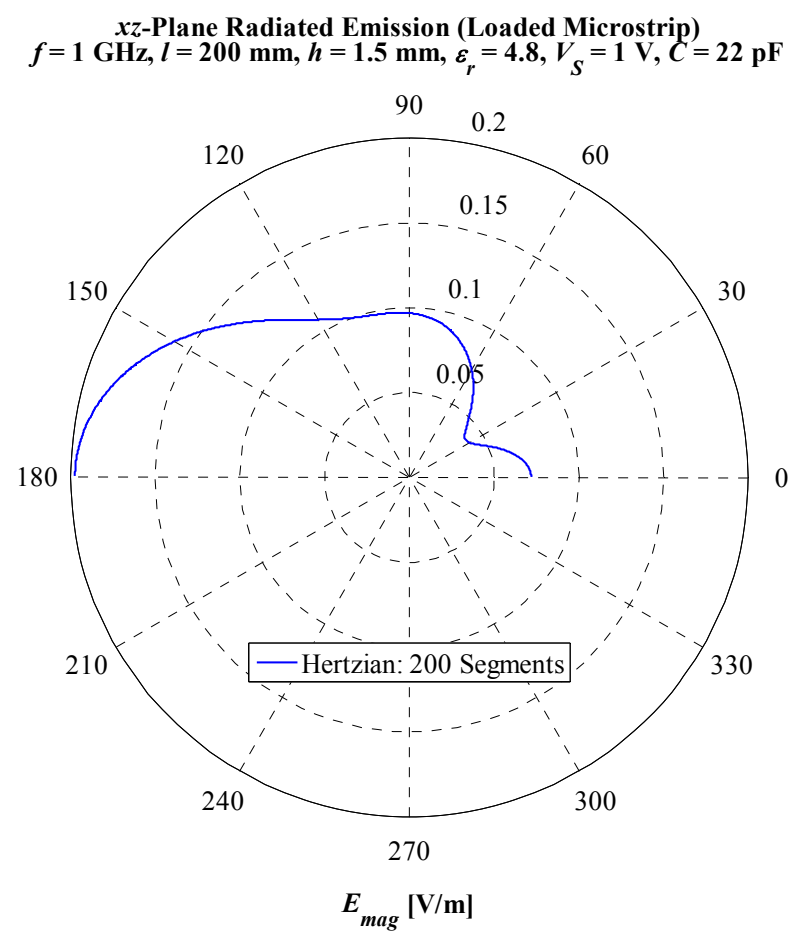

Figure 3.16 $x z$-plane observation with $22 \mathrm{pF}$ at load.

\subsection{Conclusion}

It has been shown, from a series of simulations, that indeed Schelkunoff's derivations is the same as integrating co-linear Hertzian dipoles, but assumes a free space wave number. Just like the Hertzian dipole it also shows the effects of discontinuities at the end points of the line if there is an abrupt change in the current (i.e. current loop is not closed). Because of these limitations the Hertzian model was used exclusively for investigating the microstrip. What is lacking in Schelkunoff's method is the ability to include dielectrics, as this requires $\beta_{l} \neq \beta_{\mathrm{o}}$ to be satisfied, and the inclusion of vertical dipoles to model polarisation effects. 


\section{Chapter 4. Experimental Results}

\subsection{Introduction}

Experimental validation of the models developed in the previous chapter is an important element of this thesis. In the physical domain things that can go wrong usually do go wrong (Murphy's Law!). Much attention and care have been devoted to obtaining good measurements of the electric field.

This chapter documents the measurement process beginning with a description of the semi-anechoic chamber, antenna, spectrum analyser and the microstrip.

The following key topics are covered:

1. Introduction to the test facility.

2. The test setup to provide sound and repeatable measurements for radiated fields from PCB structures on the chamber ground plane.

3. Validation of the model for TL currents (Chapter 2) and emissions (Chapter 3).

\subsection{Test Facility}

The radiated electric field measurement has to be conducted in a completely shielded chamber to remove external interference. Ferrite tiles and absorbers are used in the interior to prevent reflections from the walls and provide a "free space" environment. Such a facility is the semi-anechoic chamber available in Curtin University building 314. A map is given in Appendix D. The chamber is a FACT-3 Lingred with reduced dimensions.

\subsection{Test Measurement Setup}

The measurement setup is as shown in Figure 4.1. A close-up of the microstrip is shown in Figure 4.2. The tracking generator (TG) output from the receiver (Recv) was used to excite the microstrip. The electric field was measured in the vertical 
polarisation at a height of $1.5 \mathrm{~m}$. and a distance of $2.8 \mathrm{~m}$ from the load end (i.e. $3 \mathrm{~m}$ from the source end) of the microstrip.

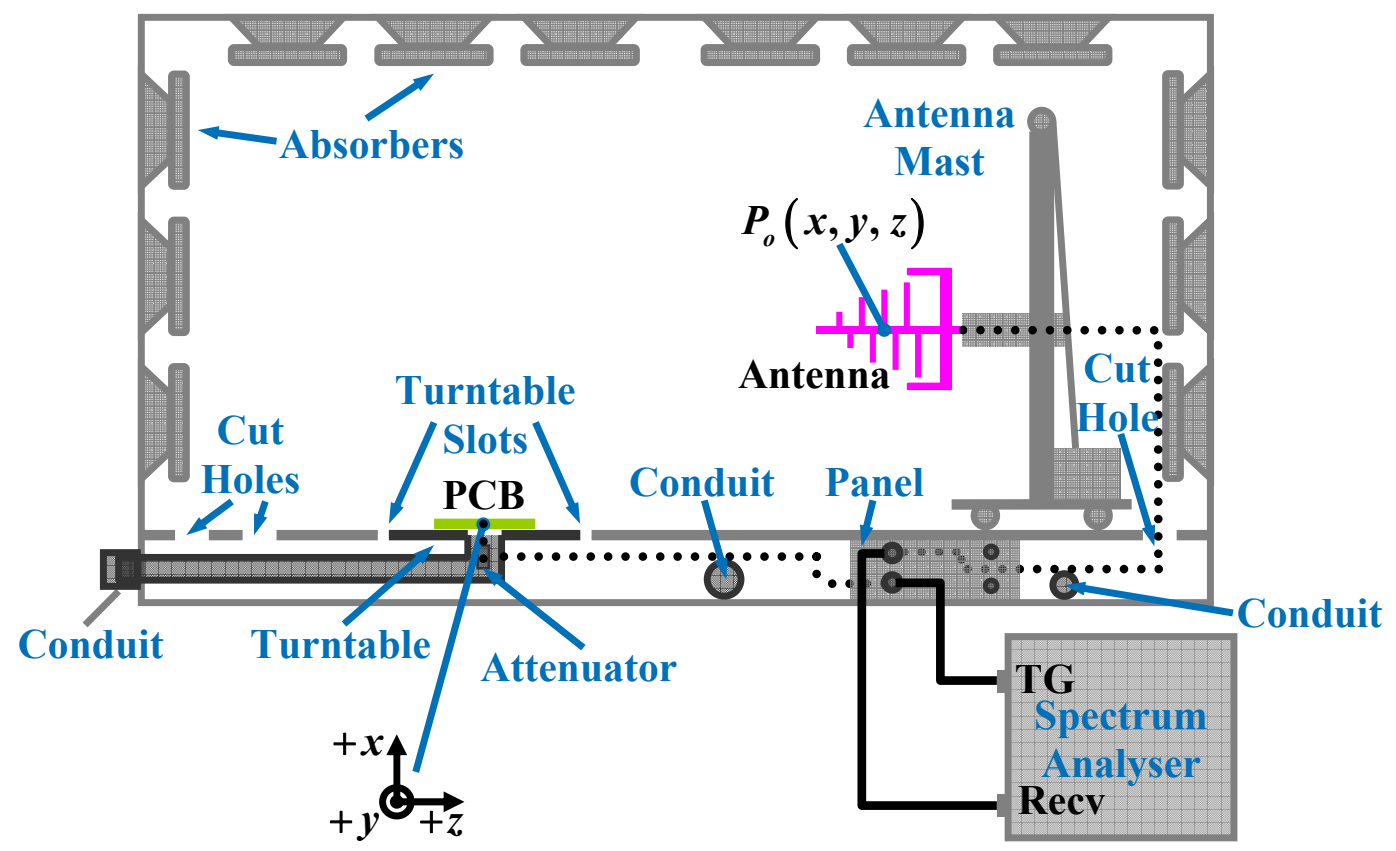

Figure 4.1 Test measurement setup.

\subsection{Measurement Procedure}

Validation of theory with electric field measurements, or vice-versa, is often difficult and requires care in the experimental setup. With measurements there is always some uncertainty related to imperfections in measurement equipment, the antenna factor calibration (i.e. proximity effects of the ferrite wall and ground plane on the antenna factor calibration), the absorbers, the cables, and the ground plane.

While most of these factors are difficult to quantify and to correct for, a considerable attempt has been made to improve the ground plane. A closed loop calibration was performed to remove the cable characteristics from the measurements. 


\subsubsection{Ground Plane Improvements}

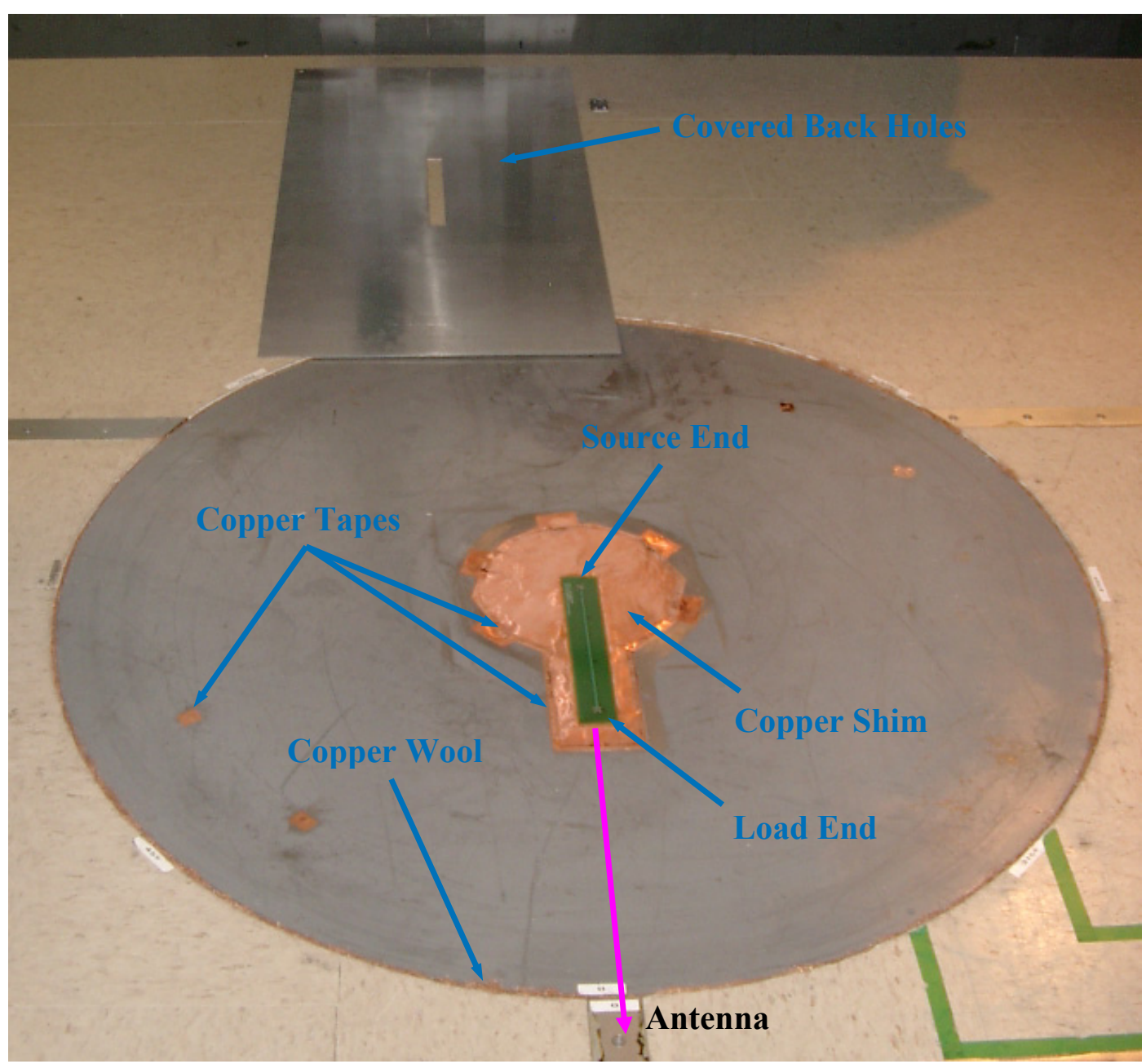

Figure 4.2 Chamber ground improvements.

For the measurements the ground of the microstrip structure was electrically connected to the chamber ground by means of copper tape and copper shim. The close proximity of the radiating device to the ground plane requires the ground to be of better integrity than what would be required if the device were elevated, as is typically done in compliance measurement. For this reason improvements were made to the chamber ground. These improvements included covering holes with copper tape and copper shim, a metal plate and by filling the gap around the turntable with copper wool. This is shown in Figure 4.2.

Ideally a perfect ground is required for these measurements as this assumption is foundational to the use of image currents as was done in the current and radiation model (AS5). Obviously, this is not possible in reality. In the actual test setup the 
microstrip is driven with a cable. If care is not taken common mode currents may exist along the cable and cause it to radiate, hence interfering with the actual emissions from the microstrip. A method to combat this is to ensure that the entire cable is below the ground plane. This is achieved by using a copper shim to connect the microstrip's ground to the chamber ground around the entire periphery of the PCB. This ensures that the trace is the dominant radiator in the chamber. As can be seen from Figure 4.2, all of the cables are under the chamber ground. The antenna should not see any cables at all, other than the one connected to it coming from the spectrum analyser.

\subsubsection{Loop and Antenna Calibration}

The loop calibration is an important part of the measurement procedure. This can be shown in Figure 4.3.

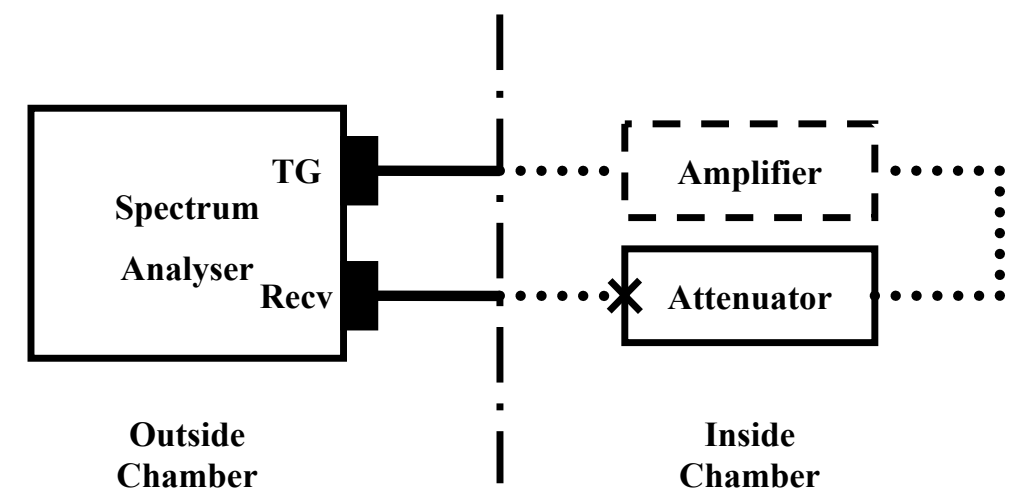

Figure 4.3 Loop calibration.

In the loop calibration the spectrum analyser is used to perform the frequency sweep measurement. The idea of having a loop calibration is to perform a measurement to capture characteristic of the cables, attenuator and an optional amplifier. Keeping the setup with the minimal number of cables and devices in the path is also very important. It is a good practice to use common mode ferrites on all the cables in the loop calibration and to remain in place for the actual microstrip emission measurement. As is seen in Figure 4.3 an attenuator is placed before the receiving end for two reasons, (i) so that excessive power does not arrive at the input to the receiver and (ii) to dampen any reflections arising from a mismatch between the 
microstrip and the cable that will interfere with the receiving end, whist still having enough dynamic range. From the illustration ' $x$ ' is the point where the loop and the tracking generator end is attached to the microstrip and the receiving end to the antenna. The loop calibration factor obtained in this way needs to be factored into the measurements together with the antenna factor provided by the manufacturer. The electric field measure is obtained as:

$$
E(\mathrm{~dB} \mu \mathrm{V} / \mathrm{m})=V(\mathrm{~dB} \mu \mathrm{V})+A F(\mathrm{~dB} / \mathrm{m})+C L(\mathrm{~dB}),
$$

where $A F$ is the antenna factor and $C L$ is the cable loss. This procedure has been shown to be very effective and give good repeatable results.

\subsection{Measurement Results}

In order to verify the models presented in Chapter 2 and 3, a microstrip of dimensions height: $h=1.5 \mathrm{~mm}$, trace width $w=2.5 \mathrm{~mm}$, length $l=204 \mathrm{~mm}$ and $\varepsilon_{r}=4.8$ was constructed (see Figure 4.2).

Four different terminations were investigated;

1. A terminated $\operatorname{load}\left(Z_{L}=51 \Omega\right)$;

2. A short circuit $\left(Z_{L}=0\right)$;

3. An open circuit $\left(Z_{L}=\infty\right)$; and

4. A capacitor load $\left(Z_{L}=22 \mathrm{pF}\right)$ with series inductance to represent the input impedance of an IC gate.

For each termination the input impedance was measured using a network analyser and compared to the impedance value obtained using the model for current in Chapter 2 with the voltage at the input of the line given by (Appendix A) 


$$
\begin{aligned}
Z_{\text {in }}(z) & =\frac{\hat{V}(z)}{\hat{I}(z)} \\
& =Z_{\mathrm{o}} \frac{Z_{L} \cosh \gamma\left(\frac{l}{2}-z\right)+Z_{\mathrm{o}} \sinh \gamma\left(\frac{l}{2}-z\right)}{Z_{\mathrm{o}} \cosh \gamma\left(\frac{l}{2}-z\right)+Z_{L} \sinh \gamma\left(\frac{l}{2}-z\right)} .
\end{aligned}
$$

At the boundary $z=-l / 2$ at the input port:

$$
Z_{\text {in }}\left(-\frac{l}{2}\right)=Z_{\mathrm{o}} \frac{Z_{L} \cosh \gamma l+Z_{\mathrm{o}} \sinh \gamma l}{Z_{\mathrm{o}} \cosh \gamma l+Z_{L} \sinh \gamma l} Z_{\mathrm{o}} .
$$

The electric field emission was measured and compared to that obtained using the Hertzian method given in Chapter 3 for 200 segments.

\subsubsection{Matched Load}

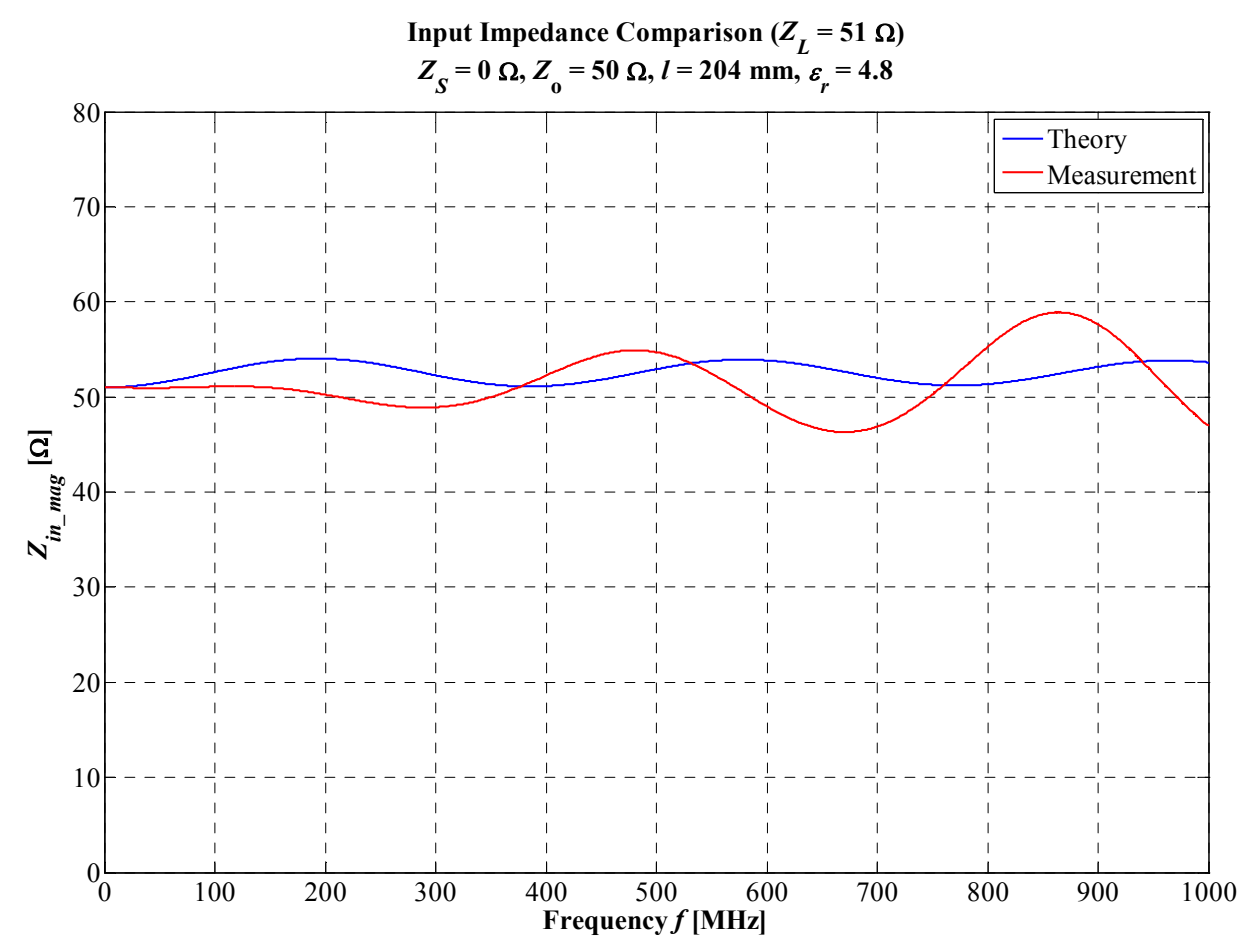

Figure 4.4 Input impedance comparison for $51 \Omega$. 


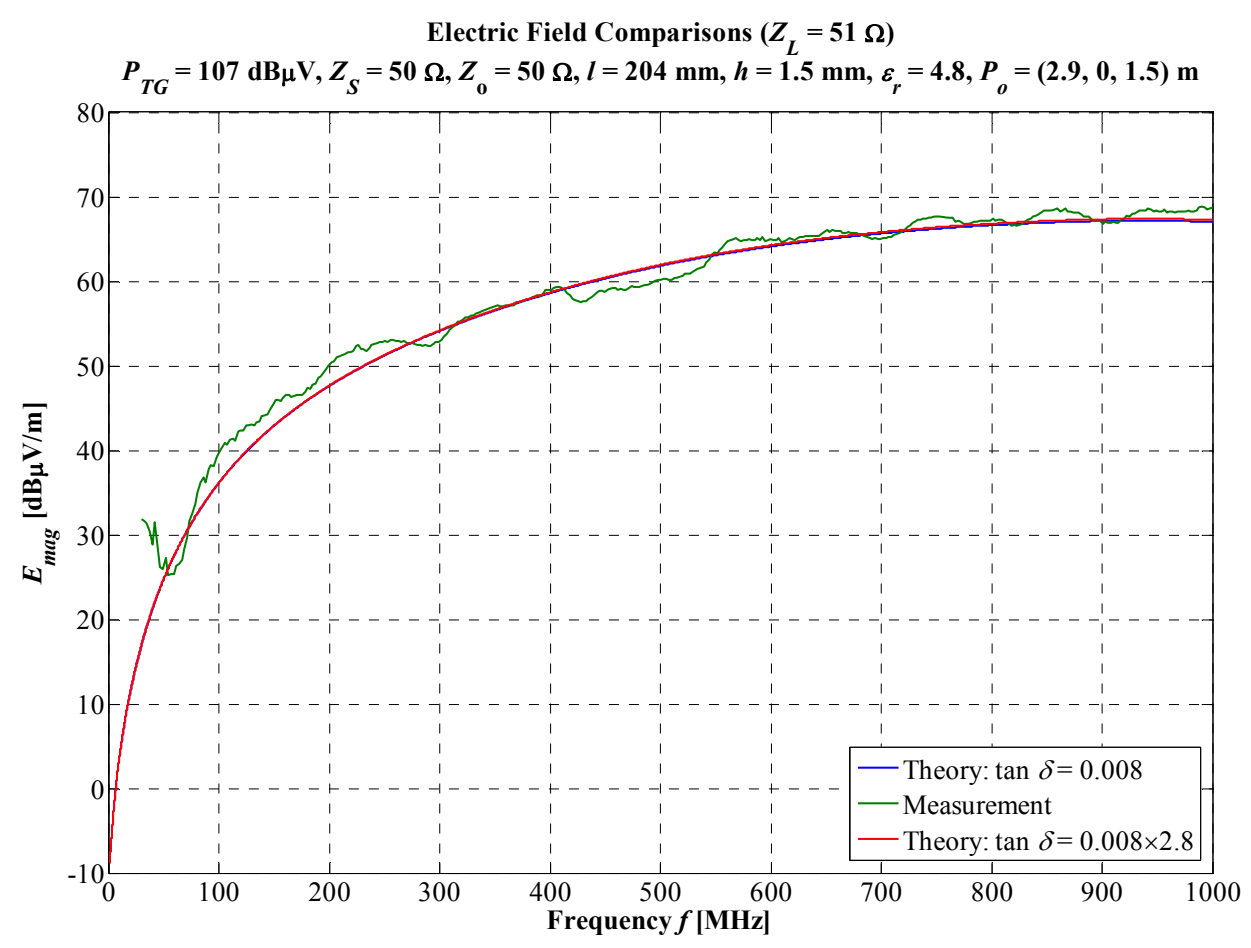

Figure 4.5 Electric field comparison for $51 \Omega$.

\subsubsection{Short}

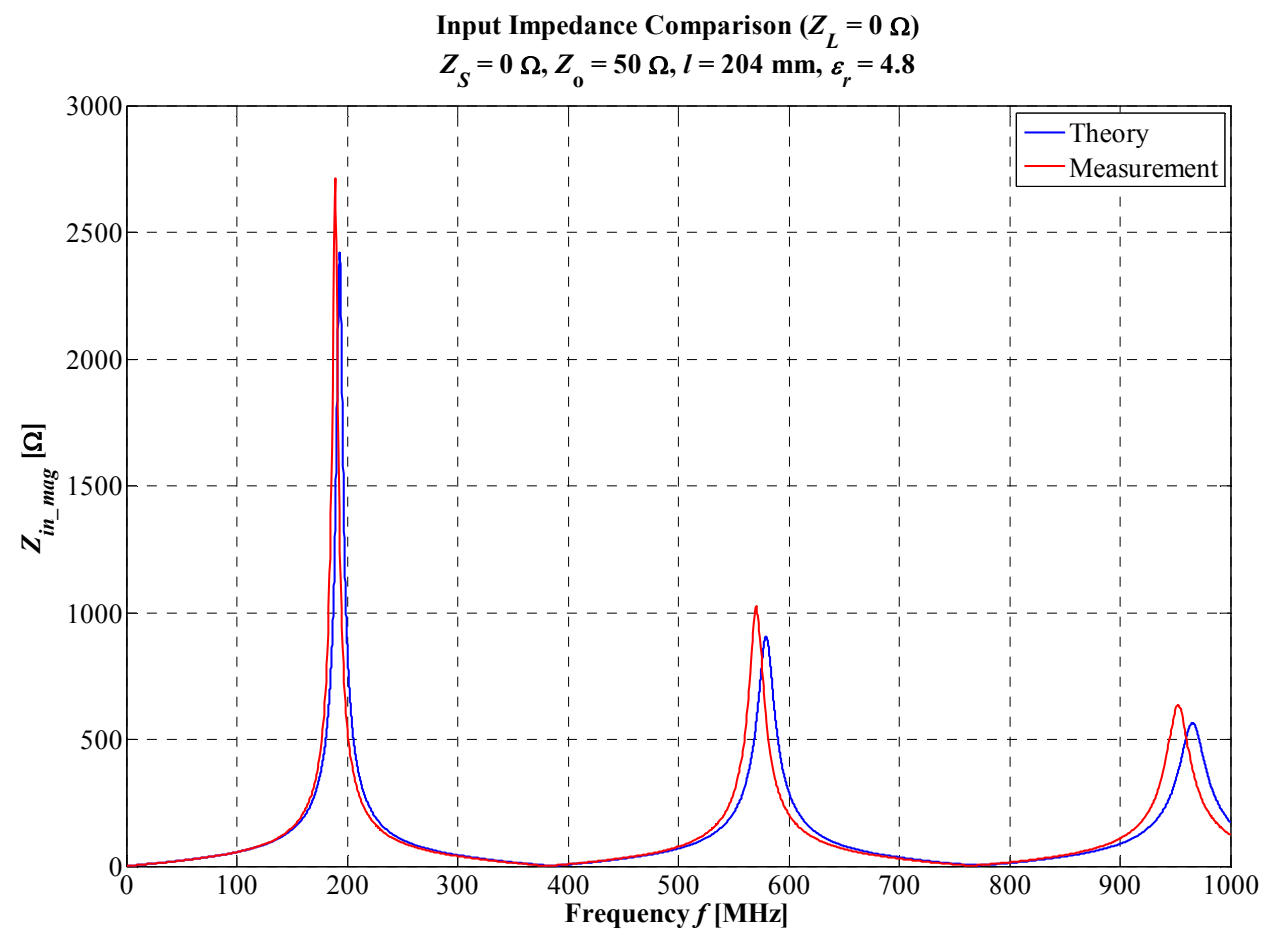

Figure 4.6 Input impedance comparison for short. 


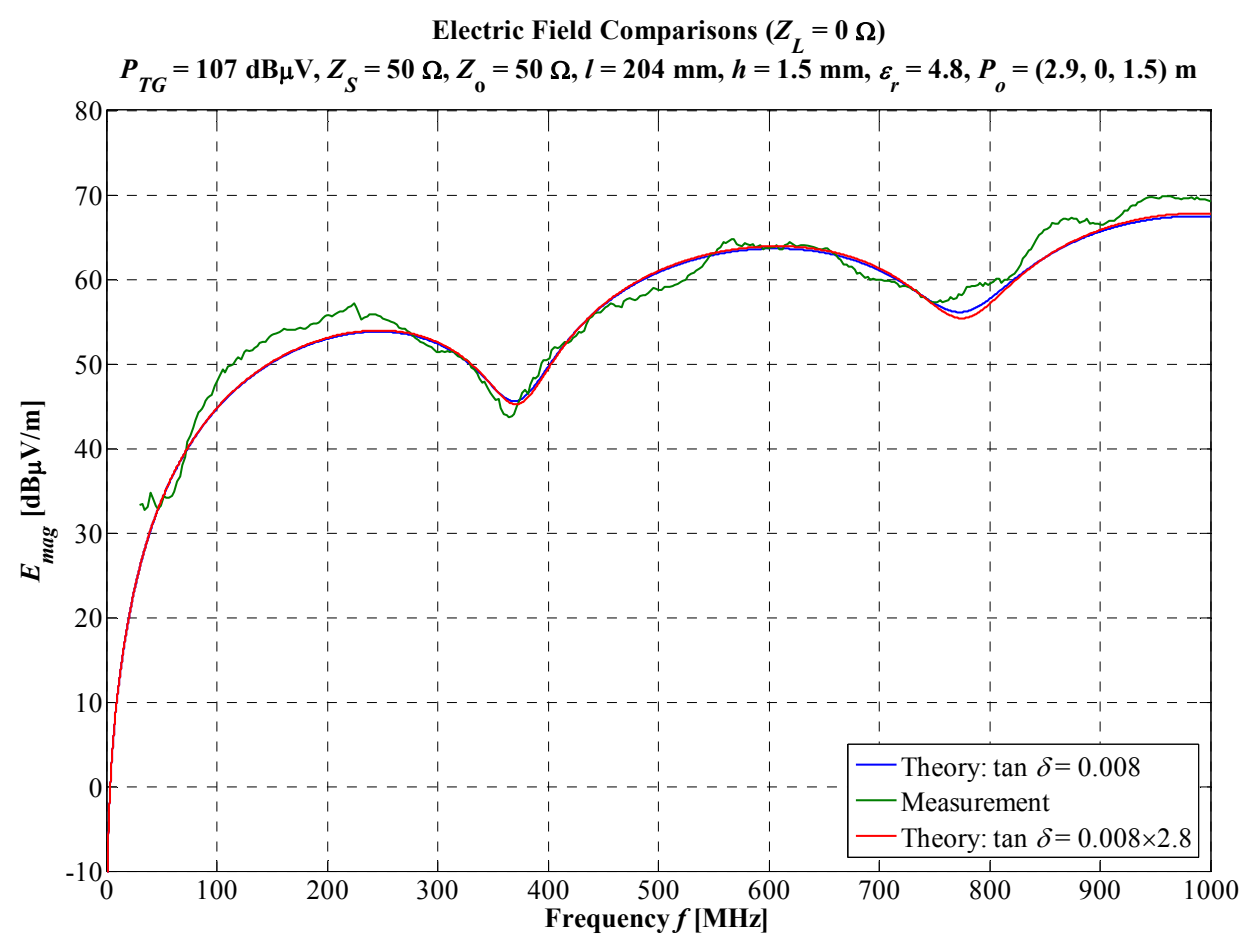

Figure 4.7 Electric field comparison for short.

\subsubsection{Open}

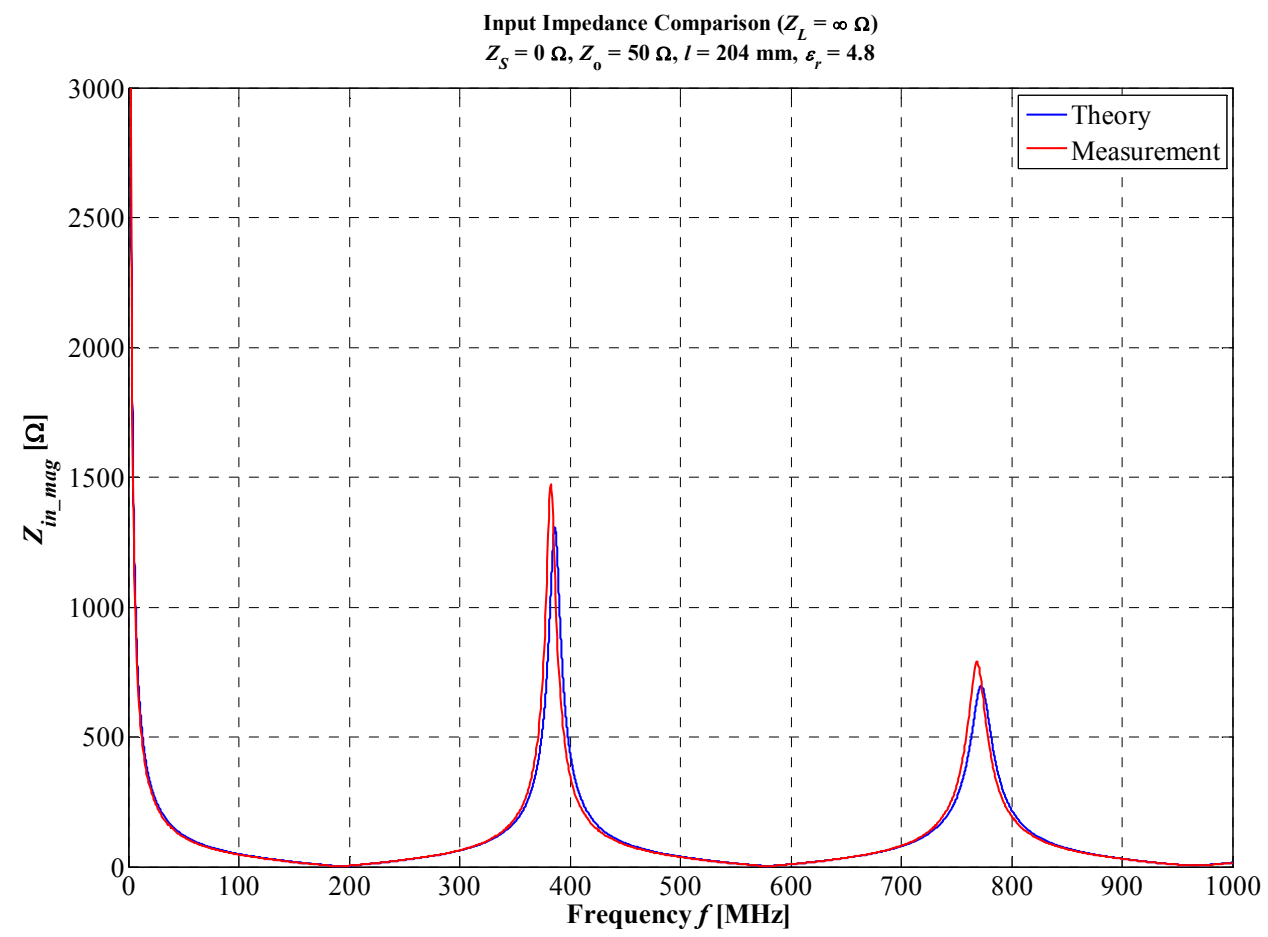

Figure 4.8 Input impedance comparison for open. 


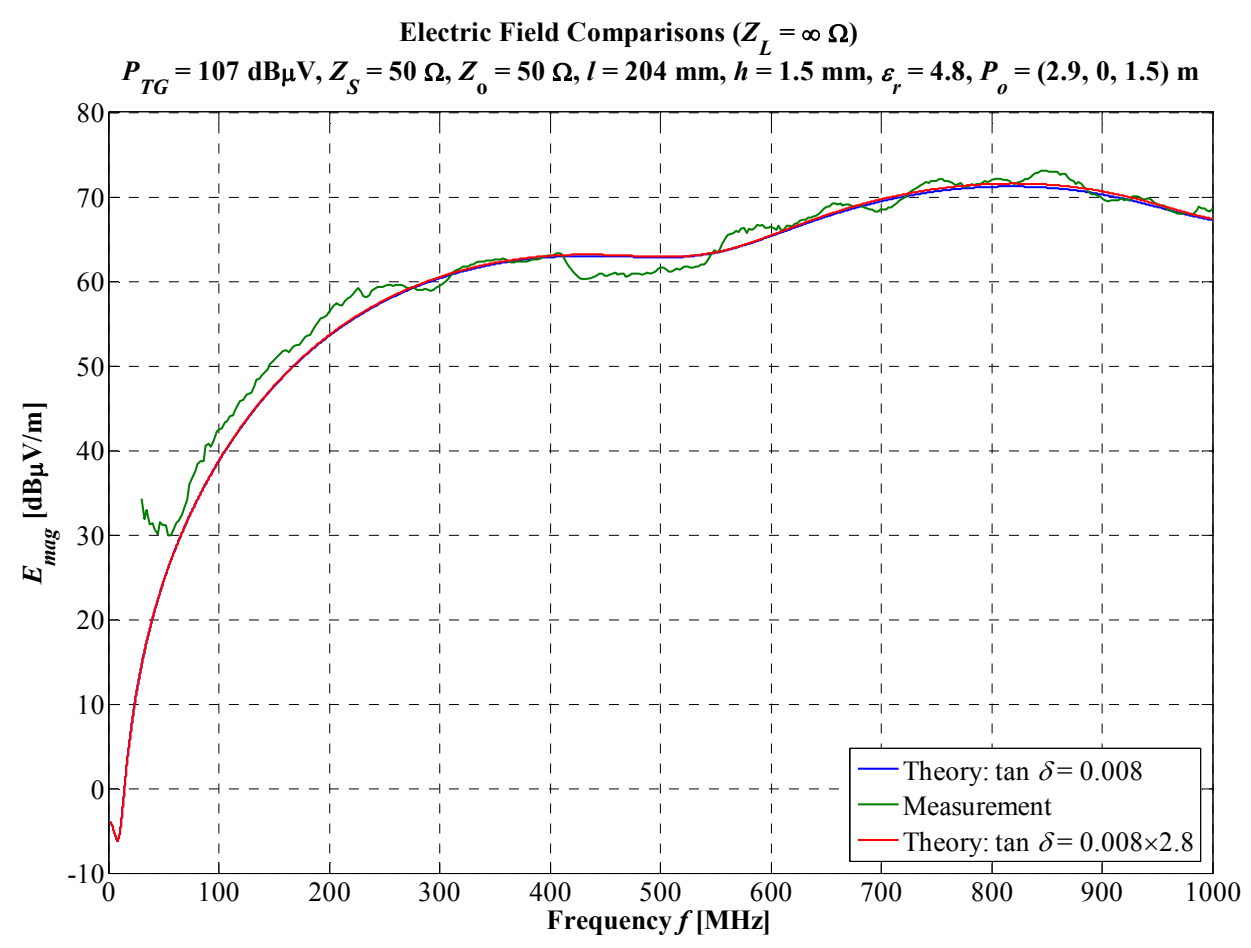

Figure 4.9 Electric field comparison for open.

\subsubsection{2 pF Capacitor}

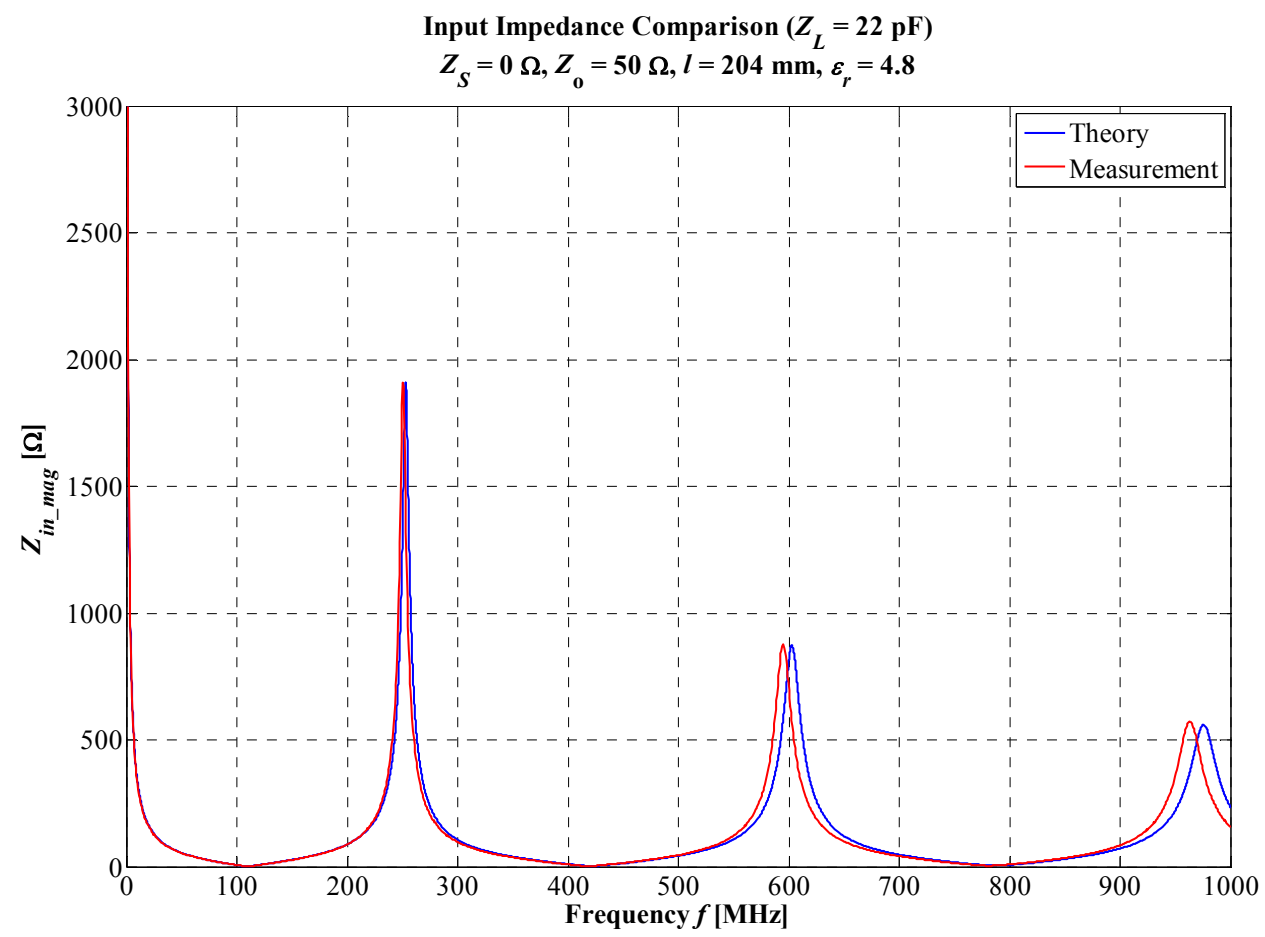

Figure 4.10 Input impedance comparison for $22 \mathrm{pF}$ capacitor. 


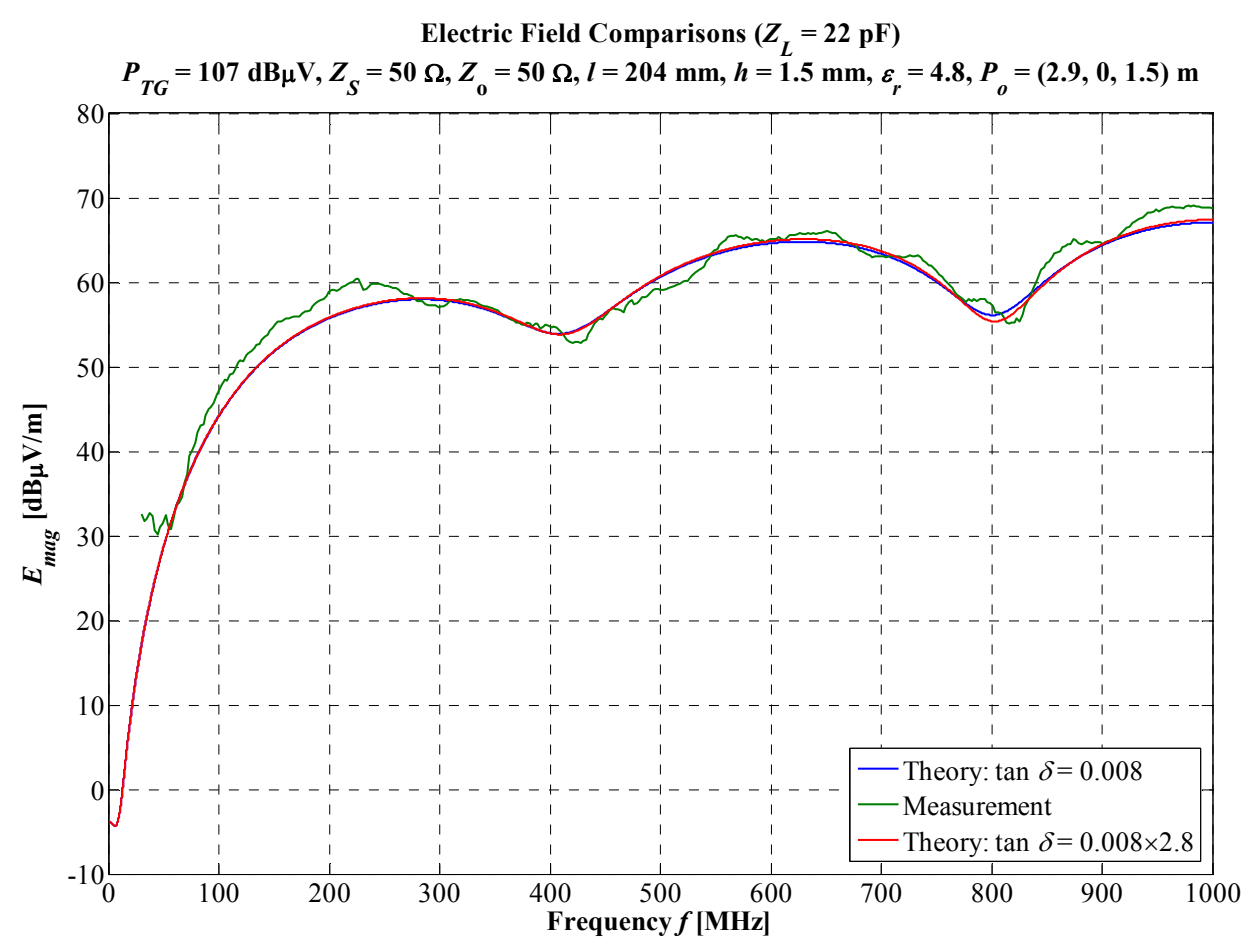

Figure 4.11 Electric field comparison for $22 \mathrm{pF}$ capacitor.

Note that a series inductance of $0.5 \mathrm{nH}$ has been added to the $Z_{L}=22 \mathrm{pF}$ load in the analytical model to represent a realistic capacitor.

\subsection{Conclusion}

The loss tangent $\tan \delta$ was artificially increased by the value $\tan \delta=0.008 \times 2.8$ to make the peaks 'match' for the input impedance. The loss tangent variations of the PCB under investigation are subjected to manufacturing process variations and so affect the loss tangent of a FR-4 dielectric material of around 0.008. Other reasons may be due to the physical constriction of the microstrip and modifications to the PCB necessary to perform these measurements. More likely, the need for the artificial factor is due to the radiation loss that has been ignored. It is interesting, however, that in terms of the radiation the artificial factor of 2.8 has little effect. This can be seen from the plots of the test cases given in Figure 4.5, Figure 4.7, Figure 4.9 and Figure 4.11. These results justify assumptions AS by demonstrating that radiation losses can indeed be ignored in the modelling of TL currents for the purpose of obtaining field emissions. 
Very good agreement has been obtained between measurement and the models developed in Chapters 2 and 3. This provides confidence to use the radiation model for source series damping and destination parallel damping analysis in the next chapter. 


\section{Chapter 5. Source and Destination Damping of TLs}

\subsection{Introduction}

In previous chapters the development of an emission model using the Hertzian dipole method was described and verified with measurements. Leading up to this point considerable attention was given to the modelling of the TL, polarisation currents and electric field computation. These were necessary to show that by using TL theory, in conjunction with Wheeler's equations for a microstrip, the Hertzian dipole technique is able to predict radiated emissions that agree well with the measurement given in Chapter 4. Having obtained confidence in the microstrip radiation model through validation by measurement, it is possible, in this chapter to address the impact of terminations on radiation. This chapter compares the emissions from the source damping and destination damping topologies. Comparisons are made for these two topologies that have the same damping factor and voltage at the load end. Under these conditions the source and destination damping have a similar behaviour as far as signal integrity is concerned.

\subsection{Damping}

Consider the source series damping (ssd) and destination parallel damping (dpd) arrangement in Figure 5.1.

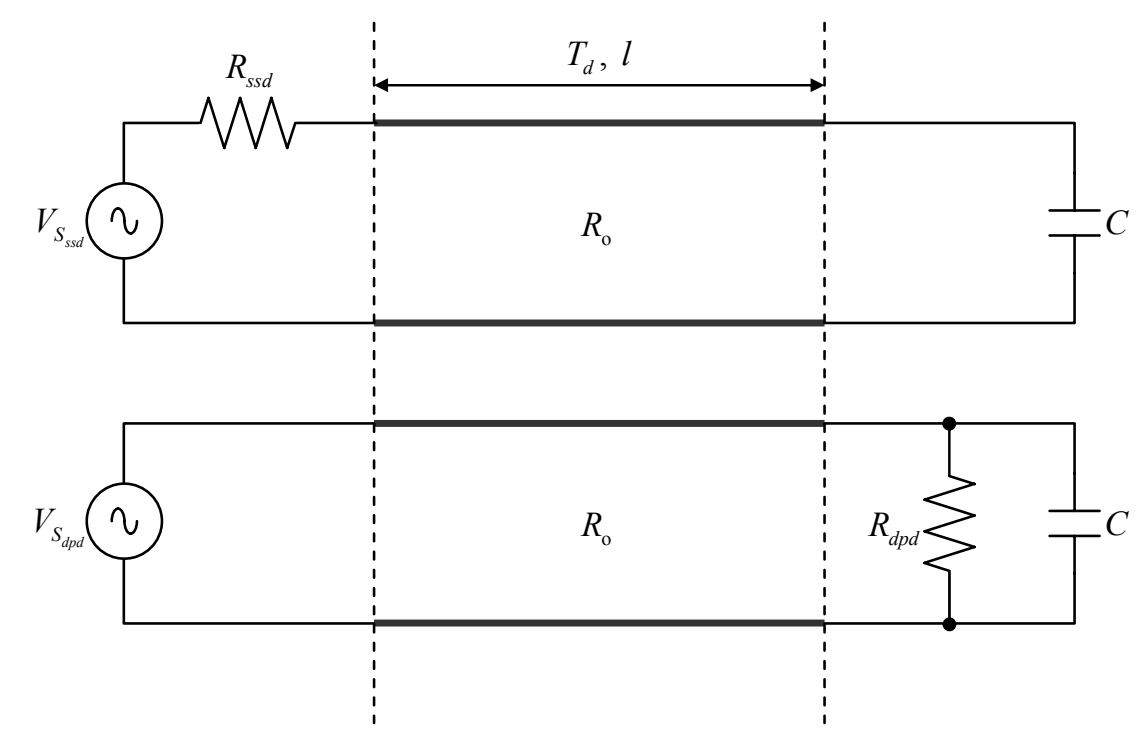

Figure 5.1 Top: Source series damping, Bottom: Destination parallel damping. 
For a capacitive load, $C$, connected to the end of a TL the frequency dependent damping factors $\zeta_{\text {ssd }}(\omega)$ at the source end and $\zeta_{d p d}(\omega)$ at the destination end, to a first order approximation, are given by [33]:

$$
\begin{gathered}
\zeta_{s s d}(\omega)=\frac{1}{2} \frac{R_{s s d}+R_{\mathrm{o}} \tanh (l \cdot \alpha(\omega))}{R_{\mathrm{o}}+R_{s s d} \tanh (l \cdot \alpha(\omega))} \frac{R_{\mathrm{o}} C+T_{d}}{\sqrt{R_{\mathrm{o}} C T_{d}}}, \text { and } \\
\zeta_{d p d}(\omega)=\frac{1}{2} \frac{T_{d} R_{\mathrm{o}}+\left(R_{\mathrm{o}} C+T_{d}\right) R_{d p d} \tanh (l \cdot \alpha(\omega))}{R_{d p d}+R_{\mathrm{o}} \tanh (l \cdot \alpha(\omega))} \sqrt{\frac{R_{d p d}+R_{\mathrm{o}} \tanh (l \cdot \alpha(\omega))}{R_{d p d} R_{\mathrm{o}} C T_{d}}},
\end{gathered}
$$

where $\alpha(\omega)$ is the loss component of the propagation constant and $T_{d}$ is the time delay of the transmission line:

$$
T_{d}=\frac{l}{u}
$$

where $l$ is the length of the line and $u$ is the velocity of propagation. Resistors $R_{s s d}$ and $R_{d p d}$ are as shown in Figure 5.1.

The natural frequency for the source damping case of a line of characteristic resistance $\left(R_{\mathrm{o}}=\operatorname{Re}\left\{Z_{\mathrm{o}}\right\}\right)$, time delay $T_{d}$ and load capacitance $C$ is [21,33]

$$
\omega_{\mathrm{o}_{s s d}}=\frac{1}{\sqrt{R_{\mathrm{o}} C T_{d}}} .
$$

For the parallel damping the natural frequency is a function of $R_{d p d}, l$ and $\alpha(\omega)$ as well

$$
\omega_{\mathrm{o}_{d p d}}=\sqrt{\frac{R_{d p d}+R_{\mathrm{o}} \tanh \left(l \cdot \alpha\left(\omega_{\mathrm{o}_{d p d}}\right)\right)}{R_{d p d} R_{\mathrm{o}} C T_{d}}} .
$$

Since $R_{\mathrm{o}} \tanh \left(l \cdot \alpha\left(\omega_{\mathrm{o}_{\text {dpd }}}\right)\right) \ll R_{d p d}$ and to a first order approximation

$$
\tanh \left(l \cdot \alpha\left(\omega_{\mathrm{o}_{d p d}}\right)\right) \approx \tanh \left(l \cdot \alpha\left(\omega_{\mathrm{o}_{s s d}}\right)\right)
$$


giving

$$
\omega_{\mathrm{o}_{d p d}} \approx \sqrt{\frac{R_{d p d}+R_{\mathrm{o}} \tanh \left(l \cdot \alpha\left(\omega_{\mathrm{o}_{s s d}}\right)\right)}{R_{d p d} R_{\mathrm{o}} C T_{d}}} .
$$

The source series damping resistance, can be obtained as [33]

$$
R_{s s d}=\frac{R_{\mathrm{o}}\left(2 d \zeta_{s s d}-\tanh \left(l \cdot \alpha\left(\omega_{\mathrm{o}_{s s d}}\right)\right)\right)}{1-2 d \zeta_{s s d} \tanh \left(l \cdot \alpha\left(\omega_{\mathrm{o}_{s s d}}\right)\right)}
$$

with

$$
d=\frac{\sqrt{R_{\mathrm{o}} C T_{d}}}{R_{\mathrm{o}} C+T_{d}} .
$$

which must satisfy the following bound

$$
\tanh \left(l \cdot \alpha\left(\omega_{\mathrm{ossd}}\right)\right) \leq \zeta_{s s d}<\frac{1}{\tanh \left(l \cdot \alpha\left(\omega_{\mathrm{o}_{s d d}}\right)\right)}
$$

For the typical trace dimensions this lower bound is small and roughly in the order $10^{-2}$ and upper bound less than $10^{1}$, so realistic values of damping would normally be within this range.

For the case of the destination damping the value of $R_{d p d}$ to calculate damping at $\omega_{\mathrm{o}_{d p d}}$ is [33]

$$
\begin{gathered}
R_{d p d}=\frac{-b_{\omega_{\text {odpd }}}+\sqrt{b_{\omega_{\text {odpd }}}^{2}-4 a_{\omega_{\text {odpd }}} c}}{2 a_{\omega_{\text {odpd }}}} \\
a_{\omega_{\text {odpd }}}=4 R_{\mathrm{o}} C T_{d}\left(\zeta_{d p d}\left(\omega_{\mathrm{o}_{\text {dpd }}}\right)\right)^{2}-\left(R_{\mathrm{o}} C+T_{d}\right)^{2} \tanh ^{2}\left(l \cdot \alpha\left(\omega_{\mathrm{o}_{d p d}}\right)\right),
\end{gathered}
$$




$$
\begin{gathered}
\left(2 R_{\mathrm{o}} T_{d} \tanh \left(l \cdot \alpha\left(\omega_{\mathrm{d}_{d p d}}\right)\right)\right) \\
b_{\omega_{\text {odpd }}}=\left(2 R_{\mathrm{o}} C\left(\zeta_{d p d}\left(\omega_{\mathrm{o}_{d p d}}\right)\right)^{2}-\left(R_{\mathrm{o}} C+T_{d}\right) \tanh \left(l \cdot \alpha\left(\omega_{\mathrm{o}_{d p d}}\right)\right)\right)^{\prime} \\
c=-R_{\mathrm{o}}^{2} T_{d}^{2}
\end{gathered}
$$

which must satisfy

$$
\frac{\left(R_{\mathrm{o}} C+T_{d}\right) \tanh \left(l \cdot \alpha\left(\omega_{\mathrm{o}_{\text {dpd }}}\right)\right)}{2 \sqrt{R_{\mathrm{o}} C T_{d}}} \leq \zeta_{d p d}<\infty .
$$

For the typical trace dimensions this lower bound is small and roughly in the order $10^{-2}$.

The damping equations presented thus far are too complicated to quickly extract dominant asymptotic behavioural parameters. By further assuming $R_{\mathrm{o}} C_{L} \gg T_{d}$ and $\alpha=0$ the damping factors for low frequencies are approximately:

$$
\begin{gathered}
\zeta_{s s d}=\frac{1}{2} \frac{R_{s s d}}{R_{\mathrm{o}}} \sqrt{\frac{R_{\mathrm{o}} C}{T_{d}}}, \\
\zeta_{d p d}=\frac{1}{2} \frac{R_{\mathrm{o}}}{R_{d p d}} \sqrt{\frac{T_{d}}{R_{\mathrm{o}} C}},
\end{gathered}
$$

and damping resistances are

$$
\begin{gathered}
R_{s s d}=2 R_{\mathrm{o}} \sqrt{\frac{T_{d}}{R_{\mathrm{o}} C}} \zeta_{s s d}, \\
R_{d p d}=\frac{R_{\mathrm{o}}}{2 \zeta_{d p d}} \sqrt{\frac{T_{d}}{R_{\mathrm{o}} C}} .
\end{gathered}
$$


The different damping values the damping type can be classified according to Table 5.1 .

\begin{tabular}{|c|c|}
\hline Damping & Type \\
\hline \hline$\zeta=0$ & Undamped \\
\hline $0<\zeta<1$ & Underdamped \\
\hline$\zeta=1$ & Critically Damped \\
\hline$\zeta>1$ & Overdamped \\
\hline
\end{tabular}

Table 5.1 Damping chracteristics.

In order to compare emissions under source and destination damping, it is required that the damping be the same. Thus $R_{s s d}$ given by equation (5.7) and $R_{d p d}$ given by equation (5.10.1) are calculated according to their full expressions for one desired value of damping at $\omega_{\mathrm{o}_{d p d}}=\omega_{\mathrm{o}_{s s}}$. These values must lie within the bounds specified in equations (5.9) and (5.11).

\subsection{Load Voltage}

For the comparisons the load voltage must be kept the same as mentioned in the introduction. This can be done by assuming a fixed source voltage for the source damping case. The source voltage for the destination damping case must then be scaled appropriately to produce the same load voltage. As given in Appendix A the voltage at the load for source damping is:

$$
\begin{aligned}
\hat{V}_{L_{s s d}}(\omega) & =\frac{Z_{\mathrm{o}} \cdot Z_{L}(\omega)}{D_{s s d}(\omega)} \cdot \hat{V}_{S_{s s d}}(\omega) \\
& =A_{s s d}(\omega) \cdot \hat{V}_{S_{s s d}}(\omega)
\end{aligned}
$$

with

$$
A_{s s d}(\omega)=\frac{Z_{\mathrm{o}} \cdot Z_{L}(\omega)}{D_{s s d}(\omega)},
$$




$$
\begin{gathered}
D_{s s d}(\omega)=\left(\begin{array}{c}
R_{\mathrm{o}}\left(R_{s s d}+Z_{L_{s s d}}(\omega)\right) \cosh (l \cdot \gamma(\omega)) \\
+\left(R_{\mathrm{o}}^{2}+R_{s s d} Z_{L_{s s d}}(\omega)\right) \sinh (l \cdot \gamma(\omega))
\end{array}\right), \\
Z_{L_{s s d}}(\omega)=j\left(X_{L_{C}}(\omega)-X_{C}(\omega)\right),
\end{gathered}
$$

for the source damping case and

$$
\begin{aligned}
\widehat{V}_{L_{d p d}}(\omega) & =\frac{Z_{\mathrm{o}} \cdot Z_{L}(\omega)}{D_{d p d}(\omega)} \cdot \widehat{V}_{S_{d p d}}(\omega), \\
& =A_{d p d}(\omega) \cdot \widehat{V}_{S_{d p d}}(\omega)
\end{aligned}
$$

with

$$
\begin{gathered}
A_{d p d}(\omega)=\frac{Z_{\mathrm{o}} \cdot Z_{L}(\omega)}{D_{d p d}(\omega)}, \\
D_{s s d}(\omega)=\left(\begin{array}{l}
R_{\mathrm{o}} \cdot Z_{L_{d p d}}(\omega) \cdot \cosh (l \cdot \gamma(\omega)) \\
+R_{\mathrm{o}}^{2} \sinh (l \cdot \gamma(\omega))
\end{array}\right), \\
Z_{L_{s s d}}(\omega)=R_{d p d} \| j\left(X_{L_{C}}(\omega)-X_{C}(\omega)\right),
\end{gathered}
$$

for the destination damping case where

$$
\begin{gathered}
X_{C}(\omega)=\frac{1}{\omega C}, \\
X_{L_{C}}(\omega)=\omega L_{C},
\end{gathered}
$$

are the reactances of capacitor $C$, and its associated series or load inductance $L_{C}$.

Hence by equating equations (5.16.1) and (5.19.1) the source voltage for the destination damping case is:

$$
\widehat{V}_{S_{d p d}}(\omega)=\frac{A_{s s d}(\omega)}{A_{d p d}(\omega)} \cdot \widehat{V}_{S_{s s d}}(\omega) \text {. }
$$




\subsection{Results}

In this section various comparative emission plots are given for the source series damping and destination parallel damping case for different damping factors. A small amount of series inductance defined as $L_{C}$ of value $0.5 \mathrm{nH}$ was added to the capacitor to simulate parasitic inductances in the capacitor and associated connection to the TL. The effect of this inductance is that the two lines may no longer have the same damping, as the formulas (5.1.1) and (5.1.2) do not account for this inductance.

In Figure 5.2, Figure 5.3 and Figure 5.4 at the various observation points, $P_{o}$, the damping factors $($ Underdamped $=0.2$, Critically Damped $=1$, Overdamped $=4)$ are varied for each source and destination damping test set. From the plots it is quite obvious that there are to be three set of curves (i.e. three for the source damping case and three for the destination damping case). Similarly to Chapter 4 these sets of simulations are performed for a capacitance of $22 \mathrm{pF}$ and its series inductance of 0.5 $\mathrm{nH}$ with the same simulation parameters for the material properties, geometry and microstrip orientation. The only difference is that the source damping case is used as a reference for comparing with destination damping, and is driven by a constant voltage source of $\hat{V}_{S}=\hat{V}_{S_{s s d}}=1 \mathrm{~V}$. In Figure 5.5 shows the simulation for a different characteristic impedance by narrowing the trace width from $2.5 \mathrm{~mm}$ to $0.5 \mathrm{~mm}$ to give an effective characteristic impedance $Z_{o_{\text {eff }}} \simeq 106.30 \Omega$. All other parameters are unchanged and can be found from the plots in Chapter 4. In Figure 5.6, for critical damping, a comparison between source damping and destination damping is made for two values of $L_{C}(0.5 \mathrm{nH}$ and at $5 \mathrm{nH})$. 


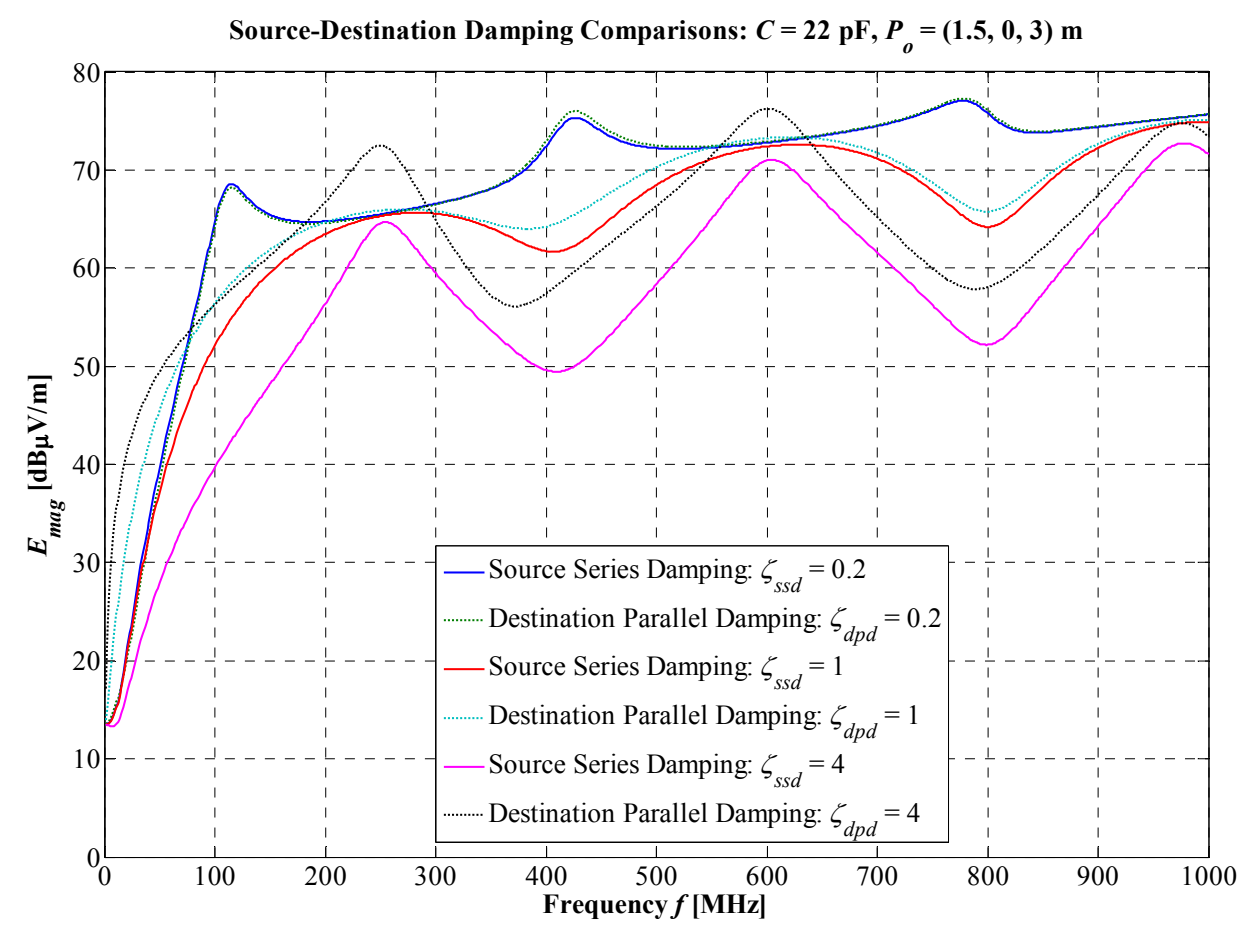

Figure 5.2 Effect of various damping at $P_{o}=(1.5,0,3) \mathrm{m}$ with

$$
Z_{o_{\text {eff }}} \simeq 52.53 \Omega \text {. }
$$

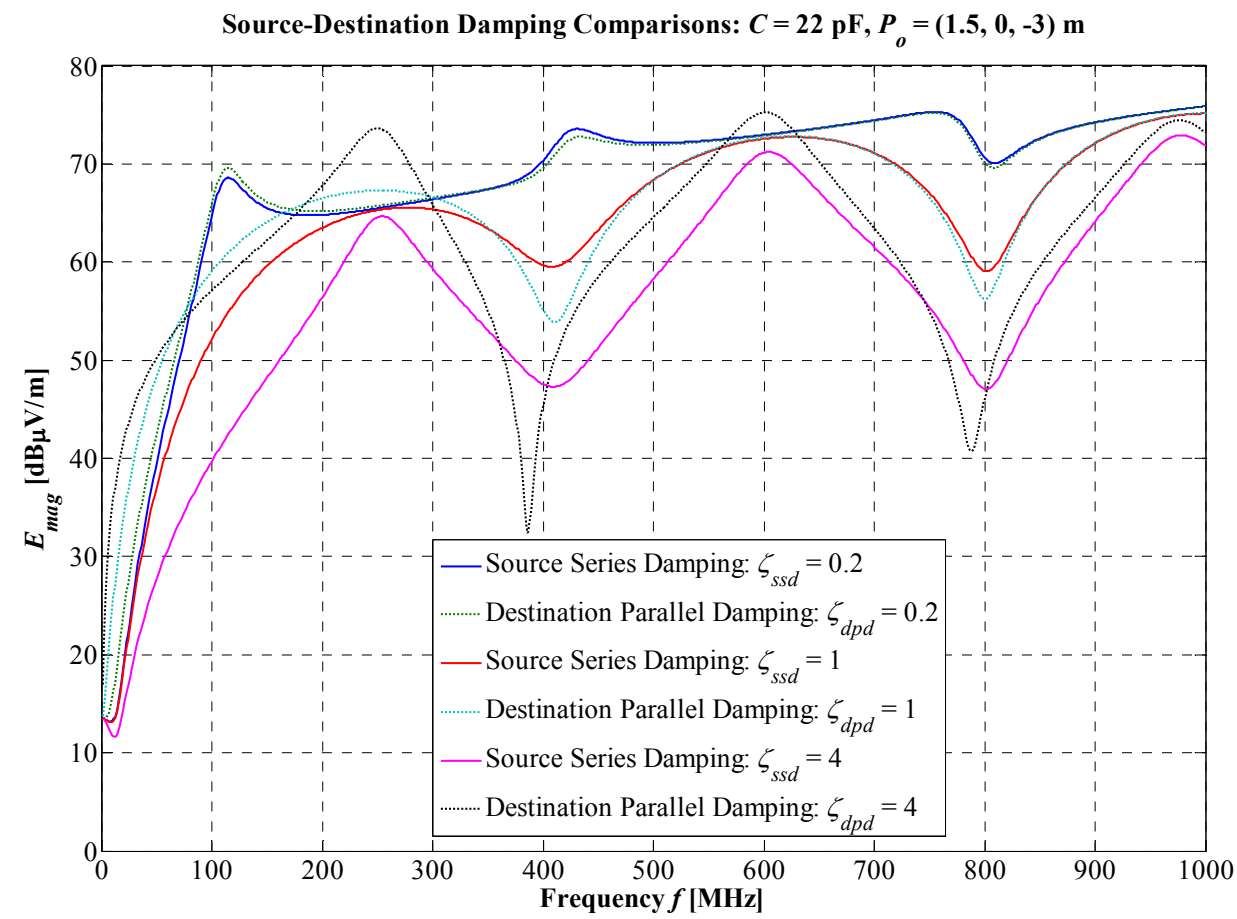

Figure 5.3 Effect of various damping at $P_{o}=(1.5,0,-3) \mathrm{m}$ with

$$
Z_{o_{\text {eff }}} \simeq 52.53 \Omega \text {. }
$$




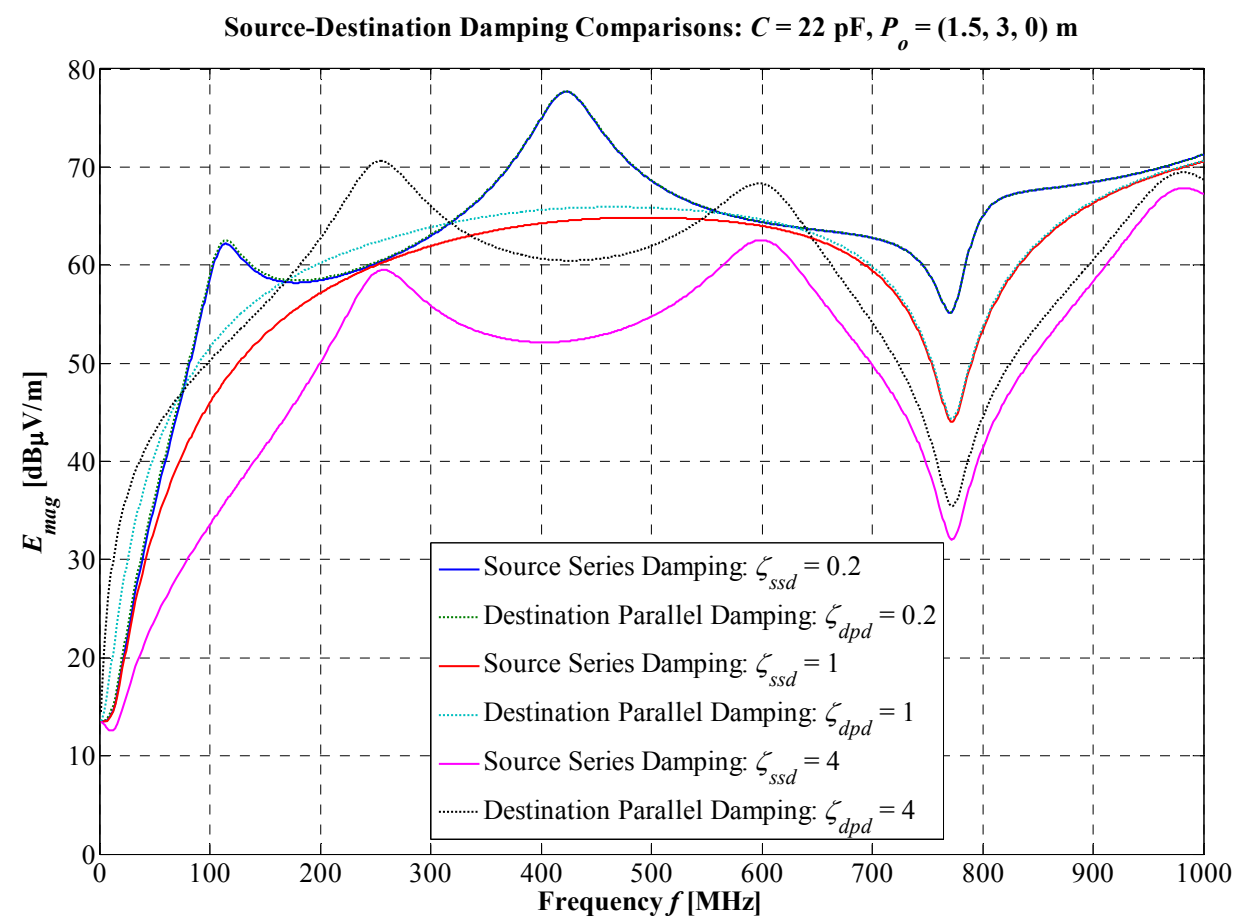

Figure 5.4 Effect of various damping at $P_{o}=(1.5,3,0) \mathrm{m}$ with

$$
Z_{o_{\text {eff }}} \simeq 52.53 \Omega \text {. }
$$

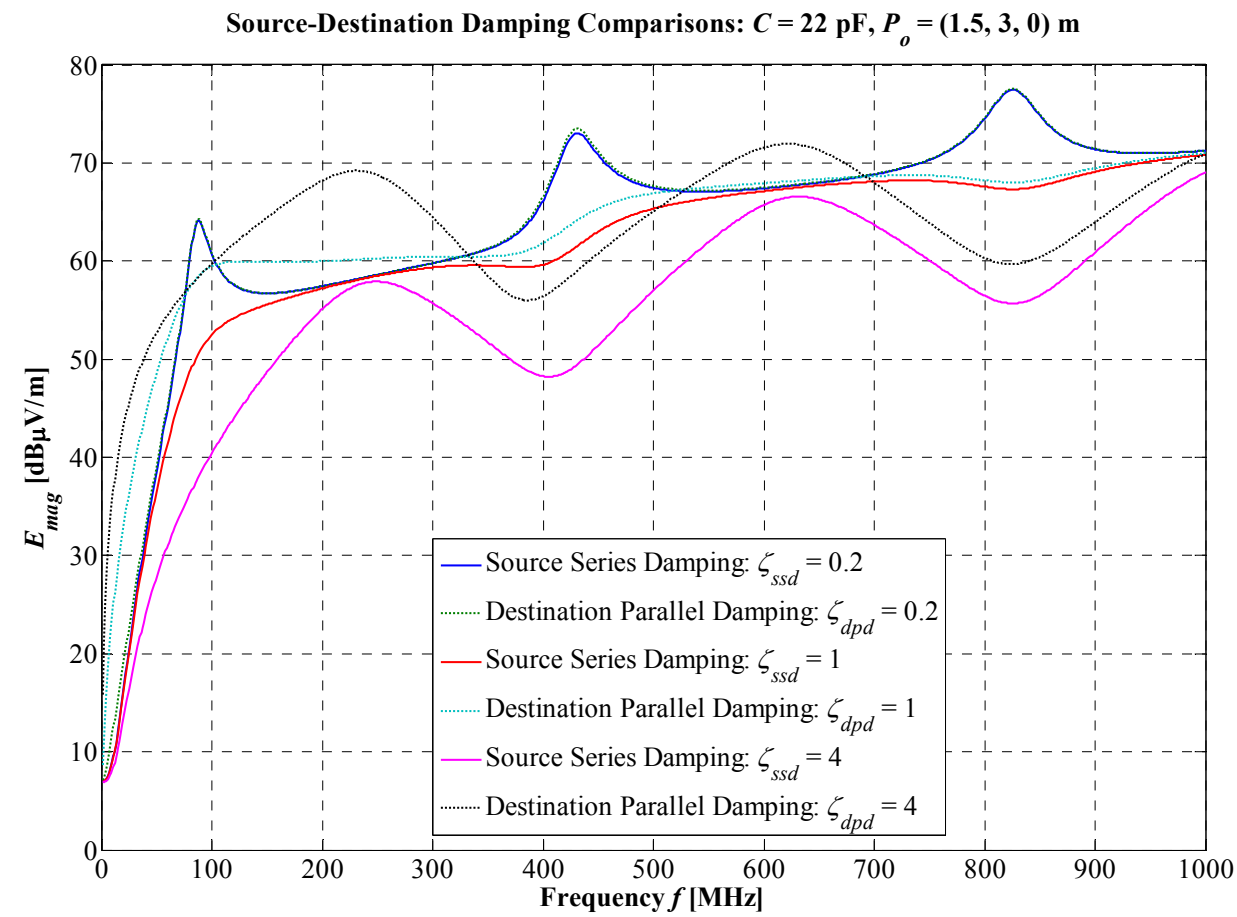

Figure 5.5 Effect of various damping at $P_{o}=(1.5,3,0) \mathrm{m}$ with

$$
Z_{o_{\text {eff }}} \simeq 106.30 \Omega \text {. }
$$




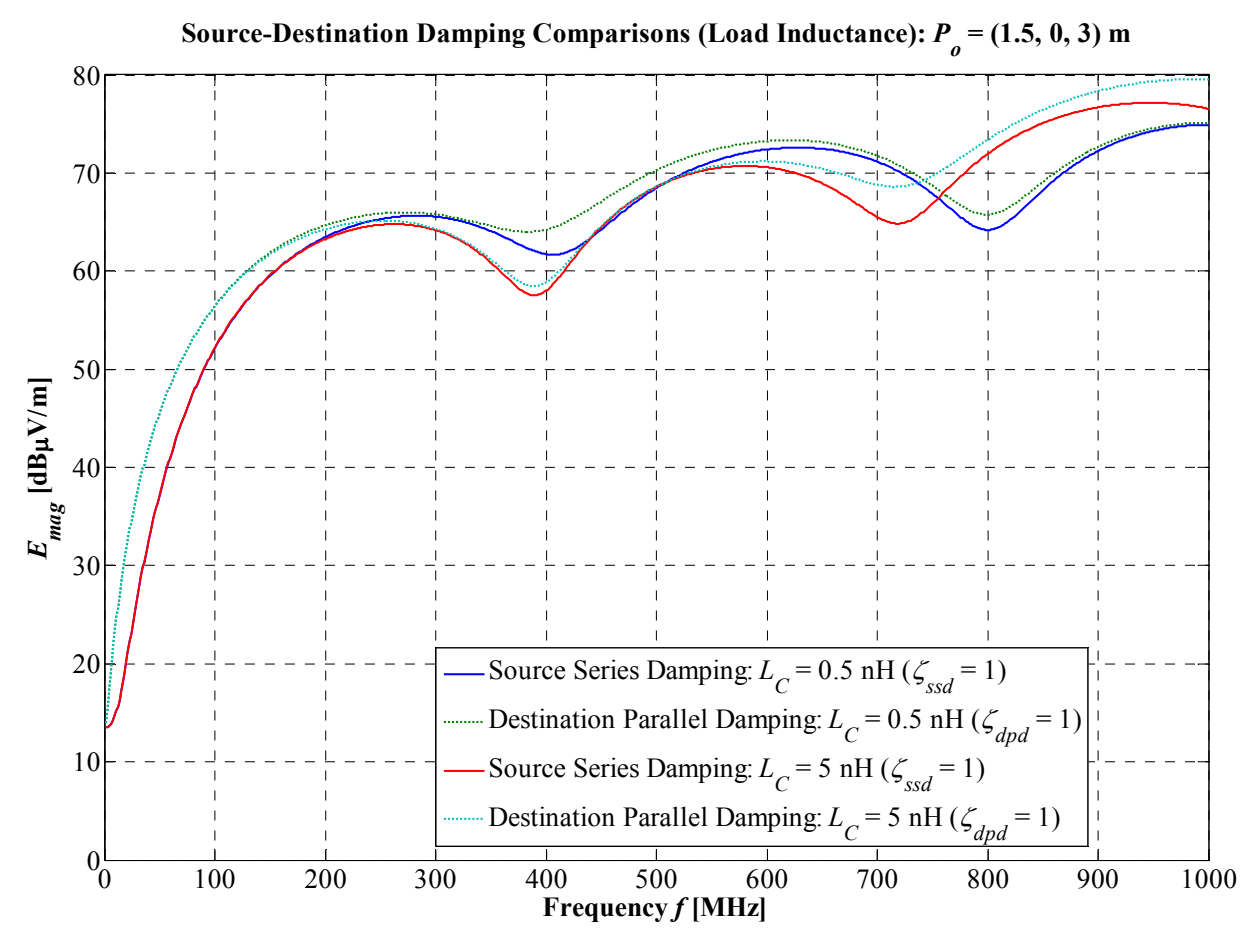

Figure 5.6 Effect of various load inductance at $P_{o}=(1.5,0,3) \mathrm{m}$ with

$$
Z_{o_{e f f}} \simeq 52.53 \Omega \text {. }
$$

\subsection{Conclusion}

From Figure 5.2, Figure 5.3 and Figure 5.4 it can be seen that source series damping is significantly beneficial in terms of radiated emission for very high damping values i.e. $\zeta=4$. However, it should be noted that such a high damping factor would not be used in practice in a digital system since signal integrity would be judged poor due to the long rise and fall time. One possible case where such a large damping factor may be used in practice is on an IO line where control of EMC is critical and a special regenerative receiver is used at the end of the line.

Factors other that EMC consideration studied in [7] shows that source series damping offers a number of advantages. A key contribution made in this thesis is the development of an efficient model that can be used to determine if there is any differentiation between source and destination damping as far as emissions are concerned. For the small number of cases considered in the thesis it would appear that there is no significant difference for practical damping factors. 


\section{Chapter 6. Conclusions and Future Work}

As can be seen from the plots, source series damping in general is slightly better than destination parallel damping. In particular for overdamped systems i.e. $\zeta_{s s d}=\zeta_{d p d}=4$, source series damping emissions are approximately $10 \mathrm{~dB}$ below destination parallel damping, for frequencies below $500 \mathrm{MHz}$. This damping is not realistic in practice as it would result in excessively long rise and fall times. However, only at this value of damping is there an observable difference. For realistic damping of one or less there is no apparent advantage in using source series damping.

Some topics of future work include:

1. The development of closed form expressions for the electric field. Further work according to $[1,6]$ has been undertaken which presents analytical expressions in a plane, which also include the effect of dielectric polarisation.

2. A general expression for a figure of merit relating to the frequency, geometry of the microstrip structure and damping terminations can be further investigated.

3. Extensions to the prediction of the radiated emissions from an infinite ground to a finite ground [31] and to improve the model with the inclusion of radiation loss may be an avenue for investigation. In terms of damping, a more accurate model can be developed to account for the load inductance and to extend from a second order damping model to higher order systems. 


\section{Appendix A. Results for Chapter 2}

\section{A.1 Transmission Line Wave Equations}

In the derivation of the Telegrapher's or wave equations, the T-type equivalent circuit model is used, with a symmetric structure, as shown Figure A.1. These derivations are based on lossless lines. The reason that the losses $R$ and $G$ can be incorporated is that they can be negligible compared to $L$ and $C$. The derivation begins with the application of Kirchoff's voltage and current laws to a differential length of a two-conductor TL modelled with a T-type section. By taking the limit as the differential length approaches zero the Telegrapher's equations are obtained.

To characterise a two-conductor TL consider a short segment of length $\Delta z$, directed along the $z$-axis, in the lead-up towards the derivation of the Telegrapher's equations.

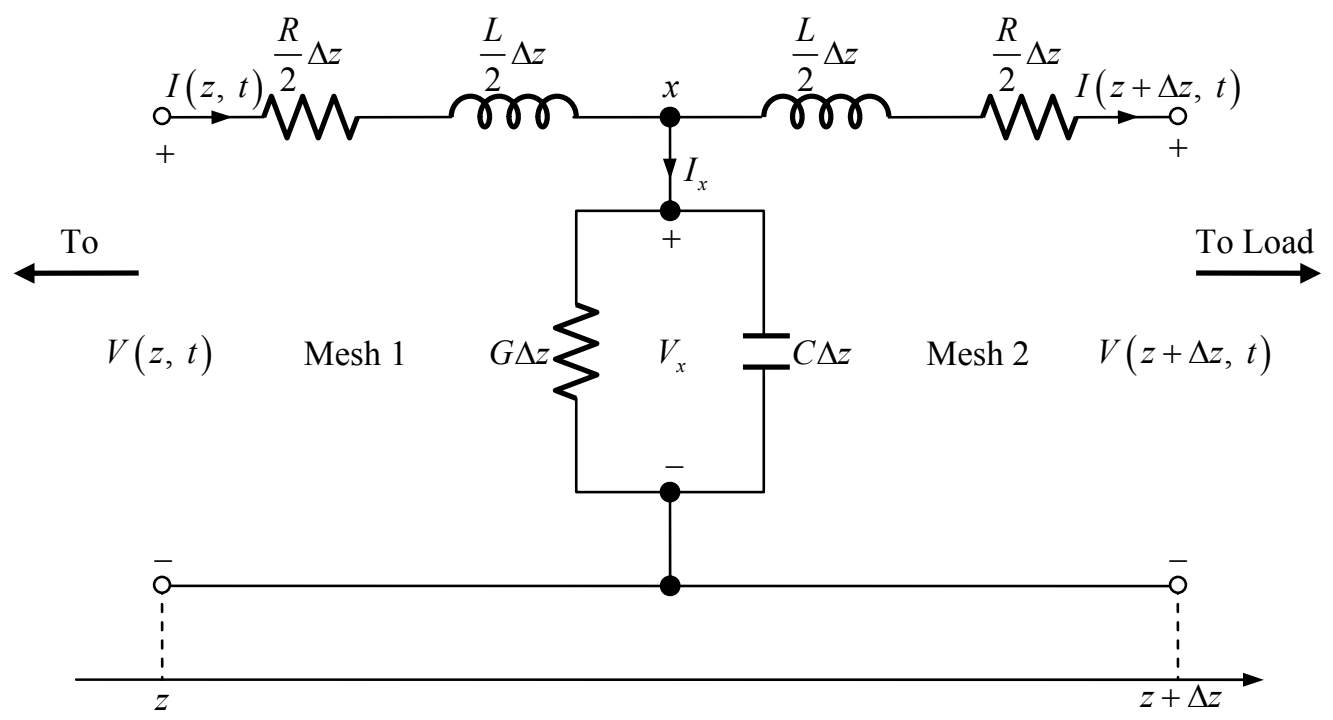

Figure A.1 T-type equivalent circuit model.

The Telegrapher's equations are a set of differential equations. In deriving these equations simple circuit analysis is required. By applying Kirchoff's voltage law around mesh 1

$$
V(z, t)=\frac{R}{2} \Delta z I(z, t)+\frac{L}{2} \Delta z \frac{\partial I(z, t)}{\partial t}+V_{x}
$$


and around mesh 2

$$
V_{x}=\frac{L}{2} \Delta z \frac{\partial I(z+\Delta z, t)}{\partial t}+\frac{R}{2} \Delta z I(z+\Delta z, t)+V(z+\Delta z, t)
$$

Substituting equation (A.2) into equation (A.1) and rearranging yields

$$
\begin{aligned}
-\frac{V(z+\Delta z, t)-V(z, t)}{\Delta z}= & \frac{R}{2}(I(z, t)+I(z+\Delta z, t)) \\
& +\frac{L}{2}\left(\frac{\partial I(z, t)}{\partial t}+\frac{\partial I(z+\Delta z, t)}{\partial t}\right)
\end{aligned}
$$

By taking the limit as $\Delta z \rightarrow 0$ equation (A.3) is expressed as

$$
\begin{aligned}
-\lim _{\Delta z \rightarrow 0} \frac{V(z+\Delta z, t)-V(z, t)}{\Delta z}= & \frac{R}{2} \lim _{\Delta z \rightarrow 0}(I(z, t)+I(z+\Delta z, t)) \\
& +\frac{L}{2} \lim _{\Delta z \rightarrow 0}\left(\frac{\partial I(z, t)}{\partial t}+\frac{\partial I(z+\Delta z, t)}{\partial t}\right),
\end{aligned}
$$

and by introducing the definition of partial derivatives, equation (A.4) becomes

$$
V(z, t)=R I(z, t)+L \frac{\partial I(z, t)}{\partial t}
$$

Equation (A.5) is the first of two foundational equations for obtaining the wave equations. The derivation of the second equation requires application of Kirchoff's current law at node $x$ resulting in

$$
I(z, t)=I(z+\Delta z, t)+G \Delta z V_{x}+C \Delta z \frac{\partial V_{x}}{\partial t} .
$$

By rearranging equation (A.6)

$$
-\frac{I(z+\Delta z, t)-I(z, t)}{\Delta z}=G V_{x}+C \frac{\partial V_{x}}{\partial t},
$$


and taking limits on both sides of equation (A.7), as $\Delta z \rightarrow 0$,

$$
-\lim _{\Delta z \rightarrow 0} \frac{I(z+\Delta z, t)-I(z, t)}{\Delta z}=G \lim _{\Delta z \rightarrow 0} V_{x}+C \lim _{\Delta z \rightarrow 0} \frac{\partial V_{x}}{\partial t} .
$$

By taking the limit on $V_{x}$ and as $\partial V_{x} / \partial t$ as $\Delta z \rightarrow 0$ result in

$$
\lim _{\Delta z \rightarrow 0} V_{x}=V(z, t),
$$

and

$$
\lim _{\Delta z \rightarrow 0} \frac{\partial V_{x}}{\partial t}=\frac{\partial V(z, t)}{\partial t}
$$

To obtain the second foundational equation substitute equations (A.9) and (A.10) into equation (A.8), yielding

$$
-\frac{\partial I(z, t)}{\partial z}=G V(z, t)+C \frac{\partial V(z, t)}{\partial t} .
$$

Assuming harmonic time dependence and complex sources, the current and voltage may be written as

$$
\begin{aligned}
& V(z, t)=\operatorname{Re}\left(\hat{V}(z) e^{j \omega t}\right), \\
& I(z, t)=\operatorname{Re}\left(\hat{I}(z) e^{j \omega t}\right),
\end{aligned}
$$

where $\hat{V}(z)$ and $\hat{I}(z)$ are phasors of $V(z, t)$ and $I(z, t)$ respectively.

Hence equation (A.5) can be expressed in terms of phasors

$$
-\frac{\partial \operatorname{Re}\left(\hat{V}(z) e^{j \omega t}\right)}{\partial z}=R \operatorname{Re}\left(\hat{I}(z) e^{j \omega t}\right)+L \frac{\operatorname{Re}\left(\hat{I}(z) e^{j \omega t}\right)}{\partial t}
$$


The linear operator $\operatorname{Re}($ ) operator may be removed and by applying the inverse Laplace transform (A.14) simplifies to

$$
-\frac{d \widehat{V}(z)}{d z}=(R+j \omega L) \hat{I}(z) .
$$

By using a similar approach equation (A.11) simplifies to

$$
-\frac{d \hat{I}(z)}{d z}=(G+j \omega C) \hat{V}(z) .
$$

Taking the second derivative of equation (A.15) with respect to $z$ and then rearranging yields

$$
-\frac{d \hat{I}(z)}{d z}=\frac{1}{R+j \omega L} \frac{d^{2} \hat{V}(z)}{d z^{2}} .
$$

Similarly, for equation (A.16)

$$
-\frac{d \hat{V}(z)}{d z}=\frac{1}{G+j \omega C} \frac{d^{2} \hat{I}(z)}{d z^{2}} .
$$

Substituting equation (A.17) into equation (A.16) and rearranging yields

$$
-\frac{d^{2} \widehat{V}(z)}{d z^{2}}=(R+j \omega L)(G+j \omega C) \widehat{V}(z) .
$$

By defining

$$
\gamma^{2}=(R+j \omega L)(G+j \omega C),
$$

and substituting into equation (A.19) yields one wave equation

$$
\frac{d^{2} \widehat{V}(z)}{d z^{2}}-\gamma^{2} \widehat{V}(z)=0
$$


Similarly by substituting equation (A.18) into equation (A.15), rearranging and simplifying yields the other wave equation

$$
\frac{d^{2} \hat{I}(z)}{d z^{2}}-\gamma^{2} \hat{I}(z)=0
$$

Equations (A.21) and (A.22) are the Telegrapher's equations.

\section{A.2 Voltage and Current along a Transmission Line}

To determine the voltage and current distributions along a TL, consider equations (A.21) and (A.22).

The general solution to the linear homogeneous differential equations can be expressed in terms of a forward travelling wave and a backward travelling wave expressed as

$$
\begin{gathered}
\hat{V}(z)=V_{\mathrm{o}}^{+} e^{-\gamma z}+V_{\mathrm{o}}^{-} e^{\gamma z}, \\
\hat{I}(z)=\frac{1}{Z_{\mathrm{o}}}\left(V_{\mathrm{o}}^{+} e^{-\gamma z}-V_{\mathrm{o}}^{-} e^{\gamma z}\right) .
\end{gathered}
$$

In (A.24) the characteristic impedance is introduced and is denoted by $Z_{\mathrm{o}}$.

To solve for the travelling voltages in equations (A.23) and (A.24) the boundary conditions at the ends of the TL are used.

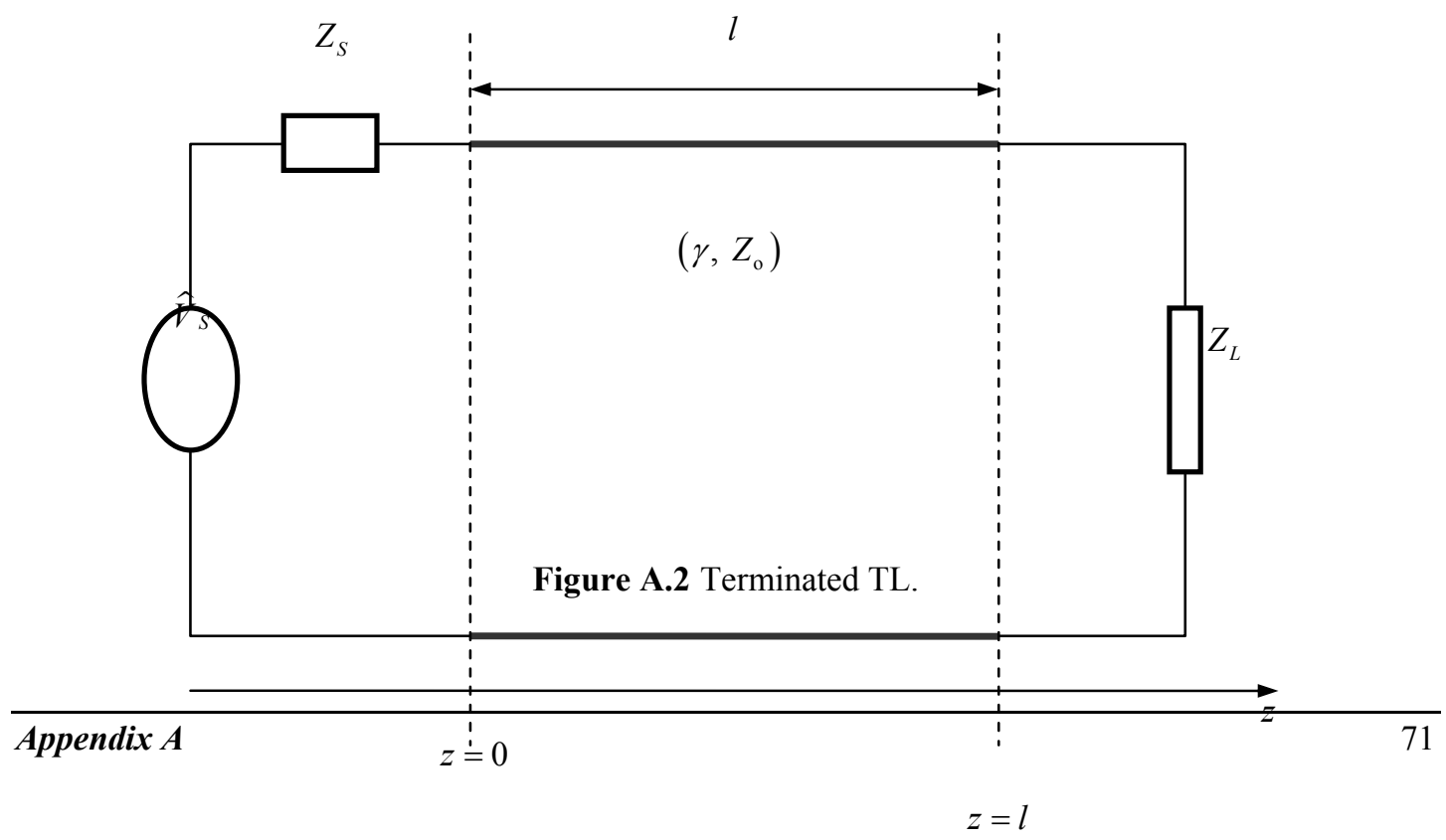


At boundary $z=0$

$$
\hat{V}(0)=\hat{V}_{S}-\hat{I}(0) Z_{S}
$$

Equation (A.23) evaluates to

$$
\widehat{V}(0)=V_{\mathrm{o}}^{+}+V_{\mathrm{o}}^{-}
$$

and equation (A.24) evaluates to

$$
\hat{I}(0)=\frac{1}{Z_{\mathrm{o}}}\left(V_{\mathrm{o}}^{+}-V_{\mathrm{o}}^{-}\right)
$$

Substituting equations (A.26) and (A.27) into equation (A.25) and rearranging yields

$$
\hat{V}_{S}=V_{\mathrm{o}}^{+}+V_{\mathrm{o}}^{-}+\frac{Z_{S}}{Z_{\mathrm{o}}}\left(V_{\mathrm{o}}^{+}-V_{\mathrm{o}}^{-}\right)
$$

At boundary $z=l$

$$
\hat{V}(l)=\hat{I}(l) Z_{L}
$$

Equation (A.23) evaluates to

$$
\widehat{V}(l)=V_{\mathrm{o}}^{+} e^{-\gamma l}+V_{\mathrm{o}}^{-} e^{\gamma l}
$$

and equation (A.24) evaluates to

$$
\hat{I}(l)=\frac{1}{Z_{\mathrm{o}}}\left(V_{\mathrm{o}}^{+} e^{-\gamma l}-V_{\mathrm{o}}^{-} e^{\gamma l}\right) .
$$

Substituting equations (A.30) and (A.31) into equation (A.29) and rearranging yields

$$
\frac{Z_{L}}{Z_{\mathrm{o}}}\left(V_{\mathrm{o}}^{+} e^{-\gamma l}-V_{\mathrm{o}}^{-} e^{\gamma l}\right)=V_{\mathrm{o}}^{+} e^{-\gamma l}+V_{\mathrm{o}}^{-} e^{\gamma l}
$$


By rearranging equation (A.28) and equation (A.32) they can be written as

$$
\begin{gathered}
V_{\mathrm{o}}^{+}-V_{\mathrm{o}}^{-} \frac{Z_{S}-Z_{\mathrm{o}}}{Z_{S}+Z_{\mathrm{o}}}=\hat{V}_{S} \frac{Z_{\mathrm{o}}}{Z_{S}+Z_{\mathrm{o}}}, \\
V_{\mathrm{o}}^{+} \frac{Z_{L}-Z_{\mathrm{o}}}{Z_{L}+Z_{\mathrm{o}}} e^{-2 \gamma l}-V_{\mathrm{o}}^{-}=0 .
\end{gathered}
$$

Observation from equations (A.33) and (A.34), lets define

$$
\begin{gathered}
\Gamma_{S}=\frac{Z_{S}-Z_{\mathrm{o}}}{Z_{S}+Z_{\mathrm{o}}}, \\
\Gamma_{L}=\frac{Z_{L}-Z_{\mathrm{o}}}{Z_{L}+Z_{\mathrm{o}}} .
\end{gathered}
$$

By using the above definitions, equations (A.33) becomes

$$
V_{\mathrm{o}}^{+}-V_{\mathrm{o}}^{-} \Gamma_{S}=\hat{V}_{S} \frac{Z_{\mathrm{o}}}{Z_{S}+Z_{\mathrm{o}}}
$$

Similarly equation (A.34) becomes

$$
V_{\mathrm{o}}^{+} \Gamma_{L} e^{-2 \gamma l}-V_{\mathrm{o}}^{-}=0
$$

Solving for equations (A.37) and (A.38) simultaneously, the amplitude of the forward travelling voltage is

$$
V_{\mathrm{o}}^{+}=\widehat{V}_{S} \frac{Z_{\mathrm{o}}}{Z_{S}+Z_{\mathrm{o}}} \frac{1}{1-\Gamma_{S} \Gamma_{L} e^{-2 \gamma l}},
$$

and the backward travelling voltage has amplitude of

$$
V_{\mathrm{o}}^{-}=\hat{V}_{S} \frac{Z_{\mathrm{o}}}{Z_{S}+Z_{\mathrm{o}}} \frac{\Gamma_{L} e^{-2 \gamma l}}{1-\Gamma_{S} \Gamma_{L} e^{-2 \gamma l}} .
$$


Substituting equations (A.39) and (A.40) into the general expressions given by equations (A.23) and (A.24), the voltage can be written as

$$
\widehat{V}(z)=\widehat{V}_{S} \frac{Z_{\mathrm{o}}}{Z_{S}+Z_{\mathrm{o}}} \frac{e^{-\gamma z}+\Gamma_{L} e^{-\gamma(2 l-z)}}{1-\Gamma_{S} \Gamma_{L} e^{-2 \gamma l}},
$$

and the current can be written as

$$
\hat{I}(z)=\hat{V}_{S} \frac{1}{Z_{S}+Z_{\mathrm{o}}} \frac{e^{-\gamma z}-\Gamma_{L} e^{-\gamma(2 l-z)}}{1-\Gamma_{S} \Gamma_{L} e^{-2 \gamma l}} .
$$

Expressed in hyperbolic form and using the identities

$$
\begin{aligned}
& \cosh x=\frac{1}{2}\left(e^{x}+e^{-x}\right), \\
& \sinh x=\frac{1}{2}\left(e^{x}-e^{-x}\right),
\end{aligned}
$$

the voltage can written as by defining $\widehat{V}(z)=\widehat{V}_{\text {lossy }}(z)$

$$
\hat{V}_{\text {lossy }}(z)=\frac{\hat{V}_{S} Z_{\mathrm{o}}}{D_{\text {lossy }}}\left(Z_{L} \cosh \gamma(l-z)+Z_{\mathrm{o}} \sinh \gamma(l-z)\right) .
$$

In a similar manner the current can be written as by defining $\hat{I}(z)=\hat{I}_{\text {lossy }}(z)$

$$
\hat{I}_{\text {lossy }}(z)=\frac{\hat{V}_{S}}{D_{\text {lossy }}}\left(Z_{\mathrm{o}} \cosh \gamma(l-z)+Z_{L} \sinh \gamma(l-z)\right),
$$

where

$$
D_{\text {lossy }}=Z_{\mathrm{o}}\left(Z_{S}+Z_{L}\right) \cosh \gamma l+\left(Z_{\mathrm{o}}^{2}+Z_{S} Z_{L}\right) \sinh \gamma l \text {. }
$$


In most cases a TL structure as represented in Figure A.2 is referenced at the origin so that the structure is symmetrical about $z=0$. Modifications can be made to equations (A.45) and (A.46) by introducing the following translation

$$
z^{\prime}=z+\frac{l}{2}
$$

where $z^{\prime}$ represent the new $z$ in equations (A.45) and (A.46).

By applying equation (A.48) to equations (A.45) and (A.46), now referenced at the origin, can be written as

$$
\begin{aligned}
& \hat{V}_{\text {lossy }}(z)=\frac{\hat{V}_{S} Z_{\mathrm{o}}}{D_{\text {lossy }}}\left(Z_{L} \cosh \gamma\left(\frac{l}{2}-z\right)+Z_{\mathrm{o}} \sinh \gamma\left(\frac{l}{2}-z\right)\right), \\
& \hat{I}_{\text {lossy }}(z)=\frac{\hat{V}_{S}}{D_{\text {lossy }}}\left(Z_{\mathrm{o}} \cosh \gamma\left(\frac{l}{2}-z\right)+Z_{L} \sinh \gamma\left(\frac{l}{2}-z\right)\right) .
\end{aligned}
$$

Equations (A.49) and (A.50) will be used for the forthcoming derivations.

\section{A.3 Voltage and Current along a Lossless Transmission Line}

The propagation constant can be defined as

$$
\gamma=\alpha+j \beta
$$

with $\alpha$ the attenuation constant in neper per metre, and $\beta$ is the wave number in radians per metre.

In the case of a lossless line the loss component $\alpha$ becomes zero hence

$$
\gamma=j \beta
$$


Using the identities

$$
\begin{aligned}
& \cosh j x=\cos x, \\
& \sinh j x=j \sin x,
\end{aligned}
$$

equations (A.45), (A.46) and (A.47) may then be written as (replacing the subscript with lossless):

$$
\begin{gathered}
\hat{V}_{\text {lossless }}(z)=\frac{\hat{V}_{S} Z_{\mathrm{o}}}{D_{\text {lossless }}}\left(Z_{L} \cos \beta\left(\frac{l}{2}-z\right)+j Z_{\mathrm{o}} \sin \beta\left(\frac{l}{2}-z\right)\right), \\
\hat{I}_{\text {lossless }}(z)=\frac{\hat{V}_{S}}{D_{\text {lossless }}}\left(Z_{\mathrm{o}} \cos \beta\left(\frac{l}{2}-z\right)+j Z_{L} \sin \beta\left(\frac{l}{2}-z\right)\right), \\
D_{\text {lossless }}=Z_{\mathrm{o}}\left(Z_{S}+Z_{L}\right) \cos \beta l+j\left(Z_{\mathrm{o}}^{2}+Z_{S} Z_{L}\right) \sin \beta l .
\end{gathered}
$$

The non-hyperbolic form of the first derivative of the voltage and current are

$$
\begin{aligned}
& \frac{d \hat{V}_{\text {lossless }}}{d z}(z)=\frac{\hat{V}_{S} Z_{\mathrm{o}} \beta}{D_{\text {lossless }}}\left(Z_{L} \sin \beta\left(\frac{l}{2}-z\right)-j Z_{\mathrm{o}} \cos \beta\left(\frac{l}{2}-z\right)\right), \\
& \frac{d \hat{I}_{\text {lossless }}(z)}{d z}=\frac{\hat{V}_{S} \beta}{D_{\text {lossless }}}\left(Z_{\mathrm{o}} \sin \beta\left(\frac{l}{2}-z\right)-j Z_{L} \cos \beta\left(\frac{l}{2}-z\right)\right),
\end{aligned}
$$

Where $D_{\text {lossless }}$ is the same as equation (A.57).

\section{A.3.1 Special Cases}

This section of the appendix presents some special cases of the voltage and current and their first derivatives for a lossless TL. Special cases include the short circuit and open circuit termination. Simplified equations at the source and load boundary ends are also given. 


\section{A.3.1.1 Short Circuit Load}

Substituting $Z_{L}=0$ into equations (A.55), (A.56), (A.58) and (A.59), yields

$$
\begin{aligned}
& \hat{V}_{\text {lossless }}(z)=j \frac{\widehat{V}_{S} Z_{\mathrm{o}}}{D_{\text {lossless }}} \sin \beta\left(\frac{l}{2}-z\right), \\
& \hat{I}_{\text {lossless }}(z)=\frac{\hat{V}_{S}}{D_{\text {lossless }}} \cos \beta\left(\frac{l}{2}-z\right) \text {, } \\
& \frac{d \hat{V}_{\text {lossless }}}{d z}(z)=-j \frac{\hat{V}_{S} \beta}{D_{\text {lossless }}} \cos \beta\left(\frac{l}{2}-z\right), \\
& \frac{d \hat{I}_{\text {lossless }}(z)}{d z}=\frac{\hat{V}_{S} \beta}{D_{\text {lossless }}} \sin \beta\left(\frac{l}{2}-z\right), \\
& D_{\text {lossless }}=Z_{S} \cos \beta l+j Z_{\mathrm{o}} \sin \beta l .
\end{aligned}
$$

\section{A.3.1.2 Open Circuit Load}

Taking the limit of equations (A.55), (A.56), (A.58) and (A.59) as $Z_{L} \rightarrow \infty$ yields

$$
\begin{aligned}
& \hat{V}_{\text {lossless }}(z)=\frac{\hat{V}_{S} Z_{\mathrm{o}}}{D_{\text {lossless }}} \cos \beta\left(\frac{l}{2}-z\right), \\
& \hat{I}_{\text {lossless }}(z)=j \frac{\hat{V}_{S}}{D_{\text {lossless }}} \sin \beta\left(\frac{l}{2}-z\right) \text {, } \\
& \frac{d \hat{V}_{\text {lossless }}}{d z}(z)=\frac{\hat{V}_{S} Z_{0} \beta}{D_{\text {lossless }}} \sin \beta\left(\frac{l}{2}-z\right), \\
& \frac{d \hat{I}_{\text {lossless }}(z)}{d z}=-j \frac{\hat{V}_{S} \beta}{D_{\text {lossless }}} \cos \beta\left(\frac{l}{2}-z\right), \\
& D_{\text {lossless }}=Z_{\mathrm{o}} \cos \beta l+j Z_{S} \sin \beta l .
\end{aligned}
$$




\section{A.3.1.3 Boundary Ends}

Here the boundary for the general, short and open circuit cases are derived by substituting $z=-l / 2$ and $z=l / 2$ into equations (A.65) - (A.68). The first is for the general case.

At boundary $z=-l / 2$

$$
\begin{aligned}
& \hat{V}_{\text {lossless }}\left(-\frac{l}{2}\right)=\frac{\hat{V}_{S} Z_{\mathrm{o}}}{D_{\text {lossless }}}\left(Z_{L} \cos \beta l+j Z_{\mathrm{o}} \sin \beta l\right), \\
& \hat{I}_{\text {lossless }}\left(-\frac{l}{2}\right)=\frac{\hat{V}_{S}}{D_{\text {lossless }}}\left(Z_{\mathrm{o}} \cos \beta l+j Z_{L} \sin \beta l\right), \\
& \frac{d \hat{V}_{\text {lossless }}\left(-\frac{l}{2}\right)=\frac{\hat{V}_{S} Z_{\mathrm{o}} \beta}{D_{\text {lossless }}}\left(Z_{L} \sin \beta l-j Z_{\mathrm{o}} \cos \beta l\right),}{d \hat{I}_{\text {lossless }}\left(-\frac{l}{2}\right)} \frac{\hat{V}_{S} \beta}{D_{\text {lossless }}}\left(Z_{\mathrm{o}} \sin \beta l-j Z_{L} \cos \beta l\right) .
\end{aligned}
$$

At boundary $z=l / 2$

$$
\begin{gathered}
\hat{V}_{\text {lossless }}\left(\frac{l}{2}\right)=\frac{\hat{V}_{S} Z_{0} Z_{L}}{D_{\text {lossless }}}, \\
\hat{I}_{\text {lossless }}\left(\frac{l}{2}\right)=\frac{\hat{V}_{S} Z_{\mathrm{o}}}{D_{\text {lossless }}}, \\
\frac{d \hat{V}_{\text {lossless }}\left(\frac{l}{2}\right)=-j \frac{\hat{V}_{S} Z_{\mathrm{o}}^{2} \beta}{D_{\text {lossless }}}}{d z} \\
\frac{d \hat{I}_{\text {lossless }}\left(\frac{l}{2}\right)}{d z}=-j \frac{\hat{V}_{S} Z_{L} \beta}{D_{\text {lossless }}} .
\end{gathered}
$$

$D_{\text {lossless }}$ is the same as equation (A.57). 


\section{A.3.1.3.1 Short Circuit Load}

At boundary $z=-l / 2$

$$
\begin{aligned}
& \widehat{V}_{\text {lossless }}\left(-\frac{l}{2}\right)=j \frac{\hat{V}_{S} Z_{\mathrm{o}}}{D_{\text {lossless }}} \sin \beta l, \\
& \hat{I}_{\text {lossless }}\left(-\frac{l}{2}\right)=\frac{\hat{V}_{S}}{D_{\text {lossless }}} \cos \beta l, \\
& \frac{d \hat{V}_{\text {lossless }}}{d z}\left(-\frac{l}{2}\right)=-j \frac{\hat{V}_{S} \beta}{D_{\text {lossless }}} \cos \beta l, \\
& \frac{d \hat{I}_{\text {lossless }}\left(-\frac{l}{2}\right)}{d z}=\frac{\hat{V}_{S} \beta}{D_{\text {lossless }}} \sin \beta l .
\end{aligned}
$$

At boundary $z=l / 2$

$$
\begin{aligned}
& \widehat{V}_{\text {lossless }}\left(\frac{l}{2}\right)=0 \text {, } \\
& \hat{I}_{\text {lossless }}\left(\frac{l}{2}\right)=\frac{\hat{V}_{S}}{D_{\text {lossless }}}, \\
& \frac{d \hat{V}_{\text {lossless }}}{d z}\left(\frac{l}{2}\right)=-j \frac{\hat{V}_{S} \beta}{D_{\text {lossless }}}, \\
& \frac{d \hat{I}_{\text {lossless }}\left(\frac{l}{2}\right)}{d z}=0 .
\end{aligned}
$$

$D_{\text {lossless }}$ is the same as equation (A.64). 


\section{A.3.1.3.2 Open Circuit Load}

At boundary $z=-l / 2$

$$
\begin{aligned}
& \widehat{V}_{\text {lossless }}\left(-\frac{l}{2}\right)=\frac{\widehat{V}_{S} Z_{\mathrm{o}}}{D_{\text {lossless }}} \cos \beta l, \\
& \hat{I}_{\text {lossless }}\left(-\frac{l}{2}\right)=j \frac{\hat{V}_{S}}{D_{\text {lossless }}} \sin \beta l, \\
& \frac{d \hat{V}_{\text {lossless }}}{d z}\left(-\frac{l}{2}\right)=\frac{\hat{V}_{s} Z_{\mathrm{o}} \beta}{D_{\text {lossless }}} \sin \beta l, \\
& \frac{d \hat{I}_{\text {lossless }}\left(-\frac{l}{2}\right)}{d z}=-j \frac{\hat{V}_{S} \beta}{D_{\text {lossless }}} \cos \beta l .
\end{aligned}
$$

At boundary $z=l / 2$

$$
\begin{gathered}
\hat{V}_{\text {lossless }}\left(\frac{l}{2}\right)=\frac{\hat{V}_{S} Z_{\mathrm{o}}}{D_{\text {lossless }}}, \\
\hat{I}_{\text {lossless }}\left(\frac{l}{2}\right)=0, \\
\frac{d \hat{V}_{\text {lossless }}\left(\frac{l}{2}\right)=0,}{d z}\left(\hat{I}_{\text {lossless }}\left(\frac{l}{2}\right)\right. \\
\frac{d z}{d z}=-j \frac{\hat{V}_{S} \beta}{D_{\text {lossless }}} .
\end{gathered}
$$

$D_{\text {lossless }}$ is the same as equation (A.69). 


\section{A.4 Decibel to Neper Conversion}

By definition the neper is used to express ratios such as gain or loss. The neper is similar to the decibel. Computing the ratio in neper uses the Naperian base $e \simeq 2.718281828 \ldots$.

The ratio of two voltage quantities $V_{1}$ and $V_{2}$ expressed in neper is

$$
\mathrm{Np}=\ln \left(\frac{A_{1}}{A_{2}}\right),
$$

or

$$
\frac{A_{1}}{A_{2}}=e^{\mathrm{Np}} .
$$

Writing the ratio of $V_{1}$ and $V_{2}$ in terms of decibel yield the following

$$
\mathrm{dB}=20 \log \left(\frac{A_{1}}{A_{2}}\right)
$$

Relating equation (A.96) to neper using equation (A.95) gives

$$
\mathrm{dB}=20 \log \left(e^{\mathrm{Np}}\right)
$$

Using the standard log and exponent identities

$$
\log _{y} x=\frac{\log x}{\log y}
$$

equation (A.97) in terms of decibels simplifies to

$$
\mathrm{Np}=\frac{\ln 10}{20} \mathrm{~dB}
$$

Equation (A.99) in terms of base 10 logarithms is written as

$$
\mathrm{Np}=\frac{1}{20 \log e} \mathrm{~dB} .
$$




\section{Appendix B. Results for Chapter 3}

\section{B.1 Schelkunoff's Equations in Compact Form}

To arrive at a compact representation of Schelkunoff's derivations, in cylindrical coordinates, for a straight thin conductor along the $z$-axis with sinusoidal current distribution consider [13]

$$
\begin{aligned}
& \widehat{E}_{z}=\frac{1}{4 \pi j \omega \varepsilon_{\mathrm{o}}}\left(\begin{array}{l}
\hat{I}^{\prime}\left(z_{1}\right) \frac{e^{-j \beta r_{1}(z)}}{r_{1}(z)}-\hat{I}^{\prime}\left(z_{2}\right) \frac{e^{-j \beta r_{2}(z)}}{r_{2}(z)} \\
+\hat{I}\left(z_{1}\right) \frac{\partial}{\partial z} \frac{e^{-j \beta r_{1}(z)}}{r_{1}(z)}-\hat{I}\left(z_{2}\right) \frac{\partial}{\partial z} \frac{e^{-j \beta r_{2}(z)}}{r_{2}(z)}
\end{array}\right), \\
& \widehat{E}_{\rho}=\frac{1}{4 \pi j \omega \varepsilon_{\mathrm{o}} \rho}\left(\begin{array}{l}
\hat{I}^{\prime}\left(z_{2}\right) e^{-j \beta r_{2}(z)} \cos \theta_{2}-\hat{I}^{\prime}\left(z_{1}\right) e^{-j \beta r_{1}(z)} \cos \theta_{1} \\
+\hat{I}\left(z_{1}\right)\left(j \beta \cos ^{2} \theta_{1}-\frac{\sin ^{2} \theta_{1}}{r_{1}(z)}\right) e^{-j \beta r_{1}(z)} \\
-\hat{I}\left(z_{2}\right)\left(j \beta \cos ^{2} \theta_{2}-\frac{\sin ^{2} \theta_{2}}{r_{2}(z)}\right) e^{-j \beta r_{2}(z)}
\end{array}\right), \\
& \widehat{E}_{\phi}=0 \text {. }
\end{aligned}
$$

The radial distance as a function of the $z$-component distance of observation point, $P_{o}$, from the $z$-coordinate of the end $z_{e}$ and a distance $\rho$ away, can be defined as

$$
r_{z_{e}}(z)=\sqrt{\rho^{2}+\left(z-z_{e}\right)^{2}}
$$

Observation of (B.1) requires the following evaluation by application of the quotient rule together with some simplification:

$$
\frac{\partial}{\partial z} \frac{e^{-j \beta r_{z_{e}}(z)}}{r_{z_{e}}(z)}=-\frac{e^{-j \beta r_{z_{e}}(z)}}{r_{z_{e}}^{2}(z)} \frac{\partial\left(r_{z_{e}}(z)\right)}{\partial z}\left(1+j \beta r_{z_{e}}(z)\right) .
$$


Evaluation of the partial differentiation in equation (B.5) then result in

$$
\frac{\partial\left(r_{z_{e}}(z)\right)}{\partial z}=\frac{z-z_{e}}{r_{z_{e}}(z)}
$$

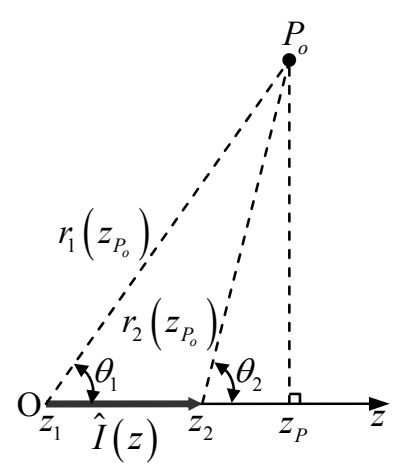

Figure B.1 Angular relationship of the radial function.

Further simplification can be made to equation (B.6) in terms of angles, where $r_{z_{e}}(z)$ defines the distance from the ends of the current source, $\hat{I}\left(z_{e}\right)$, to point $P_{o}$, such that

$$
\frac{\partial\left(r_{z_{e}}(z)\right)}{\partial z}=\cos \theta_{z_{e}}
$$

Substitution of equation (B.7) into equation (B.5) result in

$$
\frac{\partial}{\partial z} \frac{e^{-j \beta r_{z_{e}}(z)}}{r_{z_{e}}(z)}=-\frac{e^{-j \beta r_{z_{e}}(z)}}{r_{z_{e}}^{2}(z)}\left(1+j \beta r_{z_{e}}(z)\right) \cos \theta_{z_{e}} .
$$

Using the result from equation (B.8) and evaluating equation (B.1) yields the following

$$
\widehat{E}_{z}=\left.\left[j \frac{e^{-j \beta r_{z_{e}}\left(z_{P_{o}}\right)}}{4 \pi \omega \varepsilon_{\mathrm{o}}}\left(-\hat{I}^{\prime}\left(z_{e}\right) \frac{e^{-j \beta r_{z_{e}}\left(z_{P_{o}}\right)}}{r_{z_{e}}\left(z_{P_{o}}\right)}+\hat{I}\left(z_{e}\right) \frac{\left(1+j \beta r_{z_{e}}\left(z_{P_{o}}\right)\right)}{r_{z_{e}}^{2}\left(z_{P_{o}}\right)} \cos \theta_{z_{e}}\right)\right]\right|_{z_{e}=z_{2}} ^{z_{e}=z_{1}} .
$$


The compact form of equation (B.2) may be written as

$$
\widehat{E}_{\rho}=\left.\left[j \frac{e^{-j \beta r_{z_{e}}\left(z_{P_{o}}\right)}}{4 \pi \omega \varepsilon_{\mathrm{o}}}\left(\hat{I}^{\prime}\left(z_{e}\right) \frac{\cos \theta_{z_{e}}}{\rho}-\frac{\sin ^{2} \theta_{z_{e}}-j \beta r_{z_{e}}\left(z_{P_{o}}\right) \cos ^{2} \theta_{z_{e}}}{\rho r_{z_{e}}\left(z_{P_{o}}\right)} \hat{I}\left(z_{e}\right)\right)\right]\right|_{z_{e}=z_{2}} ^{z_{e}=z_{1}} .
$$

Defining the following equations

$$
\begin{aligned}
& K=\frac{1}{4 \pi \omega \varepsilon_{\mathrm{o}}}, \\
& \hat{\mathbf{I}}_{D}=\left[\begin{array}{l}
\hat{I}\left(z_{e}\right) \\
\hat{I}^{\prime}\left(z_{e}\right)
\end{array}\right], \\
& \widehat{\mathbf{E}}=\left[\begin{array}{c}
\widehat{E}_{z} \\
\widehat{E}_{\rho} \\
\widehat{E}_{\phi}
\end{array}\right], \\
& \mathbf{G}=e^{-j \beta r_{z_{e}}\left(z_{P_{o}}\right)}\left[\begin{array}{cc}
\frac{\left(1+j \beta r_{z_{e}}\left(z_{P_{o}}\right)\right) \cos \theta_{b}}{r_{z_{e}}^{2}\left(z_{P_{o}}\right)} & -\frac{1}{r_{z_{e}}\left(z_{P_{o}}\right)} \\
\frac{\sin ^{2} \theta_{z_{e}}-j \beta r_{z_{e}}\left(z_{P_{o}}\right) \cos ^{2} \theta z_{e}}{\rho r_{z_{e}}\left(z_{P_{o}}\right)} & \frac{\cos \theta_{z_{e}}}{\rho} \\
0 & 0
\end{array}\right],
\end{aligned}
$$

results in a compact matrix representation

$$
\widehat{\mathbf{E}}=\left.K\left[\mathbf{G} \hat{\mathbf{I}}_{D}\right]\right|_{z_{e}=z_{2}} ^{z_{e}=z_{1}}
$$




\section{B.2 Electric Field of a Hertzian Dipole Arbitrarily Placed in Free Space}

In many literature as in [15] the electric field for a Hertzian dipole carrying a current

$$
\hat{I}=\operatorname{Re}\left\{I_{\mathrm{O}} e^{j \omega t}\right\}
$$

in spherical coordinates may be written as

$$
\begin{gathered}
\widehat{E}_{r}=\frac{\eta \hat{I} d z}{2 \pi}\left(\frac{1}{r^{2}}-\frac{j}{\beta r^{3}}\right) e^{-j \beta r} \cos \theta, \\
\widehat{E}_{\theta}=\frac{\eta \hat{I} d z}{4 \pi}\left(\frac{j \beta}{r}+\frac{1}{r^{2}}-\frac{j}{\beta r^{3}}\right) e^{-j \beta r} \sin \theta, \\
\widehat{E}_{\phi}=0,
\end{gathered}
$$

centred at the origin along the $z$ direction.

Assuming that the Hertzian dipole is shifted to a different location with a different orientation as illustrated in Figure B.2.

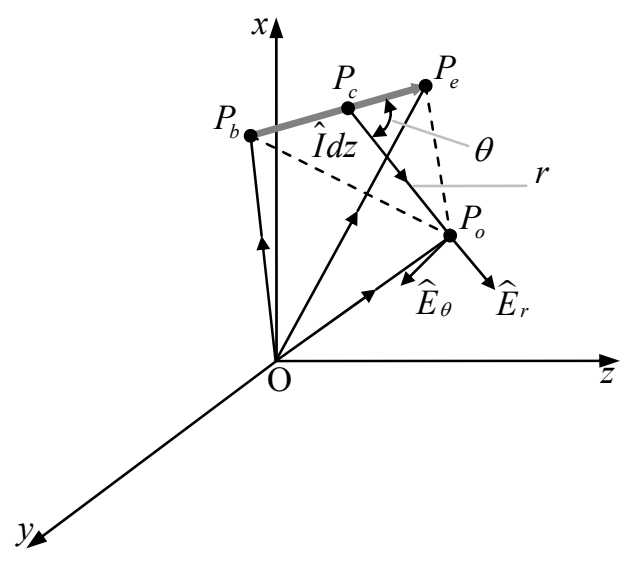

Figure B.2 An arbitrarily placed Hertzian dipole in free space.

From equations (B.17) and (B.18) the $r$ and $\theta$ variables are required as part in the determination of the electric field at an observation point. The $r$ variable is defined as the distance from the centre of the Hertzian dipole to the observation point. The $\theta$ 
variable is defined as the angle between the current vector along the $z$ direction and the vector from the $r$ distance vector. In Figure B.2 the Hertzian dipole can be said to have its coordinate system translated and rotated with respect to a reference coordinate system. Three points are defined along the current element as the beginning, centre and end points subscripted with $b, c$ and $e$ respectively. Only the beginning and end points are required to determine the variables in determining the electric field at the observation point with the $o$ subscript.

To determine the distance from $P_{c}$ to $P_{o}$ the vector from these two points must firstly be obtained. Begin with the vector from point $P_{b}$ to $P_{e}$ written as

$$
\mathbf{P}_{b e}=\mathbf{P}_{O e}-\mathbf{P}_{O b}
$$

The vector from $P_{b}$ to $P_{c}$ or from $P_{c}$ to $P_{e}$ becomes

$$
\mathbf{P}_{b c}=\mathbf{P}_{c e}=\frac{1}{2} \mathbf{P}_{b e}
$$

Using the result from equation (B.21) the position vector from $P_{c}$ is

$$
\mathbf{P}_{O c}=\mathbf{P}_{O b}+\frac{1}{2} \mathbf{P}_{b e}
$$

and in terms of the beginning and end position vectors equation (B.22) evaluates to

$$
\mathbf{P}_{O c}=\frac{1}{2}\left(\mathbf{P}_{O e}-\mathbf{P}_{O b}\right)
$$

From the observation position vector and equation (B.23)

$$
\mathbf{P}_{c o}=\mathbf{P}_{O o}-\mathbf{P}_{O c}=\mathbf{P}_{O o}-\frac{1}{2}\left(\mathbf{P}_{O e}-\mathbf{P}_{O b}\right)
$$

The distance $r$ in terms of Cartesian coordinates evaluates to

$$
r=\left|\mathbf{P}_{c o}\right|=\sqrt{x_{c o}^{2}+y_{c o}^{2}+z_{c o}^{2}} .
$$


The Hertzian dipole length $d z$ can also be evaluated in terms of the beginning and end position vectors in Cartesian coordinates as

$$
d z=\left|\mathbf{P}_{b e}\right|=\sqrt{\left(x_{O e}-x_{O b}\right)^{2}+\left(y_{O e}-y_{O b}\right)^{2}+\left(z_{O e}-z_{O b}\right)^{2}} .
$$

Now the only variable other variable left in calculating the electric field is to work out the angle, $\theta$, from the dot product notation. The angle between two vectors from [15] is given by

$$
\cos \theta_{A B}=\frac{\mathbf{A} \cdot \mathbf{B}}{|\mathbf{A}||\mathbf{B}|} .
$$

Hence

$$
\theta_{b e c o}=\cos ^{-1} \frac{\mathbf{P}_{b e} \cdot \mathbf{P}_{c o}}{\left|\mathbf{P}_{b e}\right|\left|\mathbf{P}_{c o}\right|}
$$

\section{B.2.1 Electric Field in Cartesian Coordinates}

Sometimes it is useful to have the electric field in Cartesian coordinates such as to be interpreted for further analysis.

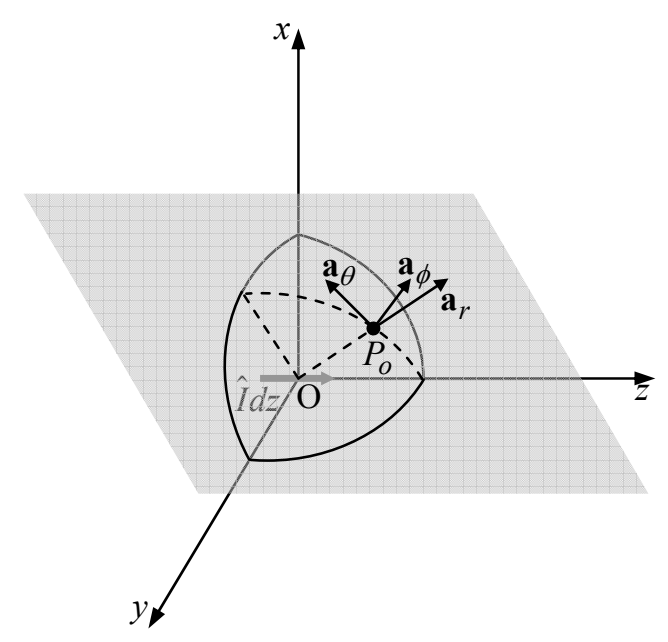

Figure B.3 Unit vectors at point $P_{o}$ in spherical coordinates. 
From Figure B.3 the cross product of $\mathbf{a}_{r}$ and $\mathbf{a}_{\theta}$ will result in $\mathbf{a}_{\phi}$. In other words any two vectors that lie on the shaded plane will result in an orthogonal vector. The cross vector product of two vectors $\mathbf{A}$ and $\mathbf{B}$ in Cartesian coordinates is given by [15]

$$
\mathbf{A} \times \mathbf{B}=\left(A_{y} B_{z}-A_{z} B_{y}\right) \mathbf{a}_{x}+\left(A_{z} B_{x}-A_{x} B_{z}\right) \mathbf{a}_{y}+\left(A_{x} B_{y}-A_{y} B_{x}\right) \mathbf{a}_{z} .
$$

In Figure B.2 the vector in the phi direction at $P_{o}$ with respect to the Hertzian dipole can be interpreted as

$$
\mathbf{P}_{b e} \times \mathbf{P}_{c o}=\left(P_{b e y} P_{c o z}-P_{b e z} P_{c o y}\right) \mathbf{a}_{x}+\left(P_{b e z} P_{c o x}-P_{b e x} P_{c o z}\right) \mathbf{a}_{y}+\left(P_{b e x} P_{c o y}-P_{b e y} P_{c o x}\right) \mathbf{a}_{z} .
$$

The unit phi vector then becomes

$$
\mathbf{a}_{\phi}=\frac{\mathbf{P}_{b e} \times \mathbf{P}_{c o}}{\left|\mathbf{P}_{b e} \times \mathbf{P}_{c o}\right|}
$$

with

$$
\left|\mathbf{P}_{b e} \times \mathbf{P}_{c o}\right|=\sqrt{\left(P_{b e y} P_{c o z}-P_{b e z} P_{c o y}\right)^{2}+\left(P_{b e z} P_{c o x}-P_{b e x} P_{c o z}\right)^{2}+\left(P_{b e x} P_{c o y}-P_{b e y} P_{c o x}\right)^{2}} .
$$

In using equation (B.29) the unit theta vector can be written as a triple vector cross product

$$
\mathbf{a}_{\theta}=\frac{\mathbf{P}_{b e} \times \mathbf{P}_{c o} \times \mathbf{P}_{c o}}{\left|\mathbf{P}_{b e} \times \mathbf{P}_{c o} \times \mathbf{P}_{c o}\right|}
$$

Also from equation (B.24) the unit radial vector becomes

$$
\mathbf{a}_{r}=\frac{\mathbf{P}_{c o}}{\left|\mathbf{P}_{c o}\right|}
$$

By using equations (B.31), (B.33) and (B.34) the electric field in Cartesian coordinates evaluates to

$$
\begin{aligned}
& \widehat{E}_{x}=a_{r x} \widehat{E}_{r}+a_{\theta x} \widehat{E}_{\theta}+a_{\phi x} \widehat{E}_{\phi} ; \\
& \widehat{E}_{y}=a_{r y} \widehat{E}_{r}+a_{\theta y} \widehat{E}_{\theta}+a_{\phi y} \widehat{E}_{\phi} ;
\end{aligned}
$$




$$
\widehat{E}_{z}=a_{r z} \widehat{E}_{r}+a_{\theta z} \widehat{E}_{\theta}+a_{\phi z} \widehat{E}_{\phi}
$$

whereby the scaling factor to the electric field components in spherical coordinates refers to the components of the unit vectors in equations (B.31), (B.33) and (B.34).

\section{B.2.2 Near-Zone Far-Zone Boundary}

Given the Hertzian dipole equation in spherical coordinates, electric fields that vary with $r^{-1}$, which is a far field term, lie in the far-zone; $r^{-2}$ is called the inductive term predictable from Biot-Savart's law and $r^{-3}$ is an electrostatic field term corresponding to the electric charges only. By taking the ratio of magnitudes from $r^{-2}$ and $r^{-1}$ terms as in equation (B.18), derivation of the near-zone to far-zone boundary can be obtained for a Hertzian dipole.

At observation point $P_{\mathrm{O}}$

$$
\begin{aligned}
\frac{\mid r_{\mathrm{O}}^{-2} \text { term } \mid}{\mid r_{\mathrm{O}}^{-1} \text { term } \mid} & =\frac{\frac{1}{r_{\mathrm{O}}^{2}}}{\frac{\beta}{r_{\mathrm{O}}}} . \\
& =\frac{\lambda}{2 \pi r_{\mathrm{O}}}
\end{aligned}
$$

This boundary is the transition between the induction and the radiation field term.

At the boundary

$$
\frac{\mid r_{\mathrm{O}}^{-2} \text { term } \mid}{\mid r_{\mathrm{O}}^{-1} \text { term } \mid}=1
$$

hence

$$
r_{\mathrm{O}}=\frac{\lambda}{2 \pi}
$$

It can be said that for $r$ less than $r_{\mathrm{O}}$, the terms $r^{-2}$ and $r^{-3}$ dominate. In the case of $r$ greater than $r_{\mathrm{O}}$, the term $r^{-1}$ is the most dominating. 


\section{Appendix C. Glossary}

This section is a quick reference guide to the many repeated acronyms, terminology and symbols used throughout this thesis. The purpose of these tables is to simplify the writing of new chapters, seeking to bring a clearer insight to the topics of discussion. Furthermore, some of these definitions are repeated to highlight their importance when encountered.

\section{C.1 Mathematical Conventions}

Variables are italic.

Vectors are bold and non-italic.

Variable constants may be non-italic subject to context.

Variable and vector subscripts or ${ }^{\text {superscripts }}$ may be non-italic subject to context.

Variables with a caret, $\hat{\text {, }}$ represent phasors that are time harmonic dependent denoted with $e^{j \omega t}$, and are suppressed for convenience.

\section{C.2 Abbreviated Units}

\begin{tabular}{|l|l|}
\hline Ampere & A \\
\hline Coulomb & C \\
\hline degree & ${ }^{\circ}$ or Deg \\
\hline Farad & F \\
\hline Henry & $\mathrm{H}$ \\
\hline Hertz & $\mathrm{Hz}$ \\
\hline Neper & $\mathrm{m}$ \\
\hline Neper & $\mathrm{Np}$ \\
\hline Ohm & $\Omega$ \\
\hline radian & $\mathrm{rad}$ \\
\hline Siemens & $\mathrm{S}=\Omega^{-1}=\widetilde{\sigma}$ \\
\hline
\end{tabular}




\begin{tabular}{|l|l|}
\hline time & $\mathrm{S}$ \\
\hline Volt & $\mathrm{V}$ \\
\hline Weber & $\mathrm{Wb}$ \\
\hline
\end{tabular}

\section{C.3 Table of Definitions}

\begin{tabular}{|l|l|l|}
\hline Conventional Symbol & Description & Units \\
\hline $\mathbf{A}$ & Magnetic vector potential & $\mathrm{Wb} / \mathrm{m}$ \\
\hline$C$ & Capacitance & $\mathrm{F}$ \\
\hline$d$ & Distance & $\mathrm{m}$ \\
\hline $\mathbf{E}$ & Electric field strength & $\mathrm{V} / \mathrm{m}$ \\
\hline$f$ & Frequency & $\mathrm{Hz}$ \\
\hline$H$ & Transfer function & - \\
\hline$I$ & Current & $\mathrm{A}$ \\
\hline $\mathbf{J}$ & Current density & $\mathrm{A} / \mathrm{m}^{2}$ \\
\hline$l$ & Line length & $\mathrm{m}$ \\
\hline$L$ & Inductance & $\mathrm{H}$ \\
\hline$Q$ & Charge & $\mathrm{C}$ \\
\hline$R$ & Resistance & $\Omega$ \\
\hline$S$ & Surface area & $\mathrm{m}$ \\
\hline$t$ & Time & $\mathrm{s}$ \\
\hline$u$ & Propagation velocity & $\mathrm{m} / \mathrm{s}$ \\
\hline$T$ & Period & $\mathrm{s}^{-1}$ \\
\hline$V$ & Potential & $\mathrm{V}$ \\
\hline$V_{S}$ & Source voltage & $\mathrm{V}$ \\
\hline$Z$ & Impedance & $\Omega$ \\
\hline$Z_{\mathrm{o}}$ & Characteristic impedance & $\Omega$ \\
\hline$Z_{L}$ & Load impedance & $\Omega$ \\
\hline$Z_{S}$ & Source impedance & \\
\hline & & $\Omega$ \\
\hline & & $\Omega$ \\
\hline & & \\
\hline & & \\
\hline & & \\
\hline
\end{tabular}




\begin{tabular}{|l|l|l|}
\hline Greek Symbol & Description & Units \\
\hline \hline$\alpha$ & Attenuation constant & $\mathrm{Np} / \mathrm{m}$ \\
\hline$\beta$ & Phase constant & $\mathrm{rad} / \mathrm{m}$ \\
\hline$\gamma$ & Complex propagation constant & $\mathrm{m}^{-1}$ \\
\hline$\delta$ & Skin depth & $\mathrm{m}$ \\
\hline$\varepsilon$ & Permittivity & $\mathrm{F} / \mathrm{m}$ \\
\hline$\eta$ & Intrinsic impedance & $\Omega$ \\
\hline$\lambda$ & Wavelength & $\mathrm{m}$ \\
\hline$\mu$ & Permeability & $\mathrm{H} / \mathrm{m}$ \\
\hline$\rho_{v}$ & Volume charge density & $\mathrm{C} / \mathrm{m}^{3}$ \\
\hline$\sigma$ & Conductivity & $\mathrm{S} / \mathrm{m}=\widetilde{\mathrm{rad} / \mathrm{m}}$ \\
\hline$\omega$ & Angular velocity & - \\
\hline$\zeta$ & Damping factor & - \\
\hline$\Gamma$ & Reflection coefficient & \\
\hline
\end{tabular}

\begin{tabular}{|l|l|l|}
\hline Coordinate Symbols & Description & Units \\
\hline \hline$O$ & Origin & - \\
\hline$P$ & Coordinate point & - \\
\hline$x$ & Cartesian coordinates & $\mathrm{m}$ \\
$y$ & & $\mathrm{~m}$ \\
$z$ & Cylindrical coordinates & $\mathrm{m}$ \\
\hline$\rho$ & & $\mathrm{m}$ \\
$\phi$ & & $\mathrm{rad}$ \\
$z$ & Spherical coordinates & $\mathrm{m}$ \\
\hline$r$ & & $\mathrm{~m}$ \\
$\theta$ & & $\mathrm{rad}$ \\
$\phi$ & & $\mathrm{rad}$ \\
\hline
\end{tabular}




\begin{tabular}{|l|l|}
\hline Mathematical Symbol & Description \\
\hline \hline a & Unit vector \\
\hline$D$ & Denominator \\
\hline $\mathbf{r}$ & Position vector \\
\hline$x$ & Unknown variable \\
\hline
\end{tabular}

\section{C.4 Physical Constants}

\begin{tabular}{|l|l|l|}
\hline$c_{\mathrm{o}}=2.99792458 \times 10^{8}$ & Speed of light in free space & $\mathrm{m} / \mathrm{s}$ \\
\hline$\varepsilon_{\mathrm{o}}=8.854187817 \times 10^{-12}$ & Permittivity in free space & $\mathrm{F} / \mathrm{m}$ \\
\hline$\mu_{\mathrm{o}}=1.256637061 \times 10^{-6}$ & Permeability in free space & $\mathrm{H} / \mathrm{m}$ \\
\hline$\varepsilon_{\mathrm{r}_{\mathrm{FR}-4}}=4.8$ & Relative permittivity of dielectric FR-4 & - \\
\hline$\sigma_{\mathrm{Cu}}=5.8 \times 10^{7}$ & Conductivity of copper & $\mathrm{S} / \mathrm{m}$ \\
\hline
\end{tabular}

\section{C.5 Acronyms}

\begin{tabular}{|l|l|}
\hline ACA & Australian Communications Authority \\
\hline DE & Differential Equation \\
\hline EEC & European Economic Community \\
\hline EMC & ElectroMagnetic Compatibility \\
\hline FCC & Federal Communications Commission \\
\hline FDTD & Finite-Difference Time-Domain \\
\hline FEM & Finite-Element Modelling \\
\hline IC & Intergrated Circuit \\
\hline IO & Input Output \\
\hline MoM & Method of Moments \\
\hline PCB & Printed Circuit Board \\
\hline PEEC & Partial Element Equivalent Circuit \\
\hline TEM & Transverse ElectroMagnetic \\
\hline TL & Transmission Line \\
\hline
\end{tabular}




\section{Appendix D. Chamber Map}

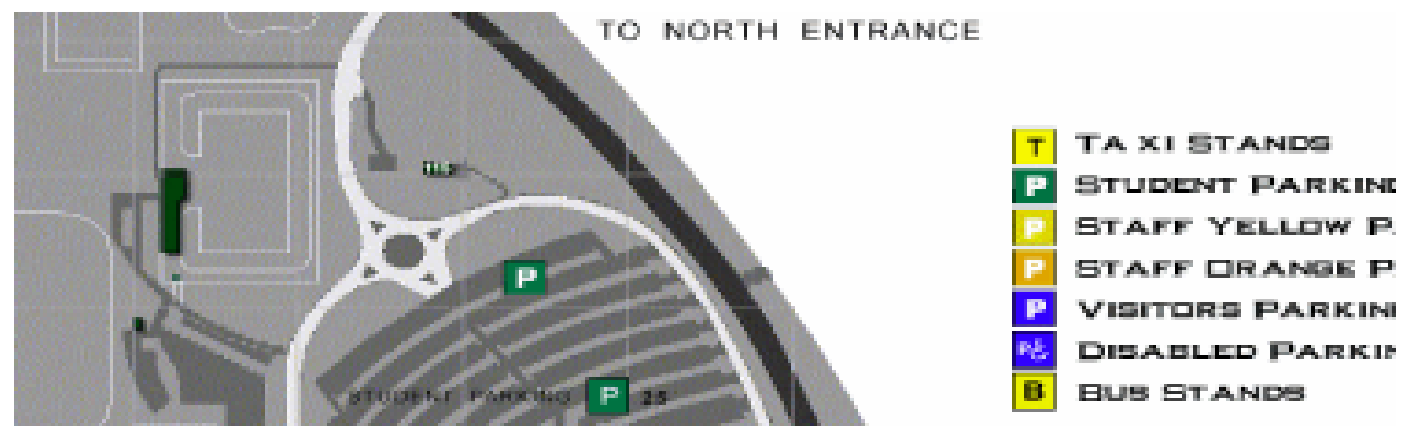

MC Chamber

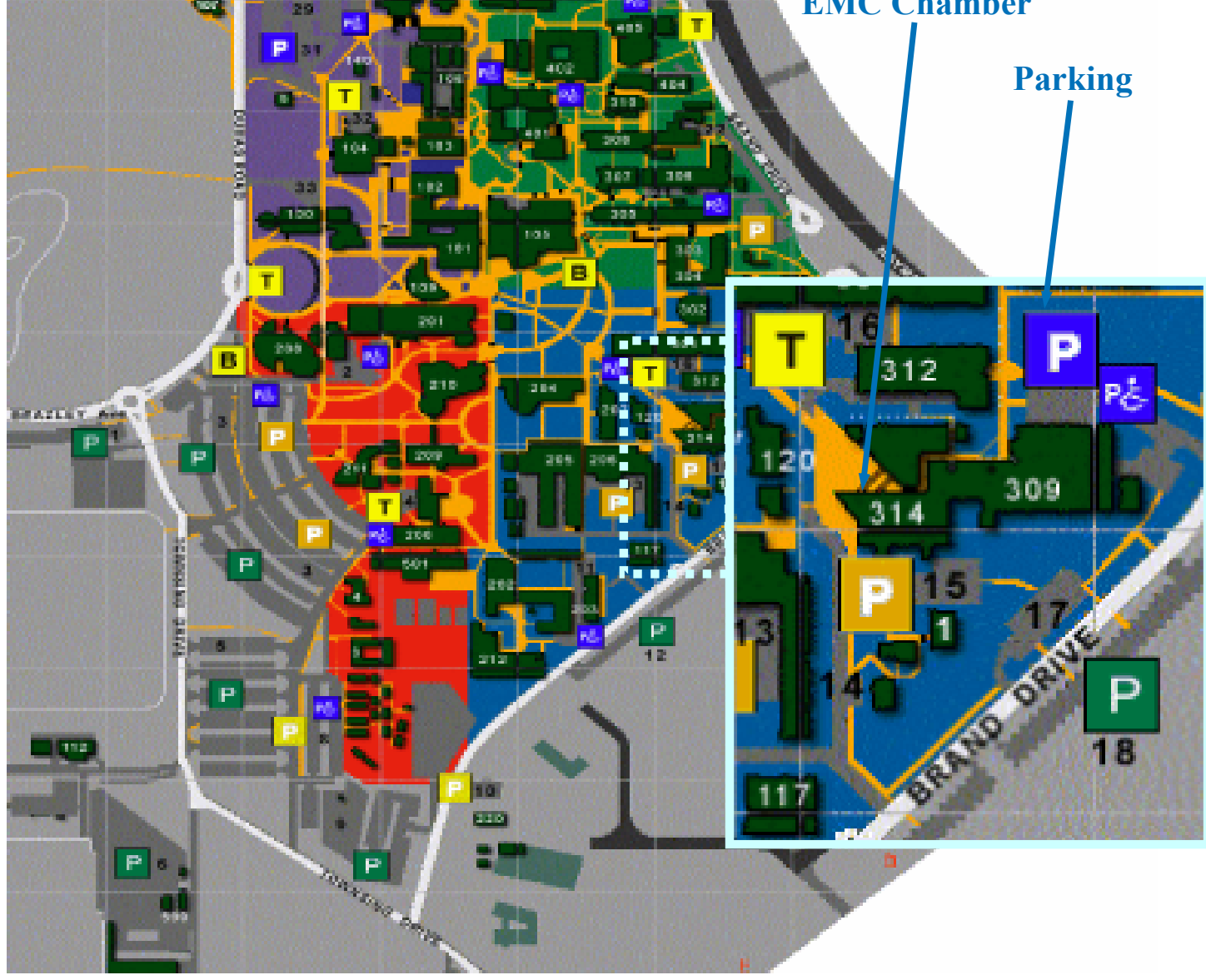

Figure F.1 Chamber location.

The EMC chamber is located at building 3.14 room 141. 


\section{References}

[1] Wong, B \& Trinkle, J 2006, 'Accurate measurements in semi-anechoic chambers', Electromagnetic Compatibility Symposium, Electromagnetic Compatibility Society of Australia, IEEE Electromagnetic Compatibility Society, pp. 11-20.

[2] Balanis, CA 1989 Advanced engineering electromagnetics, Wiley \& Sons, New York.

[3] Bokhari, SA 1998, 'Analysis of the effect of shunt traces on the radiation from printed circuit boards', IEEE International Symposium on EMC, pp. 621-623.

[4] Quak, D 2001, 'Analysis of transient radiation of a (traveling) current pulse on a straight wire segment', IEEE International Symposium on EMC, pp. 849-854.

[5] Paul, CR 1989, 'A comparison of the contributions of common-mode and differential-mode currents in radiated emissions', IEEE Transactions on $E M C$, vol. 31, no. 2, pp. 189-193.

[6] Trinkle, J \& Wong, B 2006, 'Derivation of analytic formulas for radiated far field in a plane from a microstrip transmission line', Draft, WATRI Internal Document, TM-EMC-068.

[7] Cantoni, A 1992, 'Digital interconnects', Draft, WATRI Internal Document, TM-EDL-064.

[8] Wong, B, Cantoni, A, Fynn, K \& Trinkle, J 2005, 'Effect of modelling fringing and losses for a microstrip on the radiated emission characteristics', IEEE International Symposium on EMC, pp. 557-562. 
[9] Leone, M \& Singer, H 1999, 'Efficient computation of radiated fields from finite-size printed circuit boards including the effect of dielectric layer', IEEE International Symposium on EMC, pp. 85-90.

[10] Bennett, WS 1996, 'The electromagnetic radiation of small circuits and small loop antennas', IEEE International Symposium on EMC, pp. 477-481.

[11] Yun, YH, Ham, SH \& Lee, YH 1998, 'The electromagnetic radiation with phase difference in rectangular loop antenna or rectangular loop circuit', IEEE International Symposium on EMC, pp. 381-386.

[12] Bennett, WS 1998, 'Electromagnetic radiations of small circuits and their effective control', IEEE International Symposium on EMC, pp. 319-324.

[13] Schelkunoff, SA 1943, Electromagnetic waves, Van Nostrand, New York.

[14] Schwarz, SE 1990, Electromagnetics for engineers, Philadelphia: Saunders College Pub, Philadelphia.

[15] Sadiku, MNO 1989, Elements of electromagnetics, Saunders College Pub., New York.

[16] Johnk, CTA 1988, Engineering electromagnetic fields and waves, Wiley \& Sons, New York.

[17] Daijavad, S, Janak, J, Heeb, H, Ruehli \& McBride, D 1990, 'A fast method for computing radiation from printed circuit boards', IEEE International Symposium on EMC, pp. 300-304.

[18] Upton, MEG \& Marvin, AC 1994, 'The fields due to a small loaded loop in free space', IEEE Transactions on EMC, vol. 36, no. 1, pp. 7-13.

[19] Canavero, FG 2001, 'Foreword: special issue on "recent advances in EMC of printed circuit boards"', IEEE Transactions on EMC, vol. 43, no. 4, pp. 414-415. 
[20] Moongilan, D 2001, 'Image and return current modeling of PCB traces for radiated emissions', IEEE International Symposium on EMC, pp. 927-932.

[21] Wong, B, Fynn, K, Schlagenhaufer, F \& Cantoni, A 2001, 'The impact of transmission line terminations on radiated emissions', IEEE International Symposium on EMC, vol. 1, pp. 52-56.

[22] Larrabee, DA 1998, 'Interaction of an electromagnetic wave with transmission lines, including reradiation', IEEE International Symposium on EMC, pp. 106-111.

[23] Paul, CR 1992, Introduction to electromagnetic compatibility, Microwave and optical engineering, Wiley \& Sons, New York.

[24] Radojicic, M \& Costache, G 1991, 'A model to predict radiated emissions from electronic circuits', IEEE 1991 International Symposium on EMC, pp. 54-57.

[25] Burke, GJ \& Poggio, AJ 1981, Numerical electromagnetics code (NEC) method of moments, Dept. of Energy, Washington, Springfield, Va.

[26] Costa, V, Caniggia, S \& Catello, L 1990, 'On the computation of electromagnetic field components from a transmission line: theory and application', IEEE International Symposium on EMC, pp. 651-656.

[27] Nucci, CA and Rachidi, F 1995, 'On the contribution of the electromagnetic field components in field-to-transmission line interaction', IEEE Transactions on EMC, vol. 37, no. 4, pp. 505-508.

[28] Archambeault, B 1999, 'Predicting EMC emissions from printed circuit boards using numerical modeling tools', 'International Symposium on EMC', pp. 361-364.

[29] Han, F \& Zhang, L, 'Radiation characteristics of transmission line in PCBs', IEEE International Symposium on EMC, pp. 153-156. 
[30] Brueck, SRJ 2000, 'Radiation from a dipole embedded in a dielectric slab', IEEE Journal on Selected Topics in Quantum Electronics, vol.6, no. 6, pp. 899-910.

[31] Simpson, JP, Goulette, RR \& Costache, GI 1988, 'Radiation from microstrip transmission lines', pp. 340-343.

[32] Bokhari, SA, Mosig, JR \& Gardiol, FE 1992, 'Radiation pattern computation of microstrip antennas on finite sized ground plane', IEE Proceedings- $H$, vol. 139, no. 3, pp. 278-286.

[33] Wong, B 2006, '2nd order damping approximations', Draft, WATRI Internal Document, TM-EDL-070.

[34] King, RWP 1955, Transmission-line theory, McGraw-Hill New York.

"Every reasonable effort has been made to acknowledge the owners of copyright material. I would be pleased to hear from any copyright owner who has been omitted or incorrectly acknowledged." 